\title{
Higgs branch localization in three dimensions
}

\author{
Francesco Benini ${ }^{a}$ and Wolfger Peelaers ${ }^{b}$ \\ ${ }^{a}$ Simons Center for Geometry and Physics, Stony Brook University, \\ Stony Brook, NY 11794, U.S.A. \\ ${ }^{b}$ C.N. Yang Institute for Theoretical Physics, Stony Brook University, \\ Stony Brook, NY 11794, U.S.A. \\ E-mail: fra.benini@gmail.com, wolfger.peelaers@stonybrook.edu
}

ABSTRACT: We show that the supersymmetric partition function of three-dimensional $\mathcal{N}=2$ R-symmetric Chern-Simons-matter theories on the squashed $S^{3}$ and on $S^{2} \times S^{1}$ can be computed with the so-called Higgs branch localization method, alternative to the more standard Coulomb branch localization. For theories that could be completely Higgsed by Fayet-Iliopoulos terms, the path integral is dominated by BPS vortex strings sitting at two circles in the geometry. In this way, the partition function directly takes the form of a sum, over a finite number of points on the classical Coulomb branch, of a vortex-string times an antivortex-string partition functions.

KEywords: Supersymmetric gauge theory, Solitons Monopoles and Instantons, ChernSimons Theories, Nonperturbative Effects

ArXiv EPrint: 1312.6078 


\section{Contents}

1 Introduction 2

2 Higgs branch localization on $S_{b}^{3} \quad 3$

2.1 Killing spinors on $S_{b}^{3} \quad 4$

2.2 The BPS equations $\quad 6$

2.3 BPS solutions: Coulomb, Higgs and vortices 9

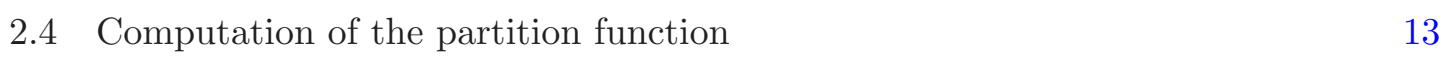

$\begin{array}{lll}\text { 2.4.1 One-loop determinants from an index theorem } & 13\end{array}$

$\begin{array}{lll}2.4 .2 & \text { Coulomb branch } & 14\end{array}$

$\begin{array}{ll}2.4 .3 & \text { Deformed Coulomb branch }\end{array}$

$\begin{array}{ll}2.4 .4 \text { Higgs branch and vortex partition function } & 16\end{array}$

$\begin{array}{ll}\text { 2.5 Matching with the Coulomb branch integral } & 18\end{array}$

2.6 Comparison with the two-dimensional vortex partition function 21

3 Higgs branch localization on $S^{2} \times S^{1} \quad 21$

3.1 Killing spinors on $S^{2} \times S^{1}$, supersymmetric index and deformed background 22

$\begin{array}{lll}3.2 & \text { The BPS equations } & 25\end{array}$

3.3 BPS solutions: Coulomb, Higgs and vortices 26

$\begin{array}{ll}3.4 & \text { Computation of the index } \\ & 29\end{array}$

3.4.1 One-loop determinants from the index theorem 29

$\begin{array}{ll}3.4 .2 \text { Coulomb branch } & 29\end{array}$

3.4.3 Deformed Coulomb branch 30

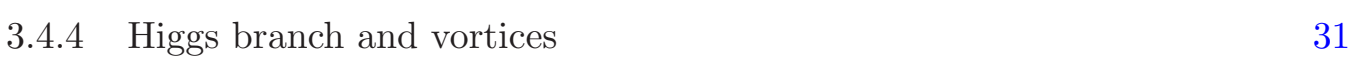

3.5 Matching with the Coulomb branch integral 33

4 Discussion $\quad 35$

$\begin{array}{ll}\text { A Spinor conventions } & 36\end{array}$

$\begin{array}{ll}\text { B Supersymmetric theories on three-manifolds } & 37\end{array}$

$\begin{array}{lll}\text { B.1 The superconformal algebra } & 37\end{array}$

B.2 Commuting Killing spinors $\quad 39$

$\begin{array}{lll}\text { B.3 Supersymmetric actions } & 39\end{array}$

C One-loop determinants from an index theorem 40 


\section{Introduction}

In the last few years there has been a huge development in the study of supersymmetric quantum field theories on compact manifolds, without topological twist. A stunning feature is that, in many cases, we are able to compute exactly the path integral and the expectation values of (local and non-local) operators that preserve some supersymmetry, with localization techniques $[1,2]$. The path integral can be reduced to something much simpler, like a matrix integral or a counting problem, and explicitly evaluated. After the seminal work of Pestun on $S^{4}$ [3], the techniques have been developed in many different contexts, essentially from two to five dimensions (see [3-22] for a non-exhaustive list).

Most of the work on supersymmetric theories with no twisting has been within the so-called Coulomb branch localization: the path integral is reduced to an ordinary integral over a "classical Coulomb branch", ${ }^{1}$ parametrized either by scalars in the vector multiplets, or by holonomies around circles. The integrand can contain non-perturbative contributions (e.g. if the geometry contains an $S^{4}$ or $S^{5}$ ), or not. For instance, in three dimensions $[9-13,15,16]$ the integrand is simply the one-loop determinant of all fields around the Coulomb branch configurations. It was observed by S. Pasquetti [23] (inspired by [24]), though, that the $S^{3}$ partition function can be rewritten as a sum over a finite set of points on the Coulomb branch, of the vortex times the antivortex partition functions [25], ${ }^{2}$ which do have a non-perturbative origin. In this paper we would like to gain a better understanding of this phenomenon, from the point of view of localization.

A mechanism responsible for such a "factorization" was first understood in $[4,5]$, in the analogous context of $2 \mathrm{~d} \mathcal{N}=(2,2)$ theories on $S^{2}$. It is possible to perform localization in an alternative way (that can be thought of either as adding a different deformation term, or as choosing a different path integration contour in complexified field space), dubbed Higgs branch localization, such that the BPS configurations contributing to the path integral are vortices at the north pole and antivortices at the south pole of $S^{2}$. Notice that such $2 \mathrm{~d}$ factorization for supersymmetric non-twisted theories is tightly related to the more general $t t^{*}$ setup [27].

In three dimensions quite some work has been done to understand factorization. Building on [24], the authors of [28] gave very general arguments why factorization should take place in terms of "holomorphic blocks". Factorization has been explicitly checked for $\mathrm{U}(N)$ theories with (anti)fundamentals on $S^{3}[29]$ and $S^{2} \times S^{1}[30,31]$, manipulating the Coulomb branch integrals. General continuous deformations of the geometry have been studied in $[14,32]$. Finally, the more general $t t^{*}$ setup has been developed in three and four dimensions [33]. Our approach is different.

In this paper we are after a Higgs branch localization mechanism in three-dimensional $\mathcal{N}=2$ R-symmetric Chern-Simons-matter theories, similar to the two-dimensional one [4]. We focus on the squashed sphere $S_{b}^{3}$ and on $S^{2} \times S^{1}$, knowing that more general backgrounds

\footnotetext{
${ }^{1}$ We used quotation marks because that would be the classical Coulomb branch on flat space, while the theories we consider are on compact Euclidean curved manifolds.

${ }^{2}$ The vortex partition function counts vortices in the $\Omega$-background on $\mathbb{R}^{2}$, in the same way as the instanton partition function of [26] counts instantons on $\mathbb{R}^{4}$.
} 
could be analyzed with the tools of $[32,34,35]$. We show that both on $S_{b}^{3}$ and $S^{2} \times S^{1}$, as in [4], an alternative localization (based on a different deformation term) is possible which directly yields an expression

$$
Z=\sum_{\text {vacua }} Z_{\mathrm{cl}} Z_{1 \text {-loop }}^{\prime} Z_{\mathrm{v}} Z_{\mathrm{av}}
$$

whenever the flat-space theory could be completely Higgsed by a Fayet-Iliopoulos term, and with some bounds on the Chern-Simons levels. The sum is over a finite set of points on the would-be "Coulomb branch", where some chiral multiplets get a VEV solving the D-term equations and completely Higgsing the gauge group. What is summed is a classical and oneloop contribution, evaluated on the vacua, times a vortex and an antivortex contributions, coming from BPS vortex-strings at the northern and southern circles of $S_{b}^{3}$ or $S^{2} \times S^{1}$. Both can be expressed in terms of the vortex partition function (VPF) on the twisted $\mathbb{R}_{\epsilon}^{2} \times S^{1}$ (a version of the VPF on the $\Omega$-deformed $\mathbb{R}^{2}[25]$ dressed by the KK modes on $S^{1}$, much like the $5 \mathrm{~d}$ instanton partition function of [36] on $\mathbb{R}_{\epsilon_{1}, \epsilon_{2}}^{4} \times S^{1}$ ). The precise identification of parameters depends on the geometry.

We expect the same method to work on other three-manifolds, for instance for the lens space index on $S_{b}^{3} / \mathbb{Z}_{p}$ [37, 38], and also in four dimensions on manifolds like $S^{3} \times S^{1}$ [39, 40], $S_{b}^{3} / \mathbb{Z}_{p} \times S^{1}[37,41]$ and $S^{2} \times T^{2}[18]$. We leave this for future work.

The paper is organized as follows. In section 2 we study the case of $S_{b}^{3}$ : we analyze the BPS equations and their solutions, we study the effect of the new deformation term responsible for Higgs branch localization, and write the general form of the partition function. We conclude with the example of a $\mathrm{U}(N)$ gauge theory with (anti)fundamentals [29]. In section 3 we do the same in the case of $S^{2} \times S^{1}$. We also consider the example of $\mathrm{U}(N)$ [31], and show that $S_{b}^{3}$ and $S^{2} \times S^{1}$ are controlled by the very same vortex partition function.

Note added. When this work was under completion, we became aware of [42] which has substantial overlap with our paper.

\section{$2 \quad$ Higgs branch localization on $S_{b}^{3}$}

We start by studying the path integral of three-dimensional $\mathcal{N}=2$ R-symmetric YangMills-Chern-Simons-matter theories on the squashed three-sphere $S_{b}^{3}$, where $b$ is a squashing parameter, and its supersymmetric localization. Such a path integral has been computed, with localization techniques, in [12], building on the works [9-11] (see also [13]). In their framework the path integral is dominated by BPS configurations that look like a classical Coulomb branch: the only non-vanishing field is an adjoint-valued real scalar in the vector multiplet (together with an auxiliary scalar), which can be diagonalized to the maximal torus. We thus dub this "Coulomb branch localization": the resulting expression in [12] is a matrix-model-like partition function, that we review in section 2.4.2.

Our goal is to perform localization in a different way, by including an extra $\mathcal{Q}$-exact term in the deformation action, ${ }^{3}$ so that the path integral is dominated by BPS configurations that look like vortex strings at a northern circle and antivortex strings at a southern

\footnotetext{
${ }^{3} \mathcal{Q}$ is a supercharge, and the path integral is not affected by the insertion of $\mathcal{Q}$-exact terms $[1,2]$.
} 
circle. Vortices exist on the Higgs branch, therefore we dub this Higgs branch localization, as in [4].

We will focus on a special class of backgrounds with three-sphere topology, the squashed three-sphere $S_{b}^{3}$ of [12] as we said, because our goal is to spell out how Higgs branch localization works. Much more general backgrounds are possible on $S^{3}[34,35]$, and we expect Higgs branch localization to be extendable to all those backgrounds easily. Moreover it has been shown in [32] that the supersymmetric partition function depends on the background through a single continuous parameter $b$ (there might be multiple connected components, though), therefore the computation on $S_{b}^{3}$ produces the full set of possible functions one can obtain in this way from the field theory.

\subsection{Killing spinors on $S_{b}^{3}$}

We consider a squashed three-sphere $S_{b}^{3}$ with metric [12]

$$
d s^{2}=f(\theta)^{2} d \theta^{2}+\tilde{\ell}^{2} \sin ^{2} \theta d \chi^{2}+\ell^{2} \cos ^{2} \theta d \varphi^{2},
$$

where $f(\theta)=\sqrt{\ell^{2} \sin ^{2} \theta+\tilde{\ell}^{2} \cos ^{2} \theta}$ and the squashing parameter $b$ is defined as $b=\sqrt{\tilde{\ell} / \ell}$. The ranges of coordinates are $\theta \in\left[0, \frac{\pi}{2}\right]$ and $\chi, \varphi \in[0,2 \pi)$. In fact, as apparent in [12] and remarked in [43] (see also [14]), any function $f(\theta)$ which asymptotes to $\tilde{\ell}, \ell$ at $\theta=0, \frac{\pi}{2}$ respectively and which gives a smooth metric, would lead to the same results. We choose the vielbein one-forms as

$$
e^{\underline{1}}=\ell \cos \theta d \varphi, \quad e^{\underline{2}}=-\tilde{\ell} \sin \theta d \chi, \quad e^{\underline{3}}=f(\theta) d \theta,
$$

yielding the non-zero components of the spin connection $\omega^{\underline{13}}=-\frac{\ell}{f} \sin \theta d \varphi$ and $\omega^{\underline{23}}=$ $-\frac{\tilde{\ell}}{f} \cos \theta d \chi$. We underline the flat coordinates in this frame. We also turn on a background gauge field that couples to the $\mathrm{U}(1)_{R}$ R-symmetry current:

$$
V=\frac{1}{2}\left(1-\frac{\ell}{f}\right) d \varphi+\frac{1}{2}\left(1-\frac{\tilde{\ell}}{f}\right) d \chi
$$

The twisted Killing spinor equation ${ }^{4} D_{\mu} \epsilon=\gamma_{\mu} \hat{\epsilon}$ (where $\gamma_{\underline{a}}$ are Pauli matrices) is then solved by the two spinors [12]

$$
\epsilon=\frac{1}{\sqrt{2}}\left(\begin{array}{c}
e^{-\frac{i}{2}(\varphi+\chi-\theta)} \\
-e^{-\frac{i}{2}(\varphi+\chi+\theta)}
\end{array}\right), \quad \bar{\epsilon}=\frac{1}{\sqrt{2}}\left(\begin{array}{c}
e^{\frac{i}{2}(\varphi+\chi+\theta)} \\
e^{\frac{i}{2}(\varphi+\chi-\theta)}
\end{array}\right)
$$

by assigning R-charges $R[\epsilon]=-1$ and $R[\bar{\epsilon}]=1$. In fact they satisfy

$$
D_{\mu} \epsilon=\frac{i}{2 f} \gamma_{\mu} \epsilon, \quad D_{\mu} \bar{\epsilon}=\frac{i}{2 f} \gamma_{\mu} \bar{\epsilon} .
$$

We also define the charge conjugate spinor $\tilde{\epsilon} \equiv-\bar{\epsilon}^{c}=i \epsilon$. For spinor conventions see appendix A.

\footnotetext{
${ }^{4}$ In our conventions $D_{\mu}=\partial_{\mu}+\frac{1}{4} \omega_{\mu}^{a b} \gamma_{a b}-i V_{\mu}$. Charge conjugation is $\epsilon^{c}=C \epsilon^{*}=\gamma_{2} \epsilon^{*}$, having chosen $C=\gamma_{\underline{2}}$.
} 
Two bilinears that we will need are:

$$
\xi^{\underline{a}}=i \bar{\epsilon} \gamma^{\underline{a}} \epsilon=-\epsilon^{\dagger} \gamma^{\underline{\underline{a}}} \epsilon=(-i \cos \theta, i \sin \theta, 0), \quad \bar{\epsilon} \epsilon=i \epsilon^{\dagger} \epsilon=i
$$

Using the coordinate frame $(\varphi, \chi, \theta)$ we have

$$
\xi^{\mu}=i \bar{\epsilon} \gamma^{\mu} \epsilon=\left(\frac{1}{\ell}, \frac{1}{\tilde{\ell}}, 0\right) .
$$

There are also two useful scalar bilinears, $\rho$ and $\alpha$ defined in (B.9), which take values $\rho=0$ and $\alpha=-\frac{1}{f}-\xi^{\mu} V_{\mu}=-\frac{1}{2}\left(\frac{1}{\ell}+\frac{1}{\ell}\right)$. Therefore the commutator of SUSY transformations (B.6) is

$$
\left[\delta_{\epsilon}, \delta_{\bar{\epsilon}}\right]=\mathcal{L}_{\xi}^{A}-\sigma-\frac{i}{2}\left(\frac{1}{\ell}+\frac{1}{\tilde{\ell}}\right) R
$$

It will be useful to perform a frame rotation such that the Killing vector field $\xi=\xi^{\mu} \partial_{\mu}$ becomes one of the frame vectors. We then define the non-underlined frame and its dual basis of vectors:

$$
\begin{array}{lll}
e^{1}=-f(\theta) d \theta & e^{2}=\cos \theta \sin \theta(\ell d \varphi-\tilde{\ell} d \chi) & e^{3}=\ell \cos ^{2} \theta d \varphi+\tilde{\ell} \sin ^{2} \theta d \chi \\
e_{1}=-f(\theta)^{-1} \partial_{\theta} & e_{2}=\ell^{-1} \tan \theta \partial_{\varphi}-\tilde{\ell}^{-1} \cot \theta \partial_{\chi} & e_{3}=\ell^{-1} \partial_{\varphi}+\tilde{\ell}^{-1} \partial_{\chi} .
\end{array}
$$

In particular $\xi=e_{3}$. In this basis the spin connection reads

$$
\omega^{a b}=\left(\begin{array}{ccc}
0 & -\frac{\ell}{f} \sin ^{2} \theta d \varphi-\frac{\tilde{\ell}}{f} \cos ^{2} \theta d \chi & \frac{\sin 2 \theta}{2 f}(-\ell d \varphi+\tilde{\ell} d \chi) \\
\frac{\ell}{f} \sin ^{2} \theta d \varphi+\frac{\tilde{\ell}}{f} \cos ^{2} \theta d \chi & 0 & -d \theta \\
\frac{\sin 2 \theta}{2 f}(\ell d \varphi-\tilde{\ell} d \chi) & d \theta & 0
\end{array}\right)
$$

and the Killing spinors become

$$
\epsilon=\left(\begin{array}{c}
0 \\
-e^{-\frac{i}{2}(\varphi+\chi)}
\end{array}\right), \quad \bar{\epsilon}=\left(\begin{array}{c}
e^{\frac{i}{2}(\varphi+\chi)} \\
0
\end{array}\right)
$$

as well as $\tilde{\epsilon}=-\bar{\epsilon}^{c}=i \epsilon$. The relation between the two bases is $e^{a}=\left(\begin{array}{ccc}0 & 0 & -1 \\ \sin \theta & \cos \theta & 0 \\ \cos \theta & -\sin \theta & 0\end{array}\right)_{a \underline{a}} e^{\underline{a}}$, where the matrix has determinant one. In the rest of this section we will use the nonunderlined frame.

To conclude let us describe the metric of the squashed three-sphere using Hopf coordinates $\phi_{H}=\varphi-\chi$ and $\psi_{H}=\varphi+\chi$, in which the Killing vector $\xi=\left(\frac{1}{\ell}+\frac{1}{\hat{\ell}}\right) \partial_{\psi_{H}}+\left(\frac{1}{\ell}-\frac{1}{\hat{\ell}}\right) \partial_{\phi_{H}}$. On the round sphere of radius $1 \xi=2 \partial_{\psi_{H}}$ generates pure motion around the Hopf fiber, whilst the squashing introduces an additional rotation of the base space $S^{2}$ with fixed points at $\theta=0$ and $\theta=\frac{\pi}{2}$. The metric (2.1) reads in these coordinates:

$$
\begin{aligned}
d s^{2}= & f(\theta)^{2} d \theta^{2}+\frac{\ell^{2} \tilde{\ell}^{2} \sin ^{2} 2 \theta}{4\left(\ell^{2} \cos ^{2} \theta+\tilde{\ell}^{2} \sin ^{2} \theta\right)} d \phi_{H}^{2} \\
& +\frac{1}{4}\left(\ell^{2} \cos ^{2} \theta+\tilde{\ell}^{2} \sin ^{2} \theta\right)\left(d \psi_{H}+\frac{\ell^{2} \cos ^{2} \theta-\tilde{\ell}^{2} \sin ^{2} \theta}{\ell^{2} \cos ^{2} \theta+\tilde{\ell}^{2} \sin ^{2} \theta} d \phi_{H}\right)^{2} .
\end{aligned}
$$

In fact one could instead take $\partial_{\phi_{H}}$ as the Hopf vector field, and rewrite the metric in the same form as above but with $\psi_{H} \leftrightarrow \phi_{H}$. 


\subsection{The BPS equations}

We will now consider the BPS equations for vector and chiral multiplets, and how they can be obtained as the zero-locus of the bosonic part of a $\mathcal{Q}$-exact deformation action. See appendix B for the SUSY transformations.

First we define

$$
W^{r}=\frac{1}{2} \varepsilon^{r m n} F_{m n}, \quad F_{m n}=\varepsilon_{m n r} W^{r},
$$

so that $\frac{1}{2} F_{m n} F^{m n}=W_{m} W^{m}$. Then, from (B.14), the BPS equations for the vector multiplet are

$$
\begin{aligned}
& 0=\mathcal{Q} \lambda=i\left(W_{\mu}+D_{\mu} \sigma\right) \gamma^{\mu} \epsilon-\left(D+\frac{\sigma}{f}\right) \epsilon \\
& 0=\mathcal{Q} \lambda^{\dagger}=-i \tilde{\epsilon}^{\dagger} \gamma^{\mu}\left(W_{\mu}-D_{\mu} \sigma\right)+\tilde{\epsilon}^{\dagger}\left(D+\frac{\sigma}{f}\right) .
\end{aligned}
$$

Recall that in Euclidean signature we regard $\lambda$ and $\lambda^{\dagger}$ as independent fields. It is convenient to use the non-underlined frame and the Killing spinors in (2.11); after taking sums and differences of the components, we get the BPS equations:

$$
0=W_{1}-i D_{2} \sigma, \quad 0=W_{2}+i D_{1} \sigma, \quad 0=W_{3}-i\left(D+\frac{\sigma}{f}\right), \quad 0=D_{3} \sigma .
$$

In fact - as it is standard - the equations (2.15) can be derived as the zero-locus of the bosonic part of a $\mathcal{Q}$-exact deformation action, whose Lagrangian is

$$
\mathcal{L}_{\mathrm{YM}}^{\mathrm{def}}=\mathcal{Q} \operatorname{Tr}\left[\frac{(\mathcal{Q} \lambda)^{\ddagger} \lambda+\lambda^{\dagger}\left(\mathcal{Q} \lambda^{\dagger}\right)^{\ddagger}}{4}\right] .
$$

Here the action of the formal adjoint operator $\ddagger$ on $\mathcal{Q} \lambda$ and $\mathcal{Q} \lambda^{\dagger}$ is:

$$
\begin{aligned}
& (\mathcal{Q} \lambda)^{\ddagger}=\epsilon^{\dagger}\left[-i \gamma^{\mu}\left(W_{\mu}+D_{\mu} \sigma^{\dagger}\right)-\left(D+\frac{\sigma^{\dagger}}{f}\right)\right] \\
& \left(\mathcal{Q} \lambda^{\dagger}\right)^{\ddagger}=\left[i\left(W_{\mu}-D_{\mu} \sigma^{\dagger}\right) \gamma^{\mu}+\left(D+\frac{\sigma^{\dagger}}{f}\right)\right] \tilde{\epsilon},
\end{aligned}
$$

where we treat $\sigma$ as a complex field. The operator $\ddagger$ reduces to $\dagger$ when $A_{\mu}$ and $D$ are taken real. Decomposing $\sigma=\sigma_{R}+i \sigma_{I}$ into its real and imaginary parts, we find that the bosonic part of $\mathcal{L}_{\mathrm{YM}}^{\text {def }}$ is a positive sum of squares:

$$
\begin{gathered}
\frac{1}{4} \operatorname{Tr}\left[(\mathcal{Q} \lambda)^{\ddagger} \mathcal{Q} \lambda+\mathcal{Q} \lambda^{\dagger}\left(\mathcal{Q} \lambda^{\dagger}\right)^{\ddagger}\right]=\operatorname{Tr}\left\{\frac{1}{2}\left(W_{1}+D_{2} \sigma_{I}\right)^{2}+\frac{1}{2}\left(W_{2}-D_{1} \sigma_{I}\right)^{2}\right. \\
\left.+\frac{1}{2}\left(W_{3}+\frac{\sigma_{I}}{f}\right)^{2}+\frac{1}{2}\left(D_{3} \sigma_{I}\right)^{2}+\frac{1}{2} \sum_{a=1,2,3}\left(D_{a} \sigma_{R}\right)^{2}+\frac{1}{2}\left(D+\frac{\sigma_{R}}{f}\right)^{2}\right\} .
\end{gathered}
$$

If we restrict to real fields, $\sigma_{I}=0$, from the zero locus of this action we recover the localization locus $F_{\mu \nu}=0$ and $\sigma=-f D=$ const, as in [9]. On a three-sphere, $F_{\mu \nu}=0$ allows us to set $A_{\mu}=0$, then $D_{\mu} \sigma=\partial_{\mu} \sigma$ and finally $\sigma$ can be diagonalized. On the other hand the equations (2.15) allow for more general solutions with complex $\sigma$. 
As in [4], Higgs branch localization can be achieved by adding another $\mathcal{Q}$-exact term to the deformation action. Consider

$$
\mathcal{L}_{\mathrm{H}}^{\mathrm{def}}=\mathcal{Q} \operatorname{Tr}\left[\frac{i\left(\epsilon^{\dagger} \lambda-\lambda^{\dagger} \tilde{\epsilon}\right) H(\phi)}{2}\right]
$$

whose bosonic part is

$$
\left.\mathcal{L}_{\mathrm{H}}^{\mathrm{def}}\right|_{\text {bos }}=\operatorname{Tr}\left[\left(W_{3}-i\left(D+\frac{\sigma}{f}\right)\right) H(\phi)\right] .
$$

The action $S_{\mathrm{H}}^{\mathrm{def}}=\int \mathcal{L}_{\mathrm{H}}^{\text {def }}$ is both $\mathcal{Q}$-exact and $\mathcal{Q}$-closed. ${ }^{5} H(\phi)$ is a generic real function of the complex scalar fields $\phi, \phi^{\dagger}$ in chiral multiplets, ${ }^{6}$ taking values in the adjoint representation. Actually one could even consider more general functions $H(\phi, \sigma)$-and we mention the interesting fact that $H(\phi, \sigma)=H(\phi)+\kappa \sigma_{I}$ would lead to Yang-Mills-Chern-Simons vortex equations - but we will not do so in this paper.

The bosonic part of the new deformation term $\mathcal{L}_{\mathrm{H}}^{\text {def }}$ is not positive definite. However if we consider the sum $\mathcal{L}_{\mathrm{YM}}^{\text {def }}+\mathcal{L}_{\mathrm{H}}^{\text {def }}$, the auxiliary field $D$ appears quadratically without derivatives and can be integrated out exactly by performing the Gaussian path integral. This corresponds to imposing

$$
D+\frac{\sigma_{R}}{f}=i H(\phi)
$$

in other words $D+\sigma_{R} / f$ is formally taken out of the real contour. The bosonic part of what we are left with is a positive sum of squares:

$$
\begin{aligned}
\mathcal{L}_{\mathrm{YM}}^{\text {def }}+\left.\mathcal{L}_{\mathrm{H}}^{\text {def }}\right|_{D, \text { bos }}= & \operatorname{Tr}\left[\frac{1}{2}\left(W_{1}+D_{2} \sigma_{I}\right)^{2}+\frac{1}{2}\left(W_{2}-D_{1} \sigma_{I}\right)^{2}\right. \\
& \left.+\frac{1}{2}\left(W_{3}+\frac{\sigma_{I}}{f}+H(\phi)\right)^{2}+\frac{1}{2}\left(D_{3} \sigma_{I}\right)^{2}+\frac{1}{2} \sum_{a=1,2,3}\left(D_{a} \sigma_{R}\right)^{2}\right] .
\end{aligned}
$$

The BPS equations describing its zero-locus are then

$$
0=W_{1}+D_{2} \sigma_{I}, \quad 0=W_{2}-D_{1} \sigma_{I}, \quad 0=W_{3}+\frac{\sigma_{I}}{f}+H(\phi), \quad 0=D_{3} \sigma_{I}, \quad 0=D_{a} \sigma_{R} .
$$

These equations differ from (2.15) only by the fact that the "D-term equation" (2.21) has been imposed.

Let us now consider the chiral multiplets, transforming in some (possibly reducible) representation of the gauge and flavor symmetry group. At this point it is useful to introduce some notation. We call $\mathfrak{R}$ the (possibly reducible) representation of the gauge

\footnotetext{
${ }^{5}$ While exactness is manifest in (2.19), closeness follows from an argument in [20]. If $\epsilon^{\dagger}, \tilde{\epsilon}$ were fields, the integral of the trace in (2.19) would be invariant under $\mathcal{Q}^{2}$ because it is a neutral scalar. Therefore if $\epsilon$ is invariant under the bosonic operator $\mathcal{Q}^{2}$, then $S_{\mathrm{H}}^{\mathrm{def}}$ is $\mathcal{Q}$-closed. It is easy to check that $\mathcal{Q}^{2} \epsilon=$ $\mathcal{L}_{\xi} \epsilon+\frac{i}{2}\left(\ell^{-1}+\tilde{\ell}^{-1}\right) \epsilon=0$.

${ }^{6}$ If we want to be sure that $\mathcal{L}_{\mathrm{H}}^{\text {def }}$ does not change the vacuum structure of the theory, we should limit ourselves to functions $H$ that do not modify the behavior of the action at infinity in field space [44]. This is the case if $H(\phi)$ is quadratic.
} 
and flavor symmetry group under which all chiral multiplets transform. Accordingly, we consider a vector multiplet for the full gauge and flavor symmetry, the components for the gauge group being dynamical and those for the flavor group being external, and whose real scalar we call $\mathfrak{S}$. On a supersymmetric background, external vector multiplets should satisfy the same BPS equations (2.14), but of course they do not have a kinetic action. Real expectation values of the external components of $\mathfrak{S}$ are the so-called real masses, so coupling a chiral multiplet in representation $\mathfrak{R}$ to $\mathfrak{S}$ includes real masses as well. ${ }^{7}$ On the other hand, we decompose $\mathfrak{R}$ into irreducible representations of the gauge group: $\mathfrak{R}=\bigoplus_{i} \mathcal{R}_{i}$. In this notation, each chiral multiplet in representation $\mathcal{R}_{i}$ couples to $\sigma$ and to its real mass term $m_{i}$. The projection of $\mathfrak{S}$ on the representation $\mathcal{R}_{i}$ is $\left.\mathfrak{S}\right|_{\mathcal{R}_{i}}=\sigma+m_{i}$.

For each irreducible gauge representation $\mathcal{R}$, the BPS equations $\mathcal{Q} \psi=\mathcal{Q} \psi^{\dagger}=0$ give

$$
\begin{array}{ll}
0=D_{3} \phi-\left(\sigma+m+i \frac{q}{f}\right) \phi & 0=e^{-\frac{i}{2}(\chi+\varphi)}\left(D_{1}-i D_{2}\right) \phi+i e^{\frac{i}{2}(\chi+\varphi)} F \\
0=D_{3} \phi^{\dagger}+\phi^{\dagger}\left(\sigma+m+i \frac{q}{f}\right) & 0=e^{\frac{i}{2}(\chi+\varphi)}\left(D_{1}+i D_{2}\right) \phi^{\dagger}+i e^{-\frac{i}{2}(\chi+\varphi)} F^{\dagger}
\end{array}
$$

where $m$ is the mass and $q$ is the R-charge (all fields in $\mathcal{R}$ must have the same mass and R-charge). Imposing the reality conditions $\phi=\left(\phi^{\dagger}\right)^{\dagger}, F=\left(F^{\dagger}\right)^{\dagger}$ and decomposing $\sigma$ into real and imaginary parts as before, the equations simplify to

$$
\left(\sigma_{R}+m\right) \phi=0, \quad D_{3} \phi-i\left(\sigma_{I}+\frac{q}{f}\right) \phi=0, \quad\left(D_{1}-i D_{2}\right) \phi=0, \quad F=0 .
$$

In passing we note that, since $\xi=e_{3}$ and using the first equation $\left.\mathbb{R e} \mathfrak{S}\right|_{\mathcal{R}_{i}} \phi=0$, the second one is

$$
0=\xi^{\mu}\left(\partial_{\mu}-i A_{\mu}-i q V_{\mu}\right) \phi-i\left(\sigma_{I}+\frac{q}{f}\right) \phi=\left[\mathcal{L}_{\xi}^{A}-\frac{i q}{2}\left(\frac{1}{\ell}+\frac{1}{\tilde{\ell}}\right)-\left.\mathfrak{S}\right|_{\mathcal{R}_{i}}\right] \phi=\mathcal{Q}^{2} \phi
$$

As before, these equations can also be obtained from the canonical deformation action

$$
\mathcal{L}_{\text {mat }}^{\text {def }}=\mathcal{Q} \frac{(\mathcal{Q} \psi)^{\dagger} \psi+\psi^{\dagger}\left(\mathcal{Q} \psi^{\dagger}\right)^{\dagger}}{4}
$$

Up to total derivatives, its bosonic part reads

$$
\left.\mathcal{L}_{\text {mat }}^{\text {def }}\right|_{\text {bos }}=\frac{1}{2}\left|D_{3} \phi-i\left(\sigma_{I}+\frac{q}{f}\right) \phi\right|^{2}+\frac{1}{2}\left|\left(D_{1}-i D_{2}\right) \phi\right|^{2}+\frac{1}{2}\left|\left(\sigma_{R}+m\right) \phi\right|^{2}+\frac{1}{2}|F|^{2},
$$

where we recognize once again the BPS equations.

\footnotetext{
${ }^{7}$ In our discussion we are not completely general. In three dimensions, the flavor symmetry group usually includes topological (or magnetic) symmetries which do not act on the microscopic chiral multiplets in the Lagrangian, but rather on monopole operators, and real mass parameters can be included for those symmetries as well. For instance, a $\mathrm{U}(1)$ gauge theory has a $\mathrm{U}(1)_{T}$ topological symmetry and a real mass for it is the Fayet-Iliopoulos term. However in our formalism FI terms have to be included by hand, rather than turning on the corresponding component of $\mathfrak{S}$.
} 
To conclude this section, let us rewrite the BPS equations in components since it will be useful later on. For the vector multiplet we find

$$
\begin{array}{rlrl}
\ell^{-1} \tilde{\ell}^{-1} F_{\varphi \chi} & =\left(-\ell^{-1} \sin ^{2} \theta D_{\varphi}+\tilde{\ell}^{-1} \cos ^{2} \theta D_{\chi}\right) \sigma_{I} \\
\ell^{-1} F_{\theta \varphi}+\tilde{\ell}^{-1} F_{\theta \chi} & =-D_{\theta} \sigma_{I} & & =\left(\ell^{-1} D_{\varphi}+\tilde{\ell}^{-1} D_{\chi}\right) \sigma_{I} \\
\ell^{-1} \tan \theta F_{\theta \varphi}-\tilde{\ell}^{-1} \cot \theta F_{\theta \chi} & =f(\theta) H(\phi)+\sigma_{I} & & =D_{\mu} \sigma_{R},
\end{array}
$$

and for the chiral multiplet we get $0=\left(\sigma_{R}+m\right) \phi=F$ as well as

$$
\begin{aligned}
\left(\ell^{-1} D_{\varphi}+\tilde{\ell}^{-1} D_{\chi}\right) \phi & =i\left(\sigma_{I}+\frac{q}{f}\right) \phi \\
\left(f(\theta)^{-1} D_{\theta}+i \ell^{-1} \tan \theta D_{\varphi}-i \tilde{\ell}^{-1} \cot \theta D_{\chi}\right) \phi & =0 .
\end{aligned}
$$

\subsection{BPS solutions: Coulomb, Higgs and vortices}

We will now analyze the solutions to (2.15), (2.23) and (2.25). First, let us recall the solutions for the standard choice $H(\phi)=0$.

Coulomb-like solutions. Consider (2.15) and (2.25). We solve them along a "real" contour where $A_{\mu}, \sigma, D$ are real, in particular $\sigma_{I}=0$, and $\left(\phi, \phi^{\dagger}\right),\left(F, F^{\dagger}\right)$ are conjugate pairs. Moreover we assume that all chiral multiplets have positive R-charge. As mentioned before, the solutions are [9]

$$
A_{\mu}=0, \quad \sigma=-f D=\text { const }, \quad \phi=F=0 .
$$

Let us check that there are no solutions with non-trivial $\phi$. We can Fourier expand along the compact directions $\varphi, \chi$ :

$$
\phi(\theta, \varphi, \chi)=\sum_{m, n \in \mathbb{Z}} c_{m n}(\theta) e^{i n \varphi} e^{i m \chi} .
$$

The first equation in (2.30) imposes the constraint $q=2(m \ell+n \tilde{\ell}) /(\ell+\tilde{\ell})$ for $m, n \in \mathbb{Z}$. In particular for incommensurable values of $\ell, \tilde{\ell}$, either $q$ is one of the special values above and in this case there is only one Fourier mode $(m, n)$, or $\phi=0$ is the only solution. Assuming that $\ell, \tilde{\ell}$ are incommensurable and that $m, n$ are fixed and solve the constraint, the second equation in (2.30) reduces to $\left(\sin 2 \theta \partial_{\theta}+q \cos 2 \theta+L f(\theta)\right) \phi=0$ with $L=2(m-n) /(\ell+\tilde{\ell})$. The solution is

$$
\phi(\theta, \varphi, \chi)=\left(\frac{1-s(\theta)}{1+s(\theta)}\right)^{\frac{L \ell}{4}}\left(\frac{1-\tilde{s}(\theta)}{1+\tilde{s}(\theta)}\right)^{-\frac{L \tilde{\ell}}{4}}(\sin 2 \theta)^{-q / 2} e^{i n \varphi} e^{i m \chi}
$$

with

$$
s(\theta)=\sqrt{\frac{\ell^{2}+\tilde{\ell}^{2}-\left(\ell^{2}-\tilde{\ell}^{2}\right) \cos 2 \theta}{2 \ell^{2}}}, \quad \tilde{s}(\theta)=\sqrt{\frac{\ell^{2}+\tilde{\ell}^{2}-\left(\ell^{2}-\tilde{\ell}^{2}\right) \cos 2 \theta}{2 \tilde{\ell}^{2}}} .
$$


The functions $s, \tilde{s}$ are monotonic and positive, with $s(0)=\tilde{s}\left(\frac{\pi}{2}\right)^{-1}=\tilde{\ell} / \ell$ and $s\left(\frac{\pi}{2}\right)=\tilde{s}(0)=$ 1. For $q>0$ there are no smooth solutions. For $q=0$ (then $m=n=0$ ) there is the constant Higgs-like solution $\phi=\phi_{0}$ that we will re-encounter below (in this case, $\sigma_{R}$ is constrained by $\left.\left(\sigma_{R}+m\right) \phi=0\right)$, but we will not consider it here since we assumed that R-charges are positive.

Now let us study the new solutions with non-trivial $H(\phi)$. We integrate $D$ out first, i.e. we solve (2.23) and (2.25) and impose a "real" contour for all fields but $D$ (in particular $\sigma_{I}=0$ again). We also take vanishing R-charges, $q=0$ : arbitrary R-charges can be recovered by analytic continuation of the final result in the real masses, as in [4]. We make the following choice for $H(\phi)$ :

$$
H(\phi)=\zeta-\sum_{i, a} T_{\text {adj }}^{a} \phi_{i}^{\dagger} T_{\mathcal{R}_{i}}^{a} \phi_{i}
$$

where the sum is over the representations $\mathcal{R}_{i}$ and the gauge symmetry generators $T^{a}$ in representation $\mathcal{R}_{i}$. The adjoint-valued parameter $\zeta$ is defined as

$$
\zeta=\sum_{a: \mathrm{U}(1)} \zeta_{a} h_{a}
$$

i.e. a sum over the Cartan generators $h_{a}$ of the Abelian factors in the gauge group, in terms of the real parameters $\zeta_{a}$. We find the following classes of solutions.

Deformed Coulomb branch. It is characterized by $\phi=0$, therefore from (2.23):

$$
F=\zeta \sin \theta \cos \theta f(\theta) d \theta \wedge(\ell d \varphi-\tilde{\ell} d \chi)
$$

Since $S_{b}^{3}$ has trivial second cohomology, any line bundle is trivial and we can find a globally defined and smooth potential:

$$
A=\zeta[(G(\theta)-G(\pi / 2)) \ell d \varphi+(G(0)-G(\theta)) \tilde{\ell} d \chi]
$$

where $G^{\prime}(\theta)=\sin \theta \cos \theta f(\theta)$. We find

$$
G(\theta)=\frac{\left(\ell^{2}+\tilde{\ell}^{2}-\left(\ell^{2}-\tilde{\ell}^{2}\right) \cos 2 \theta\right)^{3 / 2}}{6 \sqrt{2}\left(\ell^{2}-\tilde{\ell}^{2}\right)}+\text { const, } \quad G\left(\frac{\pi}{2}\right)-G(0)=\frac{\ell^{2}+\ell \tilde{\ell}+\tilde{\ell}^{2}}{3(\ell+\tilde{\ell})}=\frac{\operatorname{vol}\left(S_{b}^{3}\right)}{4 \pi^{2} \ell \tilde{\ell}} .
$$

The scalar $\sigma$ is constant and it commutes with $F$, in particular we can choose a gauge where it is along the Cartan subalgebra.

Higgs-like solutions. They are characterized by $H(\phi)=0$ (we will relax this condition momentarily). This implies $F_{\mu \nu}=0$ and, choosing $A_{\mu}=0$, also $0=\partial_{\mu} \sigma=\partial_{\mu} \phi$ (one has to exclude non-constant solutions for $\phi$ with the same argument as above). Therefore $\sigma$ can be diagonalized, and one is left with the algebraic equations

$$
H(\phi)=0, \quad\left(\sigma+m_{i}\right) \phi_{i}=0 \quad \forall i .
$$


The last equation can be more compactly written as $\mathfrak{S} \phi=0$. These are the standard D-term equations, and their solutions strongly depend on the gauge group and matter content of the theory.

We will be interested in gauge groups and matter representations for which generic parameters $\zeta_{a}$ and generic masses $m_{i}$ lead to solutions to (2.40) that completely break the gauge group. More specifically, we will be focusing on theories for which the Coulomb branch parameters $\sigma_{\alpha}$, for $\alpha=1, \ldots, \operatorname{rank} G$, are fixed (depending on the Higgs-like solution) in terms of the masses $m_{i}$, and for generic masses they are different breaking the gauge group to $\mathrm{U}(1)^{\operatorname{rank} G}$. Each $\mathrm{U}(1)$ is then Higgsed by one component of $\phi$, along a weight $w \in \mathfrak{R}$, getting VEV. One gets a discrete set of Higgs vacua. If the gauge group is not completely broken (including the case of an unbroken discrete gauge group), or if some continuous Higgs branch is left, the situation is more involved and we will not study it here.

Vortices. Each Higgs-like solution is accompanied by a tower of other solutions with arbitrary numbers of vortices at the north and at the south circles (the Higgs-like solution should be thought of as the one with zero vortex numbers). To see this, expand the BPS equations around $\theta=0$ at first order in $\theta$. Defining the coordinate $r=\tilde{\ell} \theta$, the metric reads

$$
d s^{2} \simeq d r^{2}+r^{2} d \chi^{2}+\ell^{2} d \varphi^{2} \quad \text { around } \theta=0
$$

which is $\mathbb{R}^{2} \times S^{1}$. The BPS equations (2.29) and (2.30) reduce to

$$
\begin{aligned}
& r^{-1} F_{r \chi}=-H(\phi) \\
& 0=\left(D_{r}-\frac{i}{r} D_{\chi}\right) \phi \\
& F_{r \varphi}=-\frac{\ell}{\tilde{\ell}} F_{r \chi} \quad F_{\varphi \chi}=0 \\
& D_{\varphi} \phi=-\frac{\ell}{\tilde{\ell}} D_{\chi} \phi \text {. }
\end{aligned}
$$

The two equations on the left are the usual vortex equations ${ }^{8}$ on $\mathbb{R}^{2}$, while the other equations complete the solutions to vortices on $\mathbb{R}^{2} \times S^{1}$ once the solutions on $\mathbb{R}^{2}$ are found. The equations cannot be solved analytically, therefore let us qualitatively describe the solutions in the $\mathrm{U}(1)$ case with a single chiral of charge 1, since - up to a rescaling of the charge - this is the generic situation once the gauge group has been broken to $\mathrm{U}(1)^{\operatorname{rank} G}$ by the VEV of $\sigma$. We take $\zeta>0$, in order to have solutions. Far from the core of the vortex, for $r \gg \sqrt{m / \zeta}$ (the integer $m$ will be defined momentarily), we have $0=H(\phi)=F_{r \chi}=F_{r \varphi}$ therefore

$$
\phi \simeq \sqrt{\zeta} e^{-i n \varphi-i m \chi}, \quad A \simeq-n d \varphi-m d \chi .
$$

Stokes' theorem on $\mathbb{R}^{2}$ implies $\frac{1}{2 \pi} \int F=-m$, i.e. $m$ is the vortex number at the north circle (while $n$ will be interpreted below). At the core of the vortex $\phi$ has to vanish in order to be smooth (if $m \neq 0$ ), therefore close to the core

$$
\begin{aligned}
& \phi \simeq B\left(r e^{-i \chi}\right)^{m} e^{-i n \varphi}, \quad F \simeq \zeta r d r \wedge\left(\frac{\ell}{\tilde{\ell}} d \varphi-d \chi\right) \\
& A \simeq\left(-n-\frac{\ell}{\tilde{\ell}} m+\zeta \frac{\ell}{\tilde{\ell}} \frac{r^{2}}{2}\right) d \varphi-\zeta \frac{r^{2}}{2} d \chi \\
& \text { for } r \ll \sqrt{m / \zeta}
\end{aligned}
$$

\footnotetext{
${ }^{8}$ They are more conventionally antivortex equations, the difference being only the orientation.
} 
where $B$ is some constant. In particular, smoothness of $\phi$ requires $m \in \mathbb{Z}_{\geq 0}$. Note that $\phi$ vanishes only at $r=0$, therefore

$$
\tilde{\ell} A_{\varphi}+\ell A_{\chi}=-\tilde{\ell} n-\ell m
$$

holds exactly. If we approximate $r^{-1} F_{r \chi}$ by a step function on a disk times $-\zeta$, we get that the size of the vortex is of order $\sqrt{m / \zeta}$ justifying the limits we took. In the limit $\zeta \rightarrow \infty$ the vortices squeeze to zero-size, therefore the first-order approximation of the equations around $\theta=0$ is consistent.

We can similarly study the BPS equations expanded around $\theta=\frac{\pi}{2}$ at first order in $\frac{\pi}{2}-\theta$, defining a coordinate $\tilde{r}=\ell\left(\frac{\pi}{2}-\theta\right)$. As before, the equations reduce to the $2 \mathrm{~d}$ antivortex equations (as the orientation induced from $S_{b}^{3}$ is opposite) besides some other equations that complete the solutions to 3 d. For a U(1) gauge theory with a single chiral, the analysis above goes through mutatis mutandis. Far from the core of the vortex, for $\tilde{r} \gg \sqrt{n / \zeta}$, we have the same asymptotic behavior as in (2.43). Stokes' theorem on $\mathbb{R}^{2}$ implies $\frac{1}{2 \pi} \int F=-n$, i.e. $n$ is the antivortex number at the south circle, and the analysis of the solution for $\tilde{r} \ll \sqrt{n / \zeta}$ reveals that $n \in \mathbb{Z}_{\geq 0}$. The behavior of the fields (2.43) in the intermediate region, far from both cores, provides a link of parameters between the two cores and it is indeed a solution of the full BPS equations.

For finite values of $\zeta$, both curvature and finite size effects play a rôle. From the second and third equations on the left in (2.29), integrating over the sphere one can obtain

$$
-4 \pi^{2} \ell \int F_{\theta \chi} d \theta=4 \pi^{2} \tilde{\ell} \int F_{\theta \varphi} d \theta=\int H(\phi) d \operatorname{vol}_{S_{b}^{3}} \leq \zeta \operatorname{vol}\left(S_{b}^{3}\right),
$$

where we used that $H(\phi)$ is bounded by $0 \leq H(\phi) \leq \zeta$ on vortex solutions, and vortex solutions have only $\theta$ dependence. Still working in a gauge with smooth and globally defined connection $A$, we can define the vortex numbers $m, n$ at the north and south circle as the winding numbers of $\phi$ around $\chi, \varphi$ respectively. The analyses at the cores are still valid, therefore $m, n \in \mathbb{Z}_{\geq} 0$ and

$$
-\frac{A_{\varphi}(0)}{\ell}=-\frac{A_{\chi}\left(\frac{\pi}{2}\right)}{\tilde{\ell}}=\frac{n}{\ell}+\frac{m}{\tilde{\ell}} .
$$

Then the bound above implies a bound on the vortex and antivortex numbers:

$$
b n+b^{-1} m \leq \zeta \frac{\operatorname{vol}\left(S_{b}^{3}\right)}{4 \pi^{2} \sqrt{\ell \tilde{\ell}}} .
$$

We conclude that for finite values of $\zeta$ there is a finite number of vortex/antivortex solutions on the squashed three-sphere; when the bound is saturated, the chiral field $\phi$ actually vanishes and the gauge field is as in the deformed Coulomb branch described before. We thus get a nice picture of the structure of solutions as we continuously increase $\zeta$ from 0 to $+\infty$. The Coulomb branch solution is continuously deformed into the deformed Coulomb branch solution; as $\zeta$ crosses one of the thresholds, proportional to $b n+b^{-1} m$, a new (anti)vortex solution branches out, in which the value of the matter field is infinitesimal at 
the threshold and increases further on. This picture will be useful in the next section to understand how localization changes as we change $\zeta$ continuously.

For gauge groups of rank larger than one, there can be mixed Coulomb-Higgs branches where part of the gauge group is broken to a diagonal torus (along those components BPS solutions describe a deformed Coulomb branch) and part is completely broken (admitting vortex solutions).

\subsection{Computation of the partition function}

Given the various classes of solutions to the BPS equations found in the previous section, the computation of the partition function requires two more steps: the evaluation of the classical action and of the one-loop determinant of quadratic fluctuations around the BPS configurations, and the sum/integration over the space of BPS configurations.

\subsubsection{One-loop determinants from an index theorem}

For the computation of the one-loop determinants around non-constant configurations, one most conveniently makes use of an equivariant index theorem for transversally elliptic operators [45], as in [46]. A similar technique has been used on $S^{4}[3,47]$ and $S^{2}$ [4]. One can give a cohomological form to the $\mathcal{Q}$-exact localizing action (this point is well explained in $[3,47])$, and, with the equivariant index theorem, the one-loop determinants of quadratic fluctuations only get contributions from the fixed points of the equivariant rotations on the worldvolume. Recall that the localizing supercharge squares to

$$
\mathcal{Q}^{2}=\mathcal{L}_{\xi}^{A}-\mathfrak{S}-\frac{i}{2}\left(\frac{1}{\ell}+\frac{1}{\tilde{\ell}}\right) R
$$

The vector field $\xi=\frac{1}{\ell} \partial_{\varphi}+\frac{1}{\tilde{\ell}} \partial_{\chi}$ does not have fixed points on $S_{b}^{3}$, on the other hand its orbits do not close for generic values of $b$ ( $\xi$ generates a non-compact isometry group $\mathbb{R}$ ) and since the index theorem requires a compact group action, we cannot use it directly. ${ }^{9}$ The idea of [46] is to write $\xi=\left(\frac{1}{\ell}+\frac{1}{\ell}\right) \partial_{\psi_{H}}+\left(\frac{1}{\ell}-\frac{1}{\ell}\right) \partial_{\phi_{H}}$ in Hopf coordinates: it generates a free rotation of the Hopf fiber and a rotation of the base space. We can reduce the operator for quadratic fluctuations (i.e. the operator resulting from the quadratic expansion of the localizing action around the background) along the Hopf fiber, obtaining a transversally elliptic operator on the base $S^{2}$. We thus reduce the problem to the computation of a one-loop determinant on the base $S^{2}$, dressed by the KK modes on the Hopf fiber. The projection of $\xi$ to $S^{2}$ gives a rotation with fixed points at $\theta=0$ (which we call North) and $\theta=\frac{\pi}{2}$ (which we call South). This is exactly the setup in [4]. Identifying the equivariant parameters of the $\mathrm{U}(1)_{\partial_{\phi_{H}}} \times \mathrm{U}(1)_{R} \times G$ action as $\varepsilon=\frac{1}{\ell}-\frac{1}{\tilde{\ell}}, \check{\varepsilon}=\frac{1}{\ell}+\frac{1}{\tilde{\ell}}$ and $a=-i\left(\frac{1}{\ell} A_{\varphi}+\frac{1}{\tilde{\ell}} A_{\chi}\right)-\mathfrak{S}$, following [46] we obtain (see appendix $\mathrm{C}$ ) the one-loop determinant for a chiral multiplet of R-charge $q$ in gauge representation $\mathcal{R}$ :

$$
Z_{1-\text { loop }}^{\text {chiral }}=" \prod_{w \in \mathcal{R}} \prod_{n \in \mathbb{Z}} \prod_{m \geq 0} \frac{(m+1) \ell^{-1}+n \tilde{\ell}^{-1}-\frac{q}{2} \check{\varepsilon}-i w\left(a_{S}\right)}{n \ell^{-1}-m \tilde{\ell}^{-1}-\frac{q}{2} \check{\varepsilon}-i w\left(a_{N}\right)} .
$$

\footnotetext{
${ }^{9}$ For special values of $b$, e.g. the round sphere $b=1$, the group action is a compact $\mathrm{U}(1)$. Still the index theorem determines the index up to torsion, and in fact in those cases the index turns out to be pure torsion. We thank Takuya Okuda for correspondence on this issue.
} 
In all BPS configurations that we consider in this section, $a_{N}=a_{S} \equiv a$ and some further simplifications take place. It is also convenient to introduce the rescaled variable $\hat{a} \equiv \sqrt{\ell \tilde{\ell}} a$, as well as $b \equiv \sqrt{\tilde{\ell} / \ell}$ and $Q=b+b^{-1}$. Rescaling numerator and denominator of (2.50) by $\sqrt{\ell \tilde{\ell}}$ and neglecting overall signs, we are led to

$$
Z_{1 \text {-loop }}^{\text {chiral }}=" \prod_{w \in \mathcal{R}} \prod_{m, n \geq 0} \frac{m b+n b^{-1}+\left(1-\frac{q}{2}\right) Q-i w(\hat{a})}{m b+n b^{-1}+\frac{q}{2} Q+i w(\hat{a})}=\prod_{w \in \mathcal{R}} s_{b}\left(\frac{i Q}{2}(1-q)+w(\hat{a})\right) .
$$

The last one is the regulated expression found in [12], in terms of the double sine function $s_{b}$. The one-loop determinant for the vector multiplet is simply

$$
Z_{1 \text { loop }}^{\text {vec }}=\prod_{\alpha>0} 2 \sinh (\pi b \alpha(\hat{a})) 2 \sinh \left(\pi b^{-1} \alpha(\hat{a})\right),
$$

where the product is over the positive roots $\alpha$ of the gauge group.

\subsubsection{Coulomb branch}

Let us first quickly review the Coulomb branch localization formula, obtained by choosing $\zeta=0$ in $H(\phi)$, or taking positive R-charges. The matrix model was derived in [12]. The only $\mathcal{Q}$-closed but not $\mathcal{Q}$-exact pieces of classical action are the CS and FI terms (that we report in appendix B.3). Evaluation on the Coulomb branch configurations gives

$$
S_{\mathrm{cl}}=i \pi \operatorname{Tr}_{\mathrm{CS}} \hat{\sigma}^{2}-2 \pi i \operatorname{Tr}_{\mathrm{FI}} \hat{\sigma}
$$

in terms of the rescaled adjoint scalar $\hat{\sigma} \equiv \sqrt{\ell \tilde{\ell}} \sigma$. The weighted traces $\operatorname{Tr}_{\mathrm{CS}}$ and $\operatorname{Tr}_{\mathrm{FI}}$ are spelled out in appendix B.3, and for $\mathrm{U}(N)$ at level $k$ they reduce to $S_{\mathrm{cl}}=i \pi k \operatorname{Tr} \hat{\sigma}^{2}-$ $2 \pi i \xi \operatorname{Tr} \hat{\sigma}$.

Since the equivariant parameters for gauge transformations are equal at the two fixed circles, $\hat{a}_{N}=\hat{a}_{S}=-\hat{\sigma}$, the one-loop determinants (2.51) and (2.52) are

$$
Z_{1-\text { loop }}^{\text {chiral }}=\prod_{w \in \mathcal{R}} s_{b}\left(\frac{i Q}{2}(1-q)-w(\hat{\sigma})\right), \quad Z_{1 \text {-loop }}^{\mathrm{vec}}=\prod_{\alpha>0} 2 \sinh (\pi b \alpha(\hat{\sigma})) 2 \sinh \left(\pi b^{-1} \alpha(\hat{\sigma})\right) .
$$

This leads to the matrix integral of [12]:

$$
Z_{S_{b}^{3}}=\frac{1}{|\mathcal{W}|} \int\left(\prod_{a=1}^{\mathrm{rank} G} d \hat{\sigma}_{a}\right) e^{-i \pi \operatorname{Tr}_{\mathrm{CS}} \hat{\sigma}^{2}+2 \pi i \operatorname{Tr}_{\mathrm{FI}} \hat{\sigma}} Z_{1-\text { loop }}^{\text {vec }} Z_{1 \text {-loop }}^{\text {chiral }}
$$

where $|\mathcal{W}|$ is the dimension of the Weyl group. Notice that the Vandermonde determinant for integration over the gauge algebra $\mathfrak{g}$ cancels against the one-loop determinant for gaugefixing ghosts.

\subsubsection{Deformed Coulomb branch}

Let us now study the contributions for $\zeta \neq 0$. The classical CS and FI actions evaluated on the deformed Coulomb branch configurations give

$$
S_{\mathrm{cl}}^{\mathrm{CS}}=i \pi \operatorname{Tr}_{\mathrm{CS}}(\hat{\sigma}-i \zeta \kappa)^{2}, \quad S_{\mathrm{cl}}^{\mathrm{FI}}=-2 \pi i \operatorname{Tr}_{\mathrm{FI}}(\hat{\sigma}-i \zeta \kappa)
$$


and we defined the constant

$$
\kappa \equiv \frac{\operatorname{vol}\left(S_{b}^{3}\right)}{4 \pi^{2} r}=\frac{r^{2}}{3}\left(Q-Q^{-1}\right)=\frac{r}{3} \frac{\ell^{2}+\tilde{\ell}+\tilde{\ell}^{2}}{\ell+\tilde{\ell}},
$$

where $r \equiv \sqrt{\ell \tilde{\ell}}$. In both cases the effect of the deformation parameter $\zeta$ is effectively to shift the integration variable $\hat{\sigma}$ in the imaginary direction. The same shift occurs in the equivariant gauge parameters

$$
\hat{a}_{N}=\hat{a}_{S}=-\hat{\mathfrak{S}}+i \zeta \kappa
$$

defined above (2.50), as it follows from (2.38), and so also the one-loop determinants simply suffer an effective imaginary shift of $\hat{\sigma}$. Therefore the whole deformed Coulomb branch contribution is simply obtained from the undeformed Coulomb branch expression (2.55) by shifting the integration contours in the imaginary directions.

Since the parameter $\zeta$ was introduced via a $\mathcal{Q}$-exact term in the action, the partition function should not depend on it. For $\zeta=0$ we have the original Coulomb branch integral (2.55). Upon turning on $\zeta$ we effectively deform the contours, shifting them in the imaginary directions, and the integral remains constant until we cross some pole of the chiral one-loop determinant. One can anticipate what happens when crossing a pole based on the bound (2.48): the imaginary coordinates of the poles precisely correspond to values of $\zeta$ for which new vortices appear on $S_{b}^{3}$ as solutions to the vortex equations, and the contribution from the vortices precisely accounts for the jumps in the deformed Coulomb branch integral.

Suppression. Our goal is to derive a localization procedure that reduces the partition function to a pure sum over vortices, with no spurious contributions from deformed Coulomb branches. In order to do that, we can take a suitable limit $\zeta_{a} \rightarrow \pm \infty$ : in favorable situations, there exists (for a choice of signs) a limit in which the deformed Coulomb branch contribution vanishes.

Let us define the $\mathrm{U}(1)$ charges of a gauge representation $\mathcal{R}_{j}: \mathfrak{q}_{j}^{(a)} \equiv w\left(h_{a}\right)$, where $h_{a}$ are the Cartan generators of the Abelian factors in the gauge group, as in (2.36), while $w$ is any one weight of $\mathcal{R}_{j} .{ }^{10}$ We also decompose $\hat{\sigma}=\hat{\sigma}_{R}-i \zeta \kappa$ into its real and imaginary parts. Using the asymptotic behavior of the double-sine function (see e.g. the appendix of $[48])$ :

$$
s_{b}(z) \rightarrow \begin{cases}e^{+i \frac{\pi}{2}\left(z^{2}+\frac{1}{12}\left(b^{2}+b^{-2}\right)\right)} & |z| \rightarrow \infty, \quad|\arg z|<\frac{\pi}{2} \\ e^{-i \frac{\pi}{2}\left(z^{2}+\frac{1}{12}\left(b^{2}+b^{-2}\right)\right)} & |z| \rightarrow \infty, \quad|\arg z|>\frac{\pi}{2},\end{cases}
$$

one finds that the absolute value of the integrand in the partition function matrix model has the following suppression factor, for $\zeta_{a} \rightarrow \pm \infty$ :

$$
\mid \text { integrand } \mid \sim \exp \left[-2 \pi \kappa \sum_{a} \zeta_{a}\left(\operatorname{Tr}_{\mathrm{CS}} \hat{\sigma}_{R} h_{a}-\operatorname{Tr}_{\mathrm{FI}} h_{a}+\frac{1}{2} \sum_{\mathcal{R}_{j}} \mathfrak{q}_{j}^{(a)} \sum_{w \in \mathcal{R}_{j}}\left|w\left(\hat{\sigma}_{R}\right)+m_{j}\right|\right)\right],
$$

\footnotetext{
${ }^{10}$ There is no dependence on the particular weight $w$ chosen, since the $\mathrm{U}(1)$ generators commute with all roots of the simple factors.
} 
where the first two terms in parenthesis originate from the classical action while the last term comes from the chiral multiplets in those representations $\mathcal{R}_{j}$ with $\mathfrak{q}_{j}^{(a)} \neq 0$. The one-loop determinants of chiral multiplets with $\mathfrak{q}_{j}^{(a)}=0$ and that of vector multiplets are unaffected by $\zeta$. One can achieve a suppression of the deformed Coulomb branch contribution if there exists a choice of signs in the limit $\zeta_{a} \rightarrow \pm \infty$ such that the factor above goes to zero for all values of all components of $\hat{\sigma}_{R}$.

As a concrete example, consider a $\mathrm{U}(N)$ theory with $N_{f}$ fundamentals, $N_{a}$ antifundamentals and some adjoint chiral multiplets (there is a single Abelian factor in the gauge group, and $\mathfrak{q}$ equals $1,-1$ and 0 respectively). Setting the real masses to zero for simplicity, the factor above provides a suppression of the deformed Coulomb branch for

$$
\zeta \rightarrow+\infty \quad \text { and } \quad-\frac{N_{f}-N_{a}}{2}<k<\frac{N_{f}-N_{a}}{2},
$$

in particular $N_{f}>N_{a}$, where the two constraints come from positive and negative $\hat{\sigma}_{R}$. Similarly, we have suppression for

$$
\zeta \rightarrow-\infty \quad \text { and } \quad \frac{N_{f}-N_{a}}{2}<k<-\frac{N_{f}-N_{a}}{2},
$$

In particular $N_{a}>N_{f}$. These two cases, $|k|<\left|N_{f}-N_{a}\right| / 2$, are the "maximally chiral" theories of [49]. In case one or both bounds are saturated, then the true FI term $\xi$ needs to have the correct sign.

We stress that if the "maximally chiral" condition (including saturations of the bounds) is not met, i.e. if $|k| \leq\left|N_{f}-N_{a}\right| / 2$ is not met, the deformed Coulomb branch contribution is not suppressed. As we will see in the next section, this translates to the fact that the Coulomb branch integral cannot be closed neither in the upper nor lower half-plane, and reduction to a sum over residues (as in [23]) requires some more clever procedure (if possible at all).

\subsubsection{Higgs branch and vortex partition function}

For finite values of the deformation parameters $\zeta_{a}$, among the BPS configurations of section 2.3 we find Higgs vacua and vortex solutions, where the (anti)vortex numbers $(m, n)$ are bounded by (2.48) (or its multi-dimensional version). These BPS configurations contribute to the path integral, besides the deformed Coulomb branch discussed before. Let us determine their contribution.

The classical actions can be integrated exactly (even though the vortex solutions cannot be written explicitly) using $D=-\sigma / f+i H(\phi)$, the BPS equations (2.29) and the knowledge of $A_{\varphi}(\theta)$ at $\theta=0, \frac{\pi}{2}$ in a globally defined gauge with $A_{\theta}=0$, as discussed around (2.44). One finds

$$
S_{\mathrm{cl}}^{\mathrm{CS}}=i \pi \operatorname{Tr}_{\mathrm{CS}}\left(\hat{\sigma}-i b^{-1} m-i b n\right)^{2}, \quad S_{\mathrm{cl}}^{\mathrm{FI}}=-2 \pi i \operatorname{Tr}_{\mathrm{FI}}\left(\hat{\sigma}-i b^{-1} m-i b n\right) .
$$

Here the vortex numbers $m, n$ should really be thought of as GNO quantized [50] elements of the gauge algebra, i.e. belonging to the coweight lattice. 
The evaluation of the one-loop determinants for the off-diagonal W-bosons and all chiral multiplets not getting a VEV is straightforward: one identifies the equivariant gauge transformation parameters in the vortex background from the expression of $\mathcal{Q}^{2}$ at the poles:

$$
\hat{a}_{N}=\hat{a}_{S}=-\left(\hat{\mathfrak{S}}-i b^{-1} m-i b n\right) .
$$

These values have to be plugged into (2.51) and (2.52). For the rank $G$ chiral multiplets that get a VEV and, by Higgs mechanism, pair with the vector multiplets along the maximal torus of the gauge group becoming massive, one has to be more careful. As pointed out in [5], the one-loop determinant for the combined system is just the residue of the chiral one-loop. ${ }^{11}$ Therefore the total contribution from the chiral multiplets is

$$
Z_{1-\text { loop }}^{\text {chiral }}=\operatorname{Res}_{\mathfrak{S} \rightarrow \mathfrak{S}_{H}} \prod_{w \in \Re} s_{b}\left(\frac{i Q}{2}-w\left(\hat{\mathfrak{S}}-i m b^{-1}-i n b\right)\right),
$$

where $\mathfrak{S}_{H}$ denotes $\mathfrak{S}$ evaluated on the particular Higgs vacuum, and the R-charges have been set to zero. Finally, since each BPS solution is a smooth configuration with no moduli, we simply sum over them with weight 1 .

From (2.63) it is clear that the sum of the contributions from the finite number of vortices satisfying the bound (2.48) exactly accounts for the jumps in the deformed Coulomb branch contribution every time the integration contour - which is shifted in the imaginary directions by $\zeta_{a}$-crosses a pole of the chiral one-loop determinant. This of course is expected, since the path integral should not depend on $\zeta$.

Vortex partition function. We obtain a more interesting result if we take a suitable $\zeta_{a} \rightarrow \pm \infty$ limit in which the deformed Coulomb branch contribution vanishes, and there is no bound on the (anti)vortex numbers. Conditions for the existence of such a limit were discussed in section 2.4.3.

In this limit the path integral is completely dominated by (anti)vortex-string configurations wrapping the northern and southern circles, and whose size shrinks to zero. The resummed contribution of all vortex strings is accounted by the K-theoretic vortex partition function, $Z_{\text {vortex }}$, which can be computed on the twisted $\mathbb{R}_{\epsilon}^{2} \times S^{1}: \mathbb{R}^{2}$ is rotated by the equivariant parameter $\epsilon$ as we go around $S^{1}$, and this effectively compactifies the space. In fact one associates equivariant parameters to flavor symmetries as well. In a suitable scaling limit in which $S^{1}$ shrinks (together with the equivariant parameters), one recovers the vortex partition function in $\Omega$-background of [25]. This all is the $2 \mathrm{~d}$ analog of the $4 \mathrm{~d}$ and $5 \mathrm{~d}$ instanton partition functions constructed in $[26,36]$.

Let us compute the partition function in this limit. First, we have a finite number of Higgs vacua. In each vacuum, $\hat{\sigma}_{\alpha}$ are fixed to some specific (real) values that are functions of the real masses. The classical actions (2.61) provide a weighting factor to $Z_{\text {vortex }}$ for the vortex configurations, times an overall classical contribution:

$$
S_{\mathrm{cl}}=i \pi \operatorname{Tr}_{\mathrm{CS}} \hat{\sigma}^{2}-2 \pi i \operatorname{Tr}_{\mathrm{FI}} \hat{\sigma} .
$$

\footnotetext{
${ }^{11}$ The chiral one-loop diverges because it is evaluated at a point on the Coulomb branch where the chiral multiplet, before pairing with the vector multiplet, is massless. Taking the residue corresponds to removing the zero-mode.
} 
The weighting factors for (anti)vortices have a term quadratic in the vortex number and a linear term:

$$
\begin{aligned}
e^{-S_{\mathrm{v}}} & =\exp \left[i \pi b^{-2} \operatorname{Tr}_{\mathrm{CS}} m^{2}+2 \pi b^{-1}\left(-\operatorname{Tr}_{\mathrm{CS}} \hat{\sigma} \cdot+\operatorname{Tr}_{\mathrm{FI}}\right) m\right] \\
e^{-S_{\mathrm{av}}} & =\exp \left[i \pi b^{2} \operatorname{Tr}_{\mathrm{CS}} n^{2}+2 \pi b\left(-\operatorname{Tr}_{\mathrm{CS}} \hat{\sigma} \cdot+\operatorname{Tr}_{\mathrm{FI}}\right) n\right] .
\end{aligned}
$$

The actions (2.61) also give rise to a term $e^{2 \pi i \operatorname{Tr}_{C S} m n}$ : in the absence of parity anomaly in the matter sector, $\operatorname{Tr}_{C S} m n$ is integer and the term equals 1; otherwise $\operatorname{Tr}_{C S}$ is semi-integer and such that the term is a sign precisely canceling the parity anomaly. ${ }^{12}$

Second, the one-loop determinants for the vector multiplet and the chiral multiplets not acquiring a VEV are as in (2.54). The $\operatorname{rank} G$ chiral multiplets acquiring VEV bring a residue factor, which in this case is just 1 . Finally, the vortex partition function $Z_{\text {vortex }}$ depends on equivariant parameters for rotations of $\mathbb{R}^{2}(\varepsilon)$ and flavor rotations $(g)$ : they are identified - at $\theta=0(\mathrm{~N})$ and $\theta=\frac{\pi}{2}(\mathrm{~S})$ - from the $\mathrm{SU}(1 \mid 1)$ complex of the supercharge $\mathcal{Q}$ at the poles, i.e. from $\mathcal{Q}^{2}$ in (2.49). We find

$$
\varepsilon_{N}=\frac{2 \pi}{b^{2}}, \quad g_{N}=-\frac{2 \pi}{b} \hat{\mathfrak{S}}, \quad \quad \varepsilon_{S}=2 \pi b^{2}, \quad g_{S}=-2 \pi b \hat{\mathfrak{S}}
$$

Eventually, Higgs branch localization gives the following expression of the sphere partition function:

$$
Z_{S_{b}^{3}}=\sum_{\text {Higgs vacua }} e^{-i \pi \operatorname{Tr}_{\mathrm{CS}} \hat{\sigma}^{2}+2 \pi i \operatorname{Tr}_{\mathrm{FI}} \hat{\sigma}} Z_{1 \text {-loop }}^{\prime} Z_{\mathrm{v}} Z_{\mathrm{av}}
$$

The sum is over solutions to (2.40). The one-loop determinant $Z_{1 \text {-loop }}^{\prime}$ does not contain the rank $G$ chiral multiplets getting VEV in (2.40). The (anti)vortex-string contributions are expressed in terms of the $3 \mathrm{~d}$ vortex partition function:

$$
\begin{aligned}
& Z_{\mathrm{v}}=Z_{\mathrm{vortex}}\left(e^{i \pi b^{-2} \operatorname{Tr}_{\mathrm{CS}} \cdot}, e^{2 \pi b^{-1}\left(-\operatorname{Tr}_{\mathrm{CS}} \hat{\sigma} \cdot+\operatorname{Tr}_{\mathrm{FI}} \cdot\right)}, \frac{2 \pi}{b^{2}},-\frac{2 \pi}{b} \hat{\mathfrak{S}}\right) \\
& Z_{\mathrm{av}}=Z_{\mathrm{vortex}}\left(e^{i \pi b^{2} \operatorname{Tr}_{\mathrm{CS}} \cdot}, e^{2 \pi b\left(-\operatorname{Tr}_{\mathrm{CS}} \hat{\sigma} \cdot+\operatorname{Tr}_{\mathrm{FI}} \cdot\right)}, 2 \pi b^{2},-2 \pi b \hat{\mathfrak{S}}\right) .
\end{aligned}
$$

The first two arguments in the vortex partition function are exponentiated linear functions on the gauge algebra, corresponding to the quadratic and linear weights for the vortex numbers; the third is the rotational equivariant parameter and the last one includes all flavor equivariant parameters. Notice that the expression (2.67) is very much in the spirit of the "holomorphic blocks" of [28].

We shall give a concrete example in the next section.

\subsection{Matching with the Coulomb branch integral}

We would like to briefly show, in the simple example of a $\mathrm{U}(N)$ gauge theory with $N_{f}$ fundamentals and $N_{a}$ antifundamentals, that Higgs branch and Coulomb branch localization produce in fact the same partition function, written in a completely different way. This

\footnotetext{
${ }^{12}$ Concretely, for $\mathrm{U}(N)_{k}$ with $N_{f}$ fundamentals and $N_{a}$ antifundamentals, cancelation of the parity anomaly requires $2 k+N_{f}-N_{a} \in \mathbb{Z}$. The general case is discussed in [51].
} 
computation has already been done in the case of $\mathrm{U}(1)$ in [23], and in the case of $\mathrm{U}(N)$ in [29], therefore we will just review it in our conventions.

The theory has $\mathrm{SU}\left(N_{f}\right) \times \mathrm{SU}\left(N_{a}\right) \times \mathrm{U}(1)_{A}$ flavor symmetry. We will use a "quiver" notation, in which the fundamentals are in the antifundamental representation of the flavor group $\mathrm{SU}\left(N_{f}\right)$, and viceversa. Then we can introduce real masses $m_{\alpha}$ for fundamentals and $\tilde{m}_{\beta}$ for antifundamentals, defined up to a common shift (which corresponds to a shift of the adjoint scalar $\sigma$ ). Generic positive R-charges are encoded as imaginary parts of the masses.

The matrix integral $(2.55)$ is given by (we removed hat from $\hat{\sigma}$ ):

$$
\begin{aligned}
Z_{S_{b}^{3}}^{\mathrm{U}(N), N_{f}, N_{a}}= & \frac{1}{N !} \int d^{N} \sigma e^{-i \pi k \sum \sigma_{i}^{2}+2 \pi i \xi \sum \sigma_{i}} \prod_{i<j}^{N} 4 \sinh \left(\pi b^{-1}\left(\sigma_{i}-\sigma_{j}\right)\right) \sinh \left(\pi b\left(\sigma_{i}-\sigma_{j}\right)\right) \\
& \times \prod_{i=1}^{N} \frac{\prod_{\beta=1}^{N_{a}} s_{b}\left(\frac{i Q}{2}+\sigma_{i}-\tilde{m}_{\beta}\right)}{\prod_{\alpha=1}^{N_{f}} s_{b}\left(-\frac{i Q}{2}+\sigma_{i}-m_{\alpha}\right)},
\end{aligned}
$$

where we used $s_{b}(-x)=s_{b}^{-1}(x)$. Our goal is to rewrite it as a sum over residues, as done in $[23,29]$. First, one can employ twice the Cauchy determinant formula that we use in the following form:

$$
\prod_{i<j}^{N} 2 \sinh \left(x_{i}-x_{j}\right)=\frac{1}{\prod_{i<j}^{N} 2 \sinh \left(\chi_{i}-\chi_{j}\right)} \sum_{s \in S^{N}}(-1)^{s} \prod_{i=1}^{N} \prod_{j \neq s(i)}^{N} 2 \cosh \left(x_{i}-\chi_{j}\right),
$$

where the auxiliary variables $\chi_{i}$ must satisfy $\chi_{i} \neq \chi_{j}(\bmod \pi i)$, to separate the interacting matrix-model into a product of simple integrals. The simple integrals will contain two sets of auxiliary variables $\chi_{i}, \tilde{\chi}_{i}$. Assuming that $|k|<\frac{N_{f}-N_{a}}{2}$ (or $|k| \leq \frac{N_{f}-N_{a}}{2}$ and $\xi<0$ ), these integrals can be computed by closing the contour in the lower-half plane and then picking up the residues. The regime $|k| \leq \frac{N_{a}-N_{f}}{2}$ can be studied in a similar way, closing the contours in the upper-half plane. One gets contributions from the simple poles of the one-loop determinants of fundamentals, located at the zeros of $s_{b}$ in the denominator: $\sigma_{j}=$ $m_{\gamma_{j}}-i \mu_{j} b-i \nu_{j} b^{-1} \equiv \tau_{j}\left(m_{\gamma_{j}}, \mu_{j}, \nu_{j}\right)$ for $\mu_{j}, \nu_{j} \in \mathbb{Z}_{\geq 0}$ and $\gamma_{j}=1, \ldots, N_{f}$. Applying the Cauchy determinant formula backwards, to re-absorb the auxiliary variables, one obtains

$$
\begin{aligned}
Z_{S_{b}^{3}}= & \frac{(-2 \pi i)^{N}}{N !} \sum_{\vec{\gamma} \in\left(\mathbb{Z}_{N_{f}}\right)^{N}} \sum_{\vec{\mu}, \vec{\nu} \in \mathbb{Z}_{\geq 0}^{N}} e^{-i \pi k \sum \tau_{i}^{2}+2 \pi i \xi \sum \tau_{i}} \prod_{i<j}^{N} 4 \sinh \left(\pi b\left(\tau_{i}-\tau_{j}\right)\right) \sinh \left(\pi b^{-1}\left(\tau_{i}-\tau_{j}\right)\right) \\
& \times \prod_{i=1}^{N}\left(\frac{\prod_{\beta=1}^{N_{a}} s_{b}\left(\frac{i Q}{2}+\tau_{i}-\tilde{m}_{\beta}\right)}{\prod_{\alpha \neq \gamma_{i}}^{N_{f}} s_{b}\left(-\frac{i Q}{2}+\tau_{i}-m_{\alpha}\right)} \operatorname{Res}_{x \rightarrow 0} s_{b}\left(\frac{i Q}{2}+i \mu_{i} b+i \nu_{i} b^{-1}-x\right)\right)
\end{aligned}
$$

Of course, one could have just collected the residues of the multi-dimensional integral with no need of the Cauchy formula. The residue can be computed with the identity

$$
s_{b}\left(x+\frac{i Q}{2}+i \mu b+i \nu b^{-1}\right)=\frac{(-1)^{\mu \nu} s_{b}\left(x+\frac{i Q}{2}\right)}{\prod_{\lambda=1}^{\mu} 2 i \sinh \pi b(x+i \lambda b) \prod_{\kappa=1}^{\nu} 2 i \sinh \pi b^{-1}\left(x+i \kappa b^{-1}\right)}
$$


and $\operatorname{Res}_{x \rightarrow 0} s_{b}(x+i Q / 2)=1 / 2 \pi i$. At this point one can factorize the summation into a factor independent of $\vec{\mu}$ and $\vec{\nu}$, a summation over $\vec{\mu}$ and a summation over $\vec{\nu}$. To achieve that one uses $2 k+N_{f}-N_{a}=0(\bmod 2)$, which is the condition for parity anomaly cancelation, so that $(-1)^{\left(N_{f}-N_{a}+2 k\right) \sum_{i} \mu_{i} \nu_{i}}=1$. Finally one observes that each of the two summations over $\vec{\mu}$ and $\vec{\nu}$ vanishes if we choose $\gamma_{i}=\gamma_{j}$ for some $i, j$, and on the other hand it is symmetric under permutations of the $\gamma_{i}$ 's. Therefore we can restrict the sum over unordered combinations $\vec{\gamma} \in C\left(N, N_{f}\right)$ of $N$ out of the $N_{f}$ flavors, and cancel the $N$ ! in the denominator.

We can also use the following identity (see e.g. appendix B of [31]), valid when the $\gamma_{i}^{\prime}$ 's are distinct:

$$
\begin{aligned}
& \frac{\prod_{j<k}^{N} \sinh \left(X_{\gamma_{k}}-X_{\gamma_{j}}+i\left(\mu_{k}-\mu_{j}\right) Y\right)}{\prod_{i=1}^{N} \prod_{\beta=1}^{N_{f}} \prod_{\lambda=1}^{\mu_{i}} \sinh \left(X_{\gamma_{i}}-X_{\beta}+i \lambda Y\right)}= \\
& =\frac{(-1)^{\sum_{j} \mu_{j}} \prod_{j<k}^{N} \sinh \left(X_{\gamma_{k}}-X_{\gamma_{j}}\right)}{\prod_{k=1}^{N} \prod_{\lambda=1}^{\mu_{k}}\left[\prod_{j=1}^{N} \sinh \left(X_{\gamma_{k}}-X_{\gamma_{j}}-i\left(\mu_{j}-\lambda+1\right) Y\right)\right]\left[\prod_{\beta \notin\left\{\gamma_{l}\right\}}^{N_{f}} \sinh \left(X_{\gamma_{i}}-X_{\beta}+i \lambda Y\right)\right]}
\end{aligned}
$$

and the observation $\prod_{i<j}^{N}(-1)^{\mu_{i}-\mu_{j}}=(-1)^{(N-1) \sum_{i} \mu_{i}}$, to eventually write:

$$
Z_{S_{b}^{3}}=\sum_{\vec{\gamma} \in C\left(N, N_{f}\right)} Z_{\mathrm{cl}}^{(\vec{\gamma})} Z_{1-\text { loop }}^{\prime(\vec{\gamma})} Z_{\mathrm{v}}^{(\vec{\gamma})} Z_{\mathrm{av}}^{(\vec{\gamma})}
$$

which exactly matches with the general result of Higgs branch localization (2.67). The summation is over classical Higgs vacua, i.e. over solutions to the algebraic D-term equations (2.40). Then we have a simple classical piece, the one-loop determinant of all fields except the $N$ chiral multiplets (specified by $\vec{\gamma}$ ) getting a VEV and Higgsing the gauge group, the vortex and the anti-vortex contributions; all these functions are evaluated at the point $(\vec{\gamma})$ on the Coulomb branch solving the D-term equations. Using a notation in which $\alpha \in \vec{\gamma}$ denotes the flavor indices in the combination $\vec{\gamma}$, we can write the classical and one-loop contributions as

$$
\begin{aligned}
Z_{\mathrm{cl}}^{(\vec{\gamma})} & =\prod_{\alpha \in \vec{\gamma}} e^{-i \pi k m_{\alpha}^{2}+2 \pi i \xi m_{\alpha}} \\
Z_{1-\text { loop }}^{\prime(\vec{\gamma})} & =\prod_{i \in \vec{\gamma}} \frac{\prod_{\beta=1}^{N_{a}} s_{b}\left(\frac{i Q}{2}+m_{i}-\tilde{m}_{\beta}\right)}{\prod_{\alpha(\neq i)}^{N_{f}} s_{b}\left(-\frac{i Q}{2}+m_{i}-m_{\alpha}\right)} \cdot \prod_{\substack{i, j \in \vec{\gamma} \\
i \neq j}} 4 \sinh \left(\pi b\left(m_{i}-m_{j}\right)\right) \sinh \left(\pi b^{-1}\left(m_{i}-m_{j}\right)\right),
\end{aligned}
$$

the (anti)vortex contributions as

$$
\begin{aligned}
& Z_{\mathrm{v}}^{(\vec{\gamma})}=Z_{\mathrm{vortex}}^{(\vec{\gamma})}\left(e^{i \pi b^{-2} k},\left.e^{2 \pi b^{-1}\left(-k m_{j}+\xi\right)}\right|_{j \in \vec{\gamma}}, \frac{2 \pi}{b^{2}},-\frac{2 \pi}{b} m_{\alpha},-\frac{2 \pi}{b} \tilde{m}_{\beta}\right) \\
& Z_{\mathrm{av}}^{(\vec{\gamma})}=Z_{\mathrm{vortex}}^{(\vec{\gamma})}\left(e^{i \pi b^{2} k},\left.e^{2 \pi b\left(-k m_{j}+\xi\right)}\right|_{j \in \vec{\gamma}}, 2 \pi b^{2},-2 \pi b m_{\alpha},-2 \pi b \tilde{m}_{\beta}\right),
\end{aligned}
$$


and the vortex-string partition function turns out to be (for $N_{f} \geq N_{a}$ ):

$$
\begin{aligned}
& Z_{\text {vortex }}^{(\vec{\gamma})}\left(Q_{j}, L_{j}, \varepsilon, a_{\alpha}, b_{\beta}\right)=\sum_{\vec{\mu} \in \mathbb{Z}_{\geq 0}^{N}} \prod_{j \in \vec{\gamma}} Q_{j}^{\mu_{j}^{2}} L_{j}^{\mu_{j}}(-1)^{\left(N_{f}-N_{a}\right) \mu_{j}} \\
& \quad \times \prod_{\lambda=0}^{\mu_{j}-1} \frac{\prod_{\beta=1}^{N_{a}} 2 i \sinh \frac{a_{j}-b_{\beta}+i \varepsilon \lambda}{2}}{\prod_{l \in \vec{\gamma}} 2 i \sinh \frac{a_{j}-a_{l}+i \varepsilon\left(\lambda-\mu_{l}\right)}{2} \prod_{\alpha \notin \vec{\gamma}}^{N_{f}} 2 i \sinh \frac{a_{\alpha}-a_{j}+i \varepsilon\left(\lambda-\mu_{j}\right)}{2}} .
\end{aligned}
$$

The map of parameters in $Z_{\mathrm{v}}$ and $Z_{\mathrm{av}}$ precisely agrees with our general expression (2.68). As we will see in section 3.5 , precisely the same function $Z_{\text {vortex }}$ controls the partition function on $S^{2} \times S^{1}$. Such an expression for $Z_{\text {vortex }}$ can be compared with [52]. ${ }^{13}$

\subsection{Comparison with the two-dimensional vortex partition function}

Let us check that by taking the limit of small equivariant parameter and scaling at the same time all other parameters in the same way, the $3 \mathrm{~d}$ vortex partition function $(2.77)$ reduces to the $2 \mathrm{~d}$ vortex partition function. After a redefinition $\varepsilon \rightarrow-\varepsilon$, we take a limit $\varepsilon \rightarrow 0$ in (2.77) keeping the ratios $a_{\alpha} / \varepsilon$ and $b_{\beta} / \varepsilon$ finite; we also send the CS level $k \rightarrow 0$, that corresponds to $Q_{j} \rightarrow 1$ and $L_{j} \rightarrow z$. We get

$$
Z_{\text {vortex }}^{(\vec{\gamma})} \rightarrow \sum_{\vec{\mu} \in \mathbb{Z}_{\geq 0}^{N}} \frac{z^{|\vec{\mu}|}}{(-\varepsilon)^{\left(N_{f}-N_{a}\right)|\vec{\mu}|}} \prod_{j \in \vec{\gamma}} \frac{\prod_{\beta=1}^{N_{a}}\left(\frac{i a_{j}-i b_{\beta}}{\varepsilon}\right)_{\mu_{j}}}{\prod_{l \in \vec{\gamma}}\left(\frac{i a_{j}-i a_{l}}{\varepsilon}-\mu_{l}\right)_{\mu_{j}} \prod_{\alpha \notin \vec{\gamma}}^{N_{f}}\left(\frac{i a_{\alpha}-i a_{j}}{\varepsilon}-\mu_{j}\right)_{\mu_{j}}} .
$$

Here $|\vec{\mu}|=\sum_{j} \mu_{j}$ and we used the Pochhammer symbol $(a)_{n}=\prod_{k=0}^{n-1}(a+k)$. This expression is precisely the standard two-dimensional vortex partition function in $\Omega$-background, see e.g. [4].

\section{$3 \quad$ Higgs branch localization on $S^{2} \times S^{1}$}

We will now move to the similar study of Higgs branch localization for $\mathcal{N}=2$ theories on $S^{2} \times S^{1}$, whose path integral computes the three-dimensional supersymmetric index [54]. Localization on the Coulomb branch for $\mathcal{N}=6$ Chern-Simons-matter theories was first performed in [15], and later generalized to $\mathcal{N}=2$ theories in [16] (see also [55] for a further generalization in which magnetic fluxes for global symmetries are introduced). It was later pointed out in [24] (see also [56]) that in the presence of non-trivial magnetic fluxes, the angular momentum of fields can be shifted by half-integer amounts, thus correcting the naive fermion number: such a different weighing of the magnetic sectors helps to verify various expected dualities.

The expression that results from Coulomb branch localization is a matrix integral over the holonomy of the gauge field. As in the previous section, we will perform an alternative

\footnotetext{
${ }^{13}$ See also [53].
} 
Higgs branch localization, in which the relevant BPS configurations are discrete Higgs branches accompanied by towers of vortex strings at the north and south poles of the two-sphere.

\subsection{Killing spinors on $S^{2} \times S^{1}$, supersymmetric index and deformed background}

Supersymmetric theories on three-manifolds, among which $S^{2} \times S^{1}$, have been studied in $[34,35]$ considering the rigid limit of supergravity. In this approach, the auxiliary fields of the supergravity multiplet are treated as arbitrary background fields and SUSY backgrounds are found by setting to zero the gravitino variations; in the presence of flavor symmetries, one similarly sets to zero the external gaugino variations.

Here we will take a different approach: we will first recall the Killing spinor solutions on $S^{2} \times \mathbb{R}$, and then compactify $\mathbb{R}$ to $S^{1}$ with some twisted boundary conditions: the supersymmetric index with respect to the supercharges described by the Killing spinors indeed imposes twisted boundary conditions. In a path integral computation, however, the twisted boundary conditions are most conveniently described by turning on background fields for the charges appearing in the index formula, which finally leads to the desired theory on a deformed background.

We take the metric

$$
d s^{2}=r^{2}\left(d \theta^{2}+\sin ^{2} \theta d \varphi^{2}\right)+d \tau^{2},
$$

with vielbein $e^{1}=r d \theta, e^{2}=r \sin \theta d \varphi, e^{3}=d \tau$, and set the background $\mathrm{U}(1)_{R}$ field $V_{\mu}$ to zero. The spin connection is $\omega^{12}=-\cos \theta d \varphi$. Consider the Killing spinor equation

$$
D_{\mu} \epsilon=\gamma_{\mu} \hat{\varepsilon}
$$

where $D_{\mu}=\partial_{\mu}+\frac{1}{4} \omega_{\mu}^{a b} \gamma_{a b}$. Following [16] we consider the factorized ansatz $\epsilon_{ \pm}=$ $f(\tau) \epsilon_{ \pm}^{S^{2}}(\theta, \varphi)$, where the $2 \mathrm{~d}$ spinor satisfies $D_{\hat{\mu}} \epsilon_{ \pm}^{S^{2}}= \pm \frac{1}{2 r} \gamma_{\hat{\mu}} \gamma^{3} \epsilon_{ \pm}^{S^{2}}$ with $\hat{\mu}=\theta, \varphi$. Plugging in (3.2) gives

$$
\epsilon_{ \pm}=e^{ \pm \tau / 2 r} \epsilon_{ \pm}^{S^{2}}(\theta, \varphi), \quad D_{\mu} \epsilon_{ \pm}= \pm \frac{1}{2 r} \gamma_{\mu} \gamma^{3} \epsilon_{ \pm}
$$

Notice that the spinors are not periodic on $S^{1}$ and twisted boundary conditions will be needed. On the sphere $S^{2}$ there are four Killing spinors; then we can write the $S^{2} \times \mathbb{R}$ spinors in a compact form as

$$
\epsilon_{ \pm}=e^{ \pm \tau / 2 r} \exp \left(\mp \frac{i \theta}{2} \gamma_{2}\right) \exp \left(\frac{i \varphi}{2} \gamma_{3}\right) \epsilon_{0}
$$

where $\epsilon_{0}=\left(\begin{array}{l}C_{1} \\ C_{2}\end{array}\right)$ is constant.

Killing spinors for supersymmetric index. We will choose the spinor $\epsilon$ to be "positive" and with $\epsilon_{0}=\left(\begin{array}{l}1 \\ 0\end{array}\right)$ (so that $\left.\gamma_{3} \epsilon_{0}=\epsilon_{0}\right)$ and $\bar{\epsilon}$ to be "negative" and with $\bar{\epsilon}_{0}=\left(\begin{array}{l}0 \\ 1\end{array}\right)$ (so that $\left.\gamma_{3} \bar{\epsilon}_{0}=-\bar{\epsilon}_{0}\right)$ :

$$
\epsilon=e^{\tau / 2 r} e^{i \frac{\varphi}{2}}\left(\begin{array}{c}
\cos \theta / 2 \\
\sin \theta / 2
\end{array}\right), \quad \bar{\epsilon}=e^{-\tau / 2 r} e^{-i \frac{\varphi}{2}}\left(\begin{array}{c}
\sin \theta / 2 \\
\cos \theta / 2
\end{array}\right) .
$$


Another useful spinor is

$$
\tilde{\epsilon}=-\bar{\epsilon}^{c}=i e^{-\tau / 2 r} e^{i \frac{\varphi}{2}}\left(\begin{array}{c}
\cos \theta / 2 \\
-\sin \theta / 2
\end{array}\right)
$$

which is also a "negative" Killing spinor. We choose them of opposite positivity so that bilinears be independent of $\tau$; this also guarantees that there are no dilations in the algebra $(\rho=0)$. With these choices, the Killing vector and the functions appearing in the algebra are

$$
v^{a}=\bar{\epsilon} \gamma^{a} \epsilon=-\tilde{\epsilon}^{\dagger} \gamma^{a} \epsilon=(0, \sin \theta, i), \quad \bar{\epsilon} \epsilon=-\tilde{\epsilon}^{\dagger} \epsilon=i \cos \theta, \quad \alpha=\frac{1}{r} \bar{\epsilon} \gamma^{3} \epsilon=\frac{i}{r}
$$

We also have

$$
v=i\left(\bar{\epsilon} \gamma^{\mu} \epsilon\right) \partial_{\mu}=\frac{i}{r} \partial_{\varphi}-\partial_{\tau} .
$$

On the other hand $\epsilon^{\dagger} \epsilon=e^{\tau / r}$ and $\tilde{\epsilon}^{\dagger} \tilde{\epsilon}=e^{-\tau / r}$, as required by the dimension $\Delta$ (see below). The quantum numbers of the spinors are:

\begin{tabular}{cccc}
\hline Spinor & $\Delta$ & $j_{3}$ & $R$ \\
\hline$\epsilon$ & $-1 / 2$ & $1 / 2$ & -1 \\
$\bar{\epsilon}$ & $1 / 2$ & $-1 / 2$ & 1 \\
\hline
\end{tabular}

obtained by acting with the operators $\Delta$ and $j_{3}$ as defined below; the R-charge follows from the supersymmetry variations. We also have

$$
\left[\delta_{\epsilon}, \delta_{\bar{\epsilon}}\right]=\frac{1}{r}\left((\underbrace{-r \mathcal{L}_{\partial_{\tau}}^{A}}_{=\Delta})-(\underbrace{-i \mathcal{L}_{\partial_{\varphi}}^{A}+r \cos \theta \sigma}_{=j_{3}})-R\right) .
$$

Supersymmetric index and deformed background. The spinors are preserved by the mutually commuting operators

$$
\Delta-j_{3}-R, \quad R+2 j_{3} .
$$

The first one is the commutator $\left[\delta_{\epsilon}, \delta_{\bar{\epsilon}}\right]$. We will compute the index

$$
I\left(x, \zeta_{i}\right)=\operatorname{Tr}(-1)^{2 j_{3}} e^{-\beta\left(\Delta-j_{3}-R\right)} e^{-\xi\left(R+2 j_{3}\right)} e^{i \sum_{j} \mathfrak{z}_{j} F_{j}} \quad \text { with } \quad x=e^{-\xi}, \quad \zeta_{j}=e^{i \mathfrak{z}_{j}} .
$$

Here $F_{j}$ are the Cartan generators of the flavor symmetries and the circumference of $S^{1}$ is $\beta r$. To correctly describe the fermion number in the presence of magnetic fluxes, we have used $2 j_{3}[24,56]$. Notice that convergence of the trace requires $|x|<1$. For each Cartan generator of the flavor symmetry, besides the chemical potential $\zeta_{j}$ one could also turn on a fixed background flux on $S^{2}$ [55]: the only example we will consider in this paper is a flux for the topological symmetry $\mathrm{U}(1)_{J}$.

In the path integral formulation on $S^{2} \times S^{1}$, the index is described by the twisted periodicity conditions

$$
\Phi(\tau+\beta r)=e^{\beta\left(-j_{3}-R\right)} e^{\xi\left(R+2 j_{3}\right)} e^{-i \sum_{j} \mathfrak{z}_{j} F_{j}} \Phi(\tau) .
$$


These are also the boundary conditions satisfied by the spinors (with $F_{j}=0$ ). By the field redefinition $\tilde{\Phi} \equiv e^{-\frac{\tau}{\beta r}\left(\beta\left(-j_{3}-R\right)+\xi\left(R+2 j_{3}\right)-i \sum_{j} j_{j} F_{j}\right)} \Phi$, one can make the fields periodic again; such a redefinition is in fact a gauge transformation, indeed one can alternatively turn on background flat connections on $S^{1}$ :

$$
V_{\mu}=\left(0,0,-\frac{i}{r}+\frac{i \xi}{\beta r}\right), \quad \quad \tilde{V}_{\mu}^{(j)}=\left(0,0, \frac{\mathfrak{z} j}{\beta r}\right)
$$

for the R- and flavor symmetries respectively. The twist by the rotational symmetry imposes the identification $(\tau, \varphi) \sim(\tau+\beta r, \varphi-i(\beta-2 \xi))$. Introducing coordinates $\hat{\tau}=\tau$ and $\hat{\varphi}=\varphi+\frac{i(\beta-2 \xi)}{\beta r} \tau$, the identification becomes $(\hat{\tau}, \hat{\varphi}) \sim(\hat{\tau}+\beta r, \hat{\varphi})$. In hatted coordinates the metric $(3.1)$ is

$$
d s^{2}=r^{2} d \theta^{2}+r^{2} \sin ^{2} \theta\left[d \hat{\varphi}-\frac{i}{r}\left(1-\frac{2 \xi}{\beta}\right) d \hat{\tau}\right]^{2}+d \hat{\tau}^{2}
$$

which is complex. This metric can also be rewritten as

$$
d s^{2}=r^{2} d \theta^{2}+\frac{r^{2} \sin ^{2} \theta}{1-\left(1-\frac{2 \xi}{\beta}\right)^{2} \sin ^{2} \theta} d \hat{\varphi}^{2}+\left(1-\left(1-\frac{2 \xi}{\beta}\right)^{2} \sin ^{2} \theta\right)\left(d \hat{\tau}-\frac{i r\left(1-\frac{2 \xi}{\beta}\right) \sin ^{2} \theta}{1-\left(1-\frac{2 \xi}{\beta}\right)^{2} \sin ^{2} \theta} d \hat{\varphi}\right)^{2}
$$

which is a circle-fibration over a squashed two-sphere.

The index is thus computed by the partition function on a deformed background. A vielbein for (3.13) is $e^{1}=r d \theta, e^{2}=r \sin \theta\left(d \hat{\varphi}-\frac{i}{r}\left(1-\frac{2 \xi}{\beta}\right) d \hat{\tau}\right), e^{3}=d \hat{\tau}$, and the frame vectors are $e_{1}=\frac{1}{r} \partial_{\theta}, e_{2}=\frac{1}{r \sin \theta} \partial_{\hat{\varphi}}, e_{3}=\partial_{\hat{\tau}}+\frac{i}{r}\left(1-\frac{2 \xi}{\beta}\right) \partial_{\hat{\varphi}}$. The non-vanishing component of the spin connection is $\omega^{12}=-\cos \theta\left(d \hat{\varphi}-\frac{i}{r}\left(1-\frac{2 \xi}{\beta}\right) d \hat{\tau}\right)$. The Killing spinors corresponding to $(3.5)$ are

$$
\epsilon=e^{i \hat{\varphi} / 2}\left(\begin{array}{c}
\cos \theta / 2 \\
\sin \theta / 2
\end{array}\right), \quad \bar{\epsilon}=e^{-i \hat{\varphi} / 2}\left(\begin{array}{c}
\sin \theta / 2 \\
\cos \theta / 2
\end{array}\right) .
$$

They satisfy $D_{\mu} \varepsilon=\frac{1}{2 r} \gamma_{\mu} \gamma^{3} \varepsilon$ and $D_{\mu} \bar{\varepsilon}=-\frac{1}{2 r} \gamma_{\mu} \gamma^{3} \bar{\varepsilon}$, where $D_{\mu}=\partial_{\mu}+\frac{1}{4} \omega_{\mu}^{a b} \gamma_{a b}-i V_{\mu}-$ $i \sum_{j} \tilde{V}_{\mu}^{(j)}$, and $\tilde{\epsilon}=-\bar{\epsilon}^{c}$. The Killing vector and the functions appearing in the algebra are

$$
v^{a}=\bar{\epsilon} \gamma^{a} \epsilon=(0, \sin \theta, i), \quad \bar{\epsilon} \epsilon=i \cos \theta, \quad \alpha=\frac{i \xi}{\beta r}, \quad v^{\mu}=i \bar{\epsilon} \gamma^{\mu} \epsilon=\left(0, \frac{2 i \xi}{\beta r},-1\right) .
$$

We thus find

$$
\left[\delta_{\epsilon}, \delta_{\bar{\epsilon}}\right]=-\mathcal{L}_{\partial_{\hat{\tau}}}^{A}+\frac{2 i \xi}{\beta r} \mathcal{L}_{\partial_{\hat{\varphi}}}^{A}-\cos \theta \sigma-\frac{\xi}{\beta r} R+i \sum_{j} \frac{\mathfrak{z}_{j}}{\beta r} F_{j}
$$

From standard arguments, it is known that the index is independent of the parameter $\beta$. A significant simplification takes place by setting $\beta=2 \xi$, since the rotational symmetry charge disappears from the trace (3.10), and the complex metric (3.13) becomes the real metric on the product space $S^{2} \times S^{1}$. Henceforth, we make this choice for the immaterial parameter $\beta$ and we further omit the hats. 


\subsection{The BPS equations}

We will now proceed to derive the BPS equations. We define the quantities

$$
Y_{a}=W_{a}+\delta_{a 3} \frac{\sigma}{r}
$$

where $W_{a}$ was defined in (2.13). Using the explicit expressions for the Killing spinors (3.15), the BPS equations from the gaugino variations (B.14) can be written as

$$
\begin{array}{ll}
0=\left(Y_{3}+i D\right) \cos \frac{\theta}{2}+\left(D_{1} \sigma-i Y_{2}\right) \sin \frac{\theta}{2}, & 0=D_{3} \sigma \cos \frac{\theta}{2}+\left(Y_{1}-i D_{2} \sigma\right) \sin \frac{\theta}{2} \\
0=\left(-Y_{3}+i D\right) \sin \frac{\theta}{2}+\left(D_{1} \sigma+i Y_{2}\right) \cos \frac{\theta}{2}, & 0=-D_{3} \sigma \sin \frac{\theta}{2}+\left(Y_{1}+i D_{2} \sigma\right) \cos \frac{\theta}{2} .
\end{array}
$$

The localization locus can also be obtained from the positive definite deformation action (2.16), where now the action of $\ddagger$ is defined to be

$$
\begin{aligned}
(\mathcal{Q} \lambda)^{\ddagger} & =\epsilon^{\dagger}\left(-\frac{1}{2} \gamma^{\mu \nu} F_{\mu \nu}-D-i \gamma^{\mu} D_{\mu} \sigma-\frac{i}{r} \sigma \gamma^{3}\right)=\epsilon^{\dagger}\left(-i \gamma^{r}\left(Y_{r}+D_{r} \sigma\right)-D\right) \\
\left(\mathcal{Q} \lambda^{\dagger}\right)^{\ddagger} & =\left(\frac{1}{2} \gamma^{\mu \nu} F_{\mu \nu}+D-i \gamma^{\mu} D_{\mu} \sigma+\frac{i}{r} \sigma \gamma^{3}\right) \tilde{\epsilon}=\left(i\left(Y_{r}-D_{r} \sigma\right) \gamma^{r}+D\right) \tilde{\epsilon} .
\end{aligned}
$$

One then obtains $\mathcal{L}_{\mathrm{YM}}^{\mathrm{def}}=\frac{1}{2} \operatorname{Tr}\left[\left(Y_{\mu}\right)^{2}+\left(D_{\mu} \sigma\right)^{2}+D^{2}\right]$. Imposing the reality conditions, the Coulomb branch localization locus immediately follows:

$$
Y_{\mu}=0, \quad D_{\mu} \sigma=0, \quad D=0 \text {. }
$$

Note that the string-like vortices are excluded by these equations since they imply $D_{\mu} F_{12}=0$.

Higgs branch localization can be achieved by adding another $\mathcal{Q}$-exact term to the deformation action. We use the same term as in (2.19):

$$
\mathcal{L}_{\mathrm{H}}^{\mathrm{def}}=\mathcal{Q} \operatorname{Tr}\left[\frac{i\left(\epsilon^{\dagger} \lambda-\lambda^{\dagger} \tilde{\epsilon}\right) H(\phi)}{2}\right]
$$

whose bosonic piece is

$$
\left.\mathcal{L}_{\mathrm{H}}^{\text {def }}\right|_{\text {bos }}=-\operatorname{Tr}\left[\left(\sin \theta\left(D_{1} \sigma\right)+\cos \theta Y_{3}+i D\right) H(\phi)\right] .
$$

The Gaussian path integral over $D$ imposes

$$
D=i H(\phi) .
$$

Then one is left with

$$
\begin{aligned}
\mathcal{L}_{\mathrm{YM}}^{\text {def }}+\left.\mathcal{L}_{\mathrm{H}}^{\text {def }}\right|_{D, \text { bos }}= & \frac{1}{2} \operatorname{Tr}\left[\left(D_{1} \sigma \cos \theta-Y_{3} \sin \theta\right)^{2}+\left(H(\phi)-D_{1} \sigma \sin \theta-Y_{3} \cos \theta\right)^{2}\right. \\
& \left.+\left(Y_{2}\right)^{2}+\left(D_{2} \sigma\right)^{2}+\left(Y_{1}\right)^{2}+\left(D_{3} \sigma\right)^{2}\right]
\end{aligned}
$$


which is a sum of squares. The BPS equations are then

$$
\begin{array}{ll}
0=D_{1} \sigma \cos \theta-\left(F_{12}+\frac{\sigma}{r}\right) \sin \theta, & 0=D_{2} \sigma=D_{3} \sigma \\
0=H(\phi)-D_{1} \sigma \sin \theta-\left(F_{12}+\frac{\sigma}{r}\right) \cos \theta, & 0=F_{13}=F_{23} .
\end{array}
$$

Consider now the chiral multiplets, transforming in some representation $\mathfrak{R}=\bigoplus_{j} \mathcal{R}_{j}$ of the gauge and flavor group, where $\mathcal{R}_{j}$ are irreducible gauge representations. Imposing the reality conditions $\bar{\phi}^{\dagger}=\phi, \bar{F}^{\dagger}=F$ and $\sigma^{\dagger}=\sigma$, one finds the BPS equations

$$
\begin{array}{ll}
0=\sin \frac{\theta}{2} D_{+} \phi+\cos \frac{\theta}{2}\left(\frac{D_{3}+D_{3}^{\dagger}}{2} \phi+\frac{q}{r} \phi+\sigma \phi\right), & 0=\left(D_{3}-D_{3}^{\dagger}\right) \phi \\
0=\cos \frac{\theta}{2} D_{-} \phi-\sin \frac{\theta}{2}\left(\frac{D_{3}+D_{3}^{\dagger}}{2} \phi+\frac{q}{r} \phi-\sigma \phi\right), & 0=F,
\end{array}
$$

where $D_{ \pm} \equiv D_{1} \mp i D_{2}$ and $D_{3} \phi=D_{\tau} \phi=\left(\partial_{\tau}-i \frac{a}{2 \xi r}-\frac{1}{2 r} q-i \frac{\mathfrak{s}}{2 \xi r}\right) \phi$.

As before, these equations can be obtained from the canonical deformation action $\mathcal{L}_{\text {mat }}^{\text {def }}$. Its bosonic part reads

$$
\begin{aligned}
\left.\mathcal{L}_{\text {mat }}^{\text {def }}\right|_{\text {bos }}= & \frac{1}{2}|F|^{2}+\frac{1}{8}\left|D_{3} \phi-D_{3}^{\dagger} \phi\right|^{2}+\frac{1}{2}\left|\sin \frac{\theta}{2} D_{+} \phi+\cos \frac{\theta}{2}\left(\frac{D_{3}+D_{3}^{\dagger}}{2} \phi+\frac{q}{r} \phi+\sigma \phi\right)\right|^{2} \\
& +\frac{1}{2}\left|\cos \frac{\theta}{2} D_{-} \phi-\sin \frac{\theta}{2}\left(\frac{D_{3}+D_{3}^{\dagger}}{2} \phi+\frac{q}{r} \phi-\sigma \phi\right)\right|^{2}
\end{aligned}
$$

\subsection{BPS solutions: Coulomb, Higgs and vortices}

We will now present the BPS solutions to the equations (3.21), (3.25) and (3.26). First, let us recall the solutions for the standard choice $H(\phi)=0$.

Coulomb-like solutions. Consider (3.21) and (3.26). They allow for a field strength

$$
F=\frac{\mathfrak{m}}{2} \sin \theta d \theta \wedge d \varphi
$$

where $\mathfrak{m}$ can be diagonalized to lie in the Cartan subalgebra and it takes values in the coweight lattice of the gauge group $G$ (it is GNO quantized). The gauge field can be written as

$$
A=\frac{\mathfrak{m}}{2}(\kappa-\cos \theta) d \varphi+\frac{a}{2 \xi r} d \tau,
$$

where in this section $\kappa=1(\kappa=-1)$ on the patch excluding the south (north) pole. We have also included a holonomy $a$, with $[a, \mathfrak{m}]=0$, around the temporal circle. The BPS equations fix $\sigma=-\mathfrak{m} / 2 r$ and $D=0$, which is the localization locus of [16].

Let us now analyze the BPS equations for a chiral multiplet in gauge representation $\mathcal{R}$, assuming that its R-charge $q$ is positive, and show that the only smooth solution is 
$\phi=0$. First, we decompose $\phi$ in Fourier modes recalling that, in the presence of nontrivial flux on $S^{2}, \phi$ is a section of a non-trivial bundle and should be expanded in monopole harmonics [57]:

$$
\phi(\tau, \theta, \varphi)=\sum_{p, l, m} c_{p, l, m} \exp \left(\frac{2 \pi i p \tau}{2 \xi r}\right) Y_{\frac{\mathfrak{m}}{2}, l, m}
$$

where the range of parameters is $p \in \mathbb{Z}, l \in \frac{|\mathfrak{m}|}{2}+\mathbb{N}$ and $m=-l,-l+1, \ldots,+l$. The third component of the angular momentum is given by the eigenvalue of ${ }^{14}$

$$
j_{3}=-i \partial_{\varphi}-\kappa \frac{\mathfrak{m}}{2}
$$

and on the monopole harmonics: $j_{3} Y_{\frac{\mathfrak{m}}{2}, l, m}=m Y_{\frac{\mathfrak{m}}{2}, l, m}$. Imposing a Hermiticity condition on the holonomy $a$, the equation $\left(D_{3}-D_{3}^{\dagger}\right) \phi=0$ corresponds to

$$
\left(\partial_{\tau}-i \frac{a}{2 \xi r}-i \frac{\mathfrak{z}}{2 \xi r}\right) \phi=0 \text {. }
$$

This implies that only those modes for which $(a-2 \pi p+\mathfrak{z}) \phi=0$ can survive. Since the time dependence is completely fixed, we can reabsorb $p$ by a large gauge transformation and set $p=0$. From the equations in the first column of (3.26), the expressions for $\sigma$ and the gauge field found above, we find $\left(j_{3}+\frac{q}{2}\right) \phi=0$ and $j_{+} \phi=0$. The first one imposes $m=-q / 2$, whereas the second one imposes that the angular momentum eigenvalue $m$ take its maximal value $+l$. For positive R-charge $q>0$, there are no solutions. For zero R-charge (then $l=m=0$ ) one finds the constant Higgs-like solution $\phi=\phi_{0}$, if $(a+\mathfrak{z}) \phi=0$.

Now let us see the new solutions with non-trivial $H(\phi)$. We integrate $D$ out first, i.e. we set $D=i H(\phi)$, solve (3.25) and (3.26), and take all vanishing R-charges $q=0$ (arbitrary R-charges can be recovered by analytic continuation of the result by complexifying flavor fugacities). We take exactly the same deformation function $H(\phi)$ as in (2.35). We find the following classes of solutions.

Deformed Coulomb branch. It is characterized by $\phi=0$, and (in complete analogy with [4]) can be completed to

$$
F_{13}=F_{23}=0, \quad F_{12}=2 \zeta \cos \theta+\frac{\mathfrak{m}}{2 r^{2}}, \quad \sigma=-r \zeta \cos \theta-\frac{\mathfrak{m}}{2 r} .
$$

We thus have $F_{\theta \varphi}=r^{2} \sin \theta\left(2 \zeta \cos \theta+\mathfrak{m} / 2 r^{2}\right)$. The corresponding gauge field can be written as

$$
A=\left(r^{2} \zeta \sin ^{2} \theta+\frac{\mathfrak{m}}{2}(\kappa-\cos \theta)\right) d \varphi+\frac{a}{2 \xi r} d \tau
$$

\footnotetext{
${ }^{14}$ From [57], the gauge invariant angular momentum operator on $\mathbb{R}^{3}$ in a monopole background $\mathfrak{m}$ is given by $\vec{L}=\vec{r} \times(-i \vec{D})-\hat{r} \mathfrak{m} / 2$, where $\hat{r}$ is a unit vector along the $\vec{r}$ direction. In particular the third component is

$$
j_{3}=-i D_{\varphi}-\mathfrak{m} / 2 \cos \theta=-i \partial_{\varphi}-\kappa \frac{\mathfrak{m}}{2} .
$$

This result is directly applicable to $S^{2}$. For later reference, we also write the operators $j_{+}$and $j_{-}$:

$$
j_{ \pm}=e^{ \pm i \varphi}\left( \pm \partial_{\theta}+i \cot \theta D_{\varphi}-\frac{\mathfrak{m}}{2} \sin \theta\right)=e^{ \pm i \varphi}\left( \pm \partial_{\theta}+i \cot \theta \partial_{\varphi}+\frac{\mathfrak{m}}{2} \kappa \cot \theta-\frac{\mathfrak{m}}{2} \frac{1}{\sin \theta}\right)
$$


Higgs-like solutions. They are characterized by $F_{\mu \nu}=0, \sigma=0$ and a constant profile $\phi$ for the matter fields that solves the D-term equations

$$
H(\phi)=0, \quad(a+\mathfrak{z}) \phi=0 .
$$

The solutions to these algebraic equations are analogous to the Higgs-like solutions of section 2.3. We will be mainly interested in gauge groups and matter representations such that, for $\zeta_{a}$ in a suitable range, all VEVs $\phi$ completely break the gauge group.

Vortices. Each Higgs-like solution is accompanied by a tower of vortex-string solutions with arbitrary numbers of vortices at the north and at the south circles. To see this, we expand the BPS equations around $\theta=0$ and $\theta=\pi$.

The $S^{2} \times S^{1}$ metric (3.1) in the $\theta \rightarrow 0$ limit becomes $d s^{2}=d R^{2}+R^{2} d \varphi^{2}+d \tau^{2}$, where $R \equiv r \theta$, which is the metric of $\mathbb{R}^{2} \times S^{1}$. The equations (3.25) become, to linear order in $R$ :

$$
\begin{aligned}
0 & =D_{R} \sigma-\frac{1}{r} F_{R \varphi}, & 0 & =D_{\varphi} \sigma=D_{\tau} \sigma \\
0 & =H(\phi)-\frac{1}{R} F_{R \varphi}-D_{R}\left(\frac{\sigma R}{r}\right), & 0 & =F_{\varphi \tau}=F_{R \tau},
\end{aligned}
$$

whereas the equations for the chiral fields (3.26) become

$$
\begin{aligned}
0 & =\left(D_{R}+\frac{i}{R} D_{\varphi}+\frac{R}{r} \sigma\right) \phi, & 0 & =\left(D_{3}-D_{3}^{\dagger}\right) \phi \\
0 & =\left(-\frac{i}{r} D_{\varphi}+\sigma+\frac{D_{3}+D_{3}^{\dagger}}{2}\right) \phi, & 0 & =F .
\end{aligned}
$$

Let us qualitatively describe the solutions for a $U(1)$ theory with a single chiral field of charge 1. Working in the gauge $A_{\theta}=0,(3.25)$ implies that $\partial_{\theta}\left(r \sigma \cos \theta-A_{\varphi}\right)=0$ exactly. We write $r \sigma \cos \theta=A_{\varphi}-n$, for some integration constant $n$, so it is sufficient to specify the behavior of $\phi$ and $A_{\varphi}$. Far from the core (the length scale is set by $\sqrt{\zeta^{-1}}$ ) one finds

$$
\phi \simeq \sqrt{\zeta} e^{i n \varphi}, \quad A_{\varphi} \simeq n,
$$

and Stokes' theorem implies that $\frac{1}{2 \pi} \int F=n$, which is the vortex number. Close to the core:

$$
\phi \simeq B\left(R e^{i \varphi}\right)^{n}, \quad A_{\varphi} \simeq 0+\mathcal{O}\left(e^{-\frac{R^{2}}{2 r^{2}}}\right),
$$

and in particular $n \geq 0$. A similar analysis can be performed around the south pole in the coordinate $\tilde{R}=r(\pi-\theta)$. This time we write $r \sigma \cos \theta=A_{\varphi}-m$. Then $A_{\varphi} \rightarrow 0$ near the core and $A_{\varphi} \rightarrow m$, which we identify with the vortex number, far from the core. We also find $|\phi| \rightarrow B^{\prime} \tilde{R}^{m}$ near the core, while it sits in the vacuum far from it: $|\phi|^{2} \rightarrow \zeta$. The vortex configurations we wrote around the north and south poles are connected by a gauge transformation on the equator: $\phi^{N}=e^{i(n-m) \varphi} \phi^{S}$ and $A_{\varphi}^{N}-A_{\varphi}^{S}=n-m$.

For finite values of $\zeta$, we can derive a bound on the allowed vortex numbers. From (3.25) one deduces $H(\phi) r \sin \theta=\partial_{\theta} \sigma$, which results in the inequality

$$
r \sigma(\pi)-r \sigma(0)=\frac{1}{2 \pi} \int H(\phi) d \operatorname{vol}\left(S^{2}\right) \leq \zeta \frac{\operatorname{vol}\left(S^{2}\right)}{2 \pi},
$$


which upon plugging in the values of $\sigma$ found above leads to the bound

$$
m+n \leq \zeta \frac{\operatorname{vol}\left(S^{2}\right)}{2 \pi} .
$$

As in section 2.3, we conclude that for finite values of $\zeta$ there is a finite number of vortex/antivortex solutions on $S^{2}$. When the bound is saturated, the chiral field $\phi$ actually vanishes and the gauge field is as in the deformed Coulomb branch described above. We thus get a similar picture of the structure of solutions as in section 2.3.

\subsection{Computation of the index}

We will now evaluate the classical action and the one-loop determinants of quadratic fluctuations, and then sum/integrate over the space of BPS configurations.

\subsubsection{One-loop determinants from the index theorem}

As in section 2.4.1, we compute the one-loop determinants on non-trivial backgrounds with the equivariant index theorem, following [46]. The localizing supercharge squares to

$$
\mathcal{Q}^{2}=-\mathcal{L}_{\partial_{\tau}}^{A}+\frac{i}{r} \mathcal{L}_{\partial_{\varphi}}^{A}-\cos \theta \sigma-\frac{1}{2 r} R+i \sum_{j} \frac{\mathfrak{z}_{j}}{2 \xi r} F_{j}
$$

The action of $\mathcal{Q}^{2}$ on the worldvolume consists of a free rotation along $S^{1}$ generated by $\mathcal{L}_{\partial_{\tau}}$ and a rotation of $S^{2}$ generated by $\mathcal{L}_{\partial_{\varphi}}$ with fixed points at the north and south poles. The equivariant parameters for the $\mathrm{U}(1)_{\partial_{\varphi}} \times \mathrm{U}(1)_{R} \times \mathrm{U}(1)_{\text {flavor }}^{F} \times G$ are given by $\varepsilon=\frac{i}{r}$, $\hat{\varepsilon}=-\frac{1}{2 r}, \check{\varepsilon}_{j}=i \frac{\mathfrak{z} j}{2 \xi r}$ and $\hat{a}=i A_{\tau}+\frac{1}{r} A_{\varphi}-\cos \theta \sigma$. In appendix $\mathrm{C}$ we compute the one-loop determinants in our conventions. For a chiral multiplet in gauge representation $\mathcal{R}$ we have

$$
Z_{1-\text { loop }}^{\text {chiral }} "=" \prod_{w \in \mathcal{R}} \prod_{n \in \mathbb{Z}} \prod_{k \geq 0} \frac{i \pi n-(k+1) \xi+\xi \frac{q}{2}-\xi r w\left(\hat{a}_{S}\right)-\frac{i}{2} \sum \mathfrak{z}_{j} F_{j}}{i \pi n+k \xi+\xi \frac{q}{2}-\xi r w\left(\hat{a}_{N}\right)-\frac{i}{2} \sum \mathfrak{z}_{j} F_{j}}
$$

which requires regularization. For the gauge multiplet one has

$$
Z_{1-\text { loop }}^{\mathrm{vec}}=\prod_{\alpha>0} 2 \sinh \left(\xi r \alpha\left(\hat{a}_{N}\right)\right) 2 \sinh \left(-\xi r \alpha\left(\hat{a}_{S}\right)\right) .
$$

\subsubsection{Coulomb branch}

Coulomb branch localization for the $3 \mathrm{~d}$ index was first performed in [15] for $\mathcal{N}=6$ ChernSimons-matter theories, and later generalized to $\mathcal{N}=2$ theories in [16]. A subtlety involving the fermion number was pointed out in [24] (see also [56]), and was later confirmed in [46] by computing the one-loop determinants with the index theorem. Let us quickly review these results. The Chern-Simons action evaluated on the Coulomb branch configurations gives ${ }^{15}$

$$
S_{\mathrm{cl}}^{\mathrm{CS}}=-\frac{i}{4 \pi} \int \operatorname{Tr}_{\mathrm{CS}} A \wedge F=-i \operatorname{Tr}_{\mathrm{CS}} a \mathfrak{m} .
$$

\footnotetext{
${ }^{15}$ We recall that in order to correctly evaluate the CS action $\int A \wedge F$, one should construct an extension $\tilde{F}$ of the gauge bundle to $S^{2} \times D_{2}$ (where the second factor is a disk) and integrate $\int \tilde{F} \wedge \tilde{F}$.
} 
Due to the modified fermion number, an extra phase $(-1)^{\operatorname{Tr}_{C S} \mathfrak{m}}$ needs to be taken into account [56].

To each Abelian factor (with field strength $F$ ) in the gauge group is associated a topological symmetry $\mathrm{U}(1)_{J}$, whose current is $J=* F$. Coupling $\mathrm{U}(1)_{J}$ to an external vector multiplet with bosonic components $\left(A_{\mathrm{BG}}, \sigma_{\mathrm{BG}}, D_{\mathrm{BG}}\right)$ is equivalent to introducing a mixed supersymmetric Chern-Simons term, whose bosonic part is

$$
\left.S_{J}\right|_{\text {bos }}=\frac{i}{2 \pi} \int \operatorname{Tr}\left(A_{\mathrm{BG}} \wedge F+\sigma D_{\mathrm{BG}}+\sigma_{\mathrm{BG}} D\right) .
$$

An expectation value for $\sigma_{\mathrm{BG}}$ would correspond to an FI term. In this section, though, we will be interested in turning on a holonomy $b$ and a flux $\mathfrak{n}$. Notice that this is indeed an example of an external flux for a flavor symmetry, in the spirit of [55]. Evaluation on the Coulomb branch BPS configurations yields

$$
S_{J}=i \operatorname{Tr}(a \mathfrak{n}+b \mathfrak{m}) .
$$

We will introduce the topological fugacity $w=e^{-i b}$. Also in this case extra signs are required: this can be done by taking the index not to be a function of $w$, but rather of $(-1)^{\mathfrak{n}} w$. Such dependence will always be understood.

The gauge equivariant parameter is $\hat{a}=\frac{i a}{2 \xi r}+\frac{\kappa}{2 r} \mathfrak{m}$, where $\kappa=1(-1)$ on the northern (southern) patch, as in (3.29). The chiral one-loop determinant then simplifies and, after regularization, becomes

$$
Z_{1-\text { loop }}^{\text {chiral }}=\prod_{w \in \mathcal{R}}\left(x^{1-q} e^{-i w(a)} \zeta^{-F}\right)^{-w(\mathfrak{m}) / 2} \frac{\left(x^{2-q-w(\mathfrak{m})} e^{-i w(a)} \zeta^{-F} ; x^{2}\right)_{\infty}}{\left(x^{q-w(\mathfrak{m})} e^{i w(a)} \zeta^{F} ; x^{2}\right)_{\infty}},
$$

where $(a ; q)_{\infty} \equiv \prod_{k=0}^{\infty}\left(1-a q^{k}\right)$ is the $q$-Pochhammer symbol, we defined $x=e^{-\xi}$ and $\zeta_{j}=e^{i_{\mathfrak{z} j}}$, we used the short-hand notation $\zeta^{F}=\prod_{i} \zeta_{i}^{F_{i}}$, and $q$ is the R-charge. The regularization is similar to [15] (see also [46]). The expression above includes all the correct extra signs. The vector one-loop determinant becomes

$Z_{1-\text { loop }}^{\mathrm{vec}}=\prod_{\alpha>0} 4 \sinh \left(\frac{1}{2} \alpha(i a+\xi \mathfrak{m})\right) \sinh \left(-\frac{1}{2} \alpha(i a-\xi \mathfrak{m})\right)=\prod_{\alpha \in G} x^{-\frac{1}{2}|\alpha(\mathfrak{m})|}\left(1-x^{|\alpha(\mathfrak{m})|} e^{i \alpha(a)}\right)$.

The index is thus computed by the matrix integral:

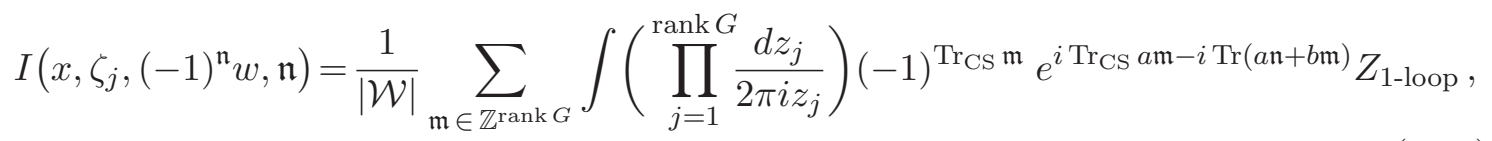

where $|\mathcal{W}|$ is the order of the Weyl group, $z_{j}=e^{i a_{j}}$ is the gauge fugacity and the integration contour is counterclockwise along the unit circle.

\subsubsection{Deformed Coulomb branch}

The full Chern Simons action (B.16) and the mixed CS term (3.48) evaluated on the deformed Coulomb branch read

$$
S_{\mathrm{cl}}^{\mathrm{CS}}=-i \operatorname{Tr}_{\mathrm{CS}}\left(\left(a-2 i r^{2} \xi \zeta\right) \mathfrak{m}\right), \quad S_{J}=\operatorname{Tr}\left(\left(a-2 i r^{2} \xi \zeta\right) \mathfrak{n}+b \mathfrak{m}\right),
$$


where, in this subsection, $\zeta$ refers to the deformation parameter (2.36). We also need to include the phase $(-1)^{\operatorname{Tr}_{C S} \mathfrak{m}}$. The equivariant parameter is given by

$$
\hat{a}=i \frac{a-2 i r^{2} \xi \zeta}{2 \xi r}+\frac{\kappa}{2 r} \mathfrak{m} .
$$

As in section 2.4.3, we observe that the net effect of the deformation parameter $\zeta$ is an imaginary shift of the integration variable $a \rightarrow a-2 i r^{2} \xi \zeta$, or equivalently $z \equiv e^{i a} \rightarrow$ $x^{-2 r^{2} \zeta} z$. Effectively it modifies the radius of the integration contour; since $|x|<1$, the contour grows for $\zeta>0$ and shrinks for $\zeta<0$. The effect on the integral is the same as in section 2.4.3: it remains constant, until the contour crosses some pole and the integral jumps. In view of the bound (3.43), this happens precisely when new vortex configuration become allowed, providing the missing residue.

In order to obtain an expression of $Z_{S^{2} \times S^{1}}$ purely in terms of vortices, we need to suppress the contribution from the deformed Coulomb branch. Heuristically, this can be achieved if there is no pole at the origin or infinity. For a $\mathrm{U}(N)$ theory with $N_{f}$ fundamentals and $N_{a}$ antifundamentals, there is no pole at infinity if $N_{f}>N_{a}$, thus suppression is obtained by sending $\zeta \rightarrow+\infty$; for $N_{f}<N_{a}$ there is no pole at the origin, thus suppression is obtained by sending $\zeta \rightarrow-\infty$. For $N_{f}=N_{a}$ there are poles both at the origin and at infinity, however the residue vanishes for a suitable range of parameters [31].

\subsubsection{Higgs branch and vortices}

For finite values of the deformation parameters $\zeta_{a}$, additional BPS configurations are present, namely Higgs vacua and vortex solutions, whose (anti)vortex numbers $(m, n)$ are bounded by (3.43) (or its multi-dimensional generalization). We determine here their additional contribution to the path integral, besides the deformed Coulomb branch. The discussion is similar to section 2.4.4, so we will be brief.

The classical actions can be evaluated exactly using $D=i H(\phi)$, the BPS equations (3.25), the knowledge of the flux carried by the vortices and of the corresponding values of $A_{\varphi}(\theta)$ at $\theta=0, \pi$, in a gauge $A_{\theta}=0$. Recall that the equations determine $\sigma$ exactly in terms of $A_{\varphi}$, see around (3.40). One finds

$$
S_{\mathrm{cl}}^{\mathrm{CS}}=-i \operatorname{Tr}_{\mathrm{CS}}\left((n-m) a+i \xi\left(m^{2}-n^{2}\right)\right), \quad S_{J}=i \operatorname{Tr}[\mathfrak{n}(a-i \xi(m+n))+b(n-m)],
$$

where $a$ is evaluated on the Higgs branch, $a=-\mathfrak{z}$. Again we need to include the extra phase $(-1)^{\operatorname{Tr} C S}(n-m)$. The one-loop determinants are evaluated with (3.45) and (3.46), using the equivariant parameters

$$
\hat{a}_{N}=\frac{i a+2 \xi n}{2 \xi r}, \quad \hat{a}_{S}=\frac{i a+2 \xi m}{2 \xi r}
$$

at the north and south poles, where in both cases $a$ is evaluated on its Higgs branch location $a_{H}$. The one-loop determinants for the $\operatorname{rank} G$ chiral multiplets Higgsing the gauge group should be computed with a residue prescription. Therefore, after a regularization similar 
to [46], the one-loop determinant for chiral multiplets is

$$
Z_{1 \text {-loop }}^{\text {chiral }}=\operatorname{Res}_{a \rightarrow a_{H}}\left[\prod_{w \in \Re}\left(x^{1+w(m+n)} e^{-i w(a)} \zeta^{-F(\phi)}\right)^{w(m-n) / 2} \frac{\left(x^{2+2 w(m)} e^{-i w(a)} \zeta^{-F(\phi)} ; x^{2}\right)_{\infty}}{\left(x^{-2 w(n)} e^{i w(a)} \zeta^{F(\phi)} ; x^{2}\right)_{\infty}}\right] .
$$

Here $F(\phi)$ refers to the chiral multiplets, $\zeta^{F}=\prod_{i} \zeta_{i}^{F_{i}}$ and we set the R-charges to zero. For the vector one-loop determinant we have

$$
\begin{aligned}
Z_{1-\text { loop }}^{\text {gauge }} & =\prod_{\alpha>0} 2 \sinh \left(\frac{\alpha(i a+2 \xi n)}{2}\right) 2 \sinh \left(-\frac{\alpha(i a+2 \xi m)}{2}\right) \\
& =\prod_{\alpha \in \mathfrak{g}} x^{-\frac{|\alpha(n-m)|}{2}}\left(1-x^{|\alpha(n-m)|-\alpha(n+m)} e^{i \alpha(a)}\right),
\end{aligned}
$$

evaluated on the Higgs branch location. These expressions, for the vortices that satisfy the bound (3.43), precisely reproduce the residues of the integrand in (3.52), which are the jumps of the deformed Coulomb branch contribution as the contour crosses the poles.

Vortex partition function. We will now take a suitable limit $\zeta_{a} \rightarrow \pm \infty$, in which the deformed Coulomb branch contribution is suppressed. Then the resummed contribution of all vortex strings is described by the same vortex partition function that we used on $S_{b}^{3}$.

Let us compute the partition function in the limit. First, we have a finite number of Higgs vacua. In each vacuum, the components of the holonomy $a_{\alpha}$ are fixed to some specific (real) values that are functions of the real masses. The classical actions (3.55) provide an overall classical contribution:

$$
S_{J}=i \operatorname{Tr}(\mathfrak{n} a),
$$

as well as the weighting factors for vortices and anti-vortices:

$$
\begin{aligned}
e^{-S_{\mathrm{v}}} & =\exp \left[-\xi \operatorname{Tr}_{\mathrm{CS}} m^{2}+\left(-i \operatorname{Tr}_{\mathrm{CS}} a \cdot+\operatorname{Tr}(-\xi \mathfrak{n}+i b) \cdot\right) m\right] \\
e^{-S_{\mathrm{av}}} & =\exp \left[\xi \operatorname{Tr}_{\mathrm{CS}} n^{2}+\left(i \operatorname{Tr}_{\mathrm{CS}} a \cdot+\operatorname{Tr}(-\xi \mathfrak{n}-i b) \cdot\right) n\right] .
\end{aligned}
$$

Second, the one-loop determinants for the vector multiplet and the chiral multiplets not acquiring a VEV are as in the Coulomb branch. The $\operatorname{rank} G$ chiral multiplets acquiring VEV bring a residue factor, which in this case is some phase. Finally, the vortex partition function $Z_{\text {vortex }}$ depends on equivariant parameters for rotations of $\mathbb{R}^{2}(\varepsilon)$ and flavor rotations $(g)$ : they are identified — at $\theta=0(\mathrm{~N})$ and $\theta=\pi(\mathrm{S})$ - from the $\mathrm{SU}(1 \mid 1)$ complex of the supercharge $\mathcal{Q}$ at the poles, i.e. from $\mathcal{Q}^{2}$ in (3.44). We find

$$
\varepsilon_{N}=-2 i \xi, \quad g_{N}=i\left(a+\sum_{j} \mathfrak{z}_{j} F_{j}\right), \quad \varepsilon_{S}=2 i \xi, \quad g_{S}=-i\left(a+\sum_{j} \mathfrak{z}_{j} F_{j}\right),
$$

where the minus sign in the south pole parameters with respect to the north pole ones is due to the opposite orientation.

Eventually, Higgs branch localization gives the following expression for the index:

$$
I=\sum_{\text {Higgs vacua }} e^{-i \operatorname{Tr}(\mathfrak{n} a)} Z_{1-\text { loop }}^{\prime} Z_{\mathrm{v}} Z_{\mathrm{av}} .
$$


The (anti)vortex-string contributions are expressed in terms of the $3 \mathrm{~d}$ vortex partition function:

$$
\begin{aligned}
& Z_{\mathrm{v}}=Z_{\text {vortex }}\left(e^{-\xi \operatorname{Tr}_{\mathrm{CS}} \cdot}, e^{-i \operatorname{Tr}_{\mathrm{CS}} a \cdot+\operatorname{Tr}(-\xi \mathfrak{n}+i b) \cdot},-2 i \xi, i\left(a+\sum_{j} \mathfrak{z}_{j} F_{j}\right)\right) \\
& Z_{\mathrm{av}}=Z_{\text {vortex }}\left(e^{\xi \operatorname{Tr}_{\mathrm{CS}} \cdot}, e^{i \operatorname{Tr}_{\mathrm{CS}} a \cdot+\operatorname{Tr}(-\xi \mathfrak{n}-i b) \cdot}, 2 i \xi,-i\left(a+\sum_{j} \mathfrak{z}_{j} F_{j}\right)\right) .
\end{aligned}
$$

As in section 2.4.4, the first two arguments in the vortex partition function are exponentiated linear functions on the gauge algebra, corresponding to the quadratic and linear weights for the vortex numbers. We shall give a concrete example in the next section.

\subsection{Matching with the Coulomb branch integral}

We wish to shortly review, in our conventions, that the superconformal index of a $\mathrm{U}(N)$ gauge theory with $N_{f}$ fundamentals and $N_{a}$ antifundamental can be rewritten in a form that matches with the result of Higgs branch localization, as done in [31], ${ }^{16}$ and moreover that the very same $Z_{\text {vortex }}$ as in (2.77) emerges.

Concretely,

$$
\begin{aligned}
& I^{\mathrm{U}(N), N_{f}, N_{a}}=\frac{1}{N !} \sum_{\overrightarrow{\mathfrak{m}} \in \mathbb{Z}^{N}} w^{\sum_{j} \mathfrak{m}_{j}} \oint \prod_{j=1}^{N}\left(\frac{d z_{j}}{2 \pi i z_{j}}\left(-z_{j}\right)^{k \mathfrak{m}_{j}} z_{j}^{-\mathfrak{n}}\right) \prod_{\substack{i, j=1 \\
i \neq j}}^{N} x^{-\left|\mathfrak{m}_{i}-\mathfrak{m}_{j}\right| / 2}\left(1-z_{i} z_{j}^{-1} x^{\left|\mathfrak{m}_{i}-\mathfrak{m}_{j}\right|}\right) \\
& \times \prod_{i=1}^{N} \prod_{\alpha=1}^{N_{f}}\left(x z_{i}^{-1} \zeta_{\alpha}\right)^{-\mathfrak{m}_{i} / 2} \frac{\left(z_{i}^{-1} \zeta_{\alpha} x^{-\mathfrak{m}_{i}+2} ; x^{2}\right)_{\infty}}{\left(z_{i} \zeta_{\alpha}^{-1} x^{-\mathfrak{m}_{i}} ; x^{2}\right)_{\infty}} \prod_{\beta=1}^{N_{a}}\left(x z_{i} \tilde{\zeta}_{\beta}^{-1}\right)^{\mathfrak{m}_{i} / 2} \frac{\left(z_{i} \tilde{\zeta}_{\beta}^{-1} x^{\mathfrak{m}_{i}+2} ; x^{2}\right)_{\infty}}{\left(z_{i}^{-1} \tilde{\zeta}_{\beta} x^{\mathfrak{m}_{i}} ; x^{2}\right)_{\infty}}
\end{aligned}
$$

where $z_{j}=e^{i a_{j}}$ and $w=e^{-i b}$. The flavor fugacities $\zeta_{\alpha}=e^{i \mathfrak{z} \alpha}, \tilde{\zeta}_{\beta}=e^{i \tilde{\mathfrak{z}} \beta}$ are defined up to a common rescaling, since the flavor symmetry is $\mathrm{SU}\left(N_{f}\right) \times \mathrm{SU}\left(N_{a}\right) \times \mathrm{U}(1)_{A}$. The integration contour is along the unit circle for $\left|\tilde{\zeta}_{\beta}\right|<1<\left|\zeta_{\alpha}\right|$. We also introduced the extra sign $(-1)^{k \sum \mathfrak{m}_{j}}$, as explained in section 3.4.2. Note that $k+\frac{N_{f}+N_{a}}{2}$ is integer if we impose parity anomaly cancelation: this guarantees that the integrand is a single-valued function of $z_{j}$.

For $N_{f}>N_{a}$ there is no pole at infinity. Moreover, since $\left|\tilde{\zeta}_{\beta}\right|<1<\left|\zeta_{\alpha}\right|$ and $|x|<1$, only the one-loop determinants of fundamentals have poles outside the unit circle. More precisely, the numerator of the one-loop determinants of fundamentals has zeros at $z_{j}=$ $\zeta_{\alpha_{j}} x^{-\mathfrak{m}_{j}+2 r_{j}}$, for all $r_{j} \geq 1$ and $j=1, \ldots, N$, while the denominator has zeros at $z_{j}=$ $\zeta_{\alpha_{j}} x^{\mathfrak{m}_{j}-2 r_{j}}$ for all $r_{j} \geq 0$. For $\mathfrak{m}_{j} \leq 0$ there is no superposition of zeros, while for $\mathfrak{m}_{j}>0$ there is superposition and some of them cancel. The net result is that the poles outside the unit circle are located at

$$
z_{j}=\zeta_{\gamma_{j}} x^{-\left|\mathfrak{m}_{j}\right|-2 r_{j}}, \quad r_{j} \in \mathbb{Z}_{\geq 0}, \quad \gamma_{j}=1, \ldots, N_{f}, \quad j=1, \ldots, N
$$

\footnotetext{
${ }^{16}$ See also [30], where the factorized form of the index was first observed in the $\mathrm{U}(1)$ case.
} 
Summing the residues, one obtains:

$$
\begin{aligned}
I= & \frac{1}{N !} \sum_{\vec{\gamma} \in\left(\mathbb{Z}_{N_{f}}\right)^{N}} \sum_{\vec{\mu}, \vec{\nu} \in \mathbb{Z}_{\geq 0}^{N}}(-1)^{-k \sum_{j}\left(\mu_{j}-\nu_{j}\right)} w^{\sum_{j}\left(\mu_{j}-\nu_{j}\right)} \prod_{i=1}^{N}\left(\zeta_{\gamma_{i}}^{-1} x^{\mu_{i}+\nu_{i}}\right)^{-k\left(\mu_{i}-\nu_{i}\right)+\mathfrak{n}} \\
& \times \prod_{i \neq j}^{N} x^{-\frac{1}{2}\left|\left(\mu_{i}-\nu_{i}\right)-\left(\mu_{j}-\nu_{j}\right)\right|}\left(1-\frac{\zeta_{\gamma_{j}}^{-1} x^{\mu_{j}+\nu_{j}}}{\zeta_{\gamma_{i}}^{-1} x^{\mu_{i}+\nu_{i}}} x^{\left|\left(\mu_{i}-\nu_{i}\right)-\left(\mu_{j}-\nu_{j}\right)\right|}\right) \prod_{i=1}^{N} \frac{\left(x^{\mu_{i}+\nu_{i}+1}\right)^{-\left(\mu_{i}-\nu_{i}\right) / 2}}{\left(x^{-2} ; x^{-2}\right)_{\mu_{i}}\left(x^{2} ; x^{2}\right)_{\nu_{i}}} \\
& \times \prod_{i=1}^{N} \prod_{\alpha\left(\neq \gamma_{i}\right)}^{N_{f}}\left(\zeta_{\alpha} \zeta_{\gamma_{i}}^{-1} x^{\mu_{i}+\nu_{i}+1}\right)^{-\left(\mu_{i}-\nu_{i}\right) / 2} \frac{\left(\zeta_{\alpha} \zeta_{\gamma_{i}}^{-1} x^{2 \nu_{i}+2} ; x^{2}\right)_{\infty}}{\left(\zeta_{\alpha}^{-1} \zeta_{\gamma_{i}} x^{-2 \mu_{i}} ; x^{2}\right)_{\infty}} \\
& \times \prod_{i=1}^{N} \prod_{\beta=1}^{N_{a}}\left(\tilde{\zeta}_{\beta}^{-1} \zeta_{\gamma_{i}} x^{-\mu_{i}-\nu_{i}+1}\right)^{\left(\mu_{i}-\nu_{i}\right) / 2} \frac{\left(\tilde{\zeta}_{\beta}^{-1} \zeta_{\gamma_{i}} x^{-2 \nu_{i}+2} ; x^{2}\right)_{\infty}}{\left(\tilde{\zeta}_{\beta} \zeta_{\gamma_{i}}^{-1} x^{2 \mu_{i}} ; x^{2}\right)_{\infty}}
\end{aligned}
$$

where we decomposed the summation over $\mu_{i}=r_{i}+\frac{\mathfrak{m}_{i}+\left|\mathfrak{m}_{i}\right|}{2}$ and $\nu_{i}=\mu_{i}-\mathfrak{m}_{i}$. The $q$-Pochhammer symbol is $(a ; q)_{n}=\prod_{k=0}^{n-1}\left(1-q^{k} a\right)$.

At this point one can factorize the summation into a factor independent of $\vec{\mu}$ and $\vec{\nu}$, a summation over $\vec{\mu}$ and a summation over $\vec{\nu}$. One observes that each of the two summations over $\vec{\mu}$ and $\vec{\nu}$ vanishes if we choose $\gamma_{i}=\gamma_{j}$ for some $i, j$, and on the other hand it is symmetric under permutations of the $\gamma_{i}$ 's. Therefore we can restrict the sum over unordered combinations $\vec{\gamma} \in C\left(N, N_{f}\right)$ of $N$ out of the $N_{f}$ flavors, and cancel the $N$ ! in the denominator. Finally, rewriting the $q$-Pochhammer symbols in terms of sinh and using the identity (2.73) one obtains

$$
I=\sum_{\vec{\gamma} \in C\left(N, N_{f}\right)} Z_{\mathrm{cl}}^{(\vec{\gamma})} Z_{1-\mathrm{loop}}^{(\vec{\gamma})} Z_{\mathrm{v}}^{(\vec{\gamma})} Z_{\mathrm{av}}^{(\vec{\gamma})}
$$

The classical and one-loop contributions are

$$
\begin{aligned}
Z_{\mathrm{cl}}^{(\vec{\gamma})} & =\prod_{j \in \vec{\gamma}} \zeta_{j}^{-\mathfrak{n}} \\
Z_{1-\text { loop }}^{\prime(\vec{\gamma})} & =\prod_{j \in \vec{\gamma}} \prod_{\alpha(\neq j)}^{N_{f}} \frac{\left(\zeta_{j}^{-1} \zeta_{\alpha} x^{2} ; x^{2}\right)_{\infty}}{\left(\zeta_{j} \zeta_{\alpha}^{-1} ; x^{2}\right)_{\infty}} \prod_{\beta=1}^{N_{a}} \frac{\left(\zeta_{j} \tilde{\zeta}_{\beta}^{-1} x^{2} ; x^{2}\right)_{\infty}}{\left(\zeta_{j}^{-1} \tilde{\zeta}_{\beta} ; x^{2}\right)_{\infty}} \cdot \prod_{\substack{i, j \in \vec{\gamma} \\
i \neq j}} 2 \sinh \left(\frac{i_{\mathfrak{z} i}-i \mathfrak{z}_{j} j}{2}\right) .
\end{aligned}
$$

The vortex and antivortex contribution can be written as

$$
\begin{aligned}
& Z_{\mathrm{v}}^{(\vec{\gamma})}=Z_{\operatorname{vortex}}^{(\vec{\gamma})}\left(e^{-\xi k},\left.w_{\mathrm{v}}^{-1} e^{\left(-i \mathfrak{z}_{j} k-\xi \mathfrak{n}\right)}\right|_{j \in \vec{\gamma}},-2 i \xi, i \mathfrak{z}_{\alpha}, i \tilde{\mathfrak{z}}_{\beta}\right) \\
& Z_{\mathrm{av}}^{(\vec{\gamma})}=Z_{\operatorname{vortex}}^{(\vec{\gamma})}\left(e^{\xi k},\left.w_{\mathrm{av}} e^{\left(i_{\mathfrak{z}} k-\xi \mathfrak{n}\right)}\right|_{i \in \vec{\gamma}}, 2 i \xi,-i \mathfrak{z}_{\alpha},-i \tilde{\mathfrak{z}}_{\beta}\right),
\end{aligned}
$$

and the vortex-string partition function turns out to be exactly the same (2.77) as for the 
computation on $S_{b}^{3}$, namely:

$$
\begin{aligned}
Z_{\text {vortex }}^{(\vec{\gamma})}\left(Q_{j}, L_{j}, \varepsilon, a_{\alpha}, b_{\beta}\right)=\sum_{\vec{\mu} \in \mathbb{Z}_{\geq 0}^{N}} \prod_{j \in \vec{\gamma}} Q_{j}^{\mu_{j}^{2}} L_{j}^{\mu_{j}}(-1)^{\left(N_{f}-N_{a}\right) \mu_{j}} \\
\quad \times \prod_{\lambda=0}^{\mu_{j}-1} \frac{\prod_{\beta=1}^{N_{a}} 2 i \sinh \frac{a_{j}-b_{\beta}+i \varepsilon \lambda}{2}}{\prod_{l \in \vec{\gamma}} 2 i \sinh \frac{a_{j}-a_{l}+i \varepsilon\left(\lambda-\mu_{l}\right)}{2} \prod_{\alpha \notin \vec{\gamma}}^{N_{f}} 2 i \sinh \frac{a_{\alpha}-a_{j}+i \varepsilon\left(\lambda-\mu_{j}\right)}{2}} .
\end{aligned}
$$

The fugacity $w$ for the topological charge is rotated by a phase: $w_{\mathrm{v}}=$ $(-i)^{N_{f}-N_{a}}(-1)^{k+N-1} w, w_{\mathrm{av}}=i^{N_{f}-N_{a}}(-1)^{k+N-1} w$. The parameters that determine $Z_{\mathrm{v}}$ and $Z_{\mathrm{av}}$ in terms of $Z_{\mathrm{vortex}}$ are exactly as prescribed by our general discussion in section 3.4.4.

\section{Discussion}

In this paper we have extended the Higgs branch localization framework of [4] to threedimensional $\mathcal{N}=2$ R-symmetry theories on $S_{b}^{3}$ and $S^{2} \times S^{1}$. We expect the method to work on much more general $3 \mathrm{~d}$ backgrounds. We also expect a possible further extension to fourdimensional $\mathcal{N}=1$ theories on manifolds like $S^{3} \times S^{1}$ or $S^{2} \times T^{2}$ (and fibrations thereof) which naturally support vortex-membranes, i.e. vortices with $2 \mathrm{~d}$ worldvolume. Even more generally, the method should work for theories with 8 supercharges, for instance in 4 and 5 dimensions. We leave these investigations to future work.

Higgs branch localization expresses the partition function in terms of the (3d version of the) vortex partition function (VPF), which could also be computed in the $\Omega$ background $[25,26,36]$. In fact, the partition function on different geometries - like $S_{b}^{3}$ and $S^{2} \times S^{1}$-is controlled by the very same VPF, with different identifications of the parameters. This has been extensively elaborated upon in [28].

It might be worth studying more in detail aspects of the 3d VPF. For instance, 3d mirror symmetry maps particles to vortices [51] and it would be interesting to understand its action on the VPF. Through the mirror map [58] between star-shaped quivers and the $3 \mathrm{~d}$ reduction of class- $S$ theories $[59,60]$, this might shed more light on the latter.

Finally, the VPF encodes (equivariant) geometrical information about the Higgs branch of the theory. It might be interesting to investigate how the VPF captures the quantum moduli space [61-64] of Chern-Simons-matter quiver theories arising from M2-branes at Calabi-Yau fourfold singularities.

\section{Acknowledgments}

The authors would like to thank Takuya Okuda, Sara Pasquetti and Leonardo Rastelli for useful discussions and correspondence. F.B. would also like to thank Dario Martelli and James Sparks for discussion on related material. F.B.'s work is supported in part by DOE grant DE-FG02-92ER-40697. W.P. is supported in part by NSF Grant PHY-0969919. 


\section{A Spinor conventions}

We use essentially the same conventions as in $[4,11,12]$. In vielbein space we take the gamma matrices $\gamma^{a}=\left(\begin{array}{ll}0 & 1 \\ 1 & 0\end{array}\right),\left(\begin{array}{cc}0 & -i \\ i & 0\end{array}\right),\left(\begin{array}{cc}1 & 0 \\ 0 & -1\end{array}\right)$ which do not have definite symmetry: $\left[\gamma^{1}, \gamma^{2}, \gamma^{3}\right]^{\top}=\left[\gamma^{1},-\gamma^{2}, \gamma^{3}\right]$. We take the charge conjugation matrix $C$, defined by $C \gamma^{\mu} C^{-1}=-\gamma^{\mu \top}$, as $C=-i \varepsilon_{\alpha \beta}=\gamma_{2}\left(\right.$ where $\left.\varepsilon_{12}=\varepsilon^{12}=1\right)$ so that

$$
C \gamma^{\mu} C=-\gamma^{\mu \top}, \quad C^{2}=\mathbb{1}
$$

Indeed $C=C^{-1}=C^{\dagger}=-C^{\top}=-C^{*}$. Since Dirac spinors are in the $\mathbf{2}$ of $\mathrm{SU}(2)$, there are two products we can consider: $\eta^{\top} C \epsilon \equiv-i \eta^{\alpha} \varepsilon_{\alpha \beta} \epsilon^{\beta}$ and $\eta^{\dagger} \epsilon \equiv \eta_{\alpha}^{*} \epsilon^{\alpha}$. When we use the first product, we omit ${ }^{\top} C$ (that is we write $\eta \epsilon \equiv \eta^{\top} C \epsilon$ ). The two products are related by charge conjugation: $\epsilon^{c} \equiv C \epsilon^{*}$ and $\epsilon^{c \dagger}=\epsilon^{\top} C$, so that $\eta^{\top} C \epsilon=\eta^{c \dagger} \epsilon$. Notice that $\left(\epsilon^{c}\right)^{c}=-\epsilon$ and there are no Majorana spinors.

Barred spinors will simply be independent spinors. Products are constructed as spelled out before: $\bar{\epsilon} \lambda \equiv \bar{\epsilon}^{\alpha} C_{\alpha \beta} \lambda^{\beta}, \bar{\epsilon} \gamma^{\mu} \lambda \equiv \bar{\epsilon}^{\alpha}\left(C \gamma^{\mu}\right)_{\alpha \beta} \lambda^{\beta}$, etc... The charge conjugation matrix $C$ is antisymmetric, while $C \gamma^{a}$ are symmetric and so $C \gamma^{\mu}$. Since $\gamma^{\mu \nu}$ equals a single gamma matrix or zero, also $C \gamma^{\mu \nu}$ are symmetric. For anticommuting fermions we get:

$$
\bar{\epsilon} \lambda=\lambda \bar{\epsilon}, \quad \bar{\epsilon} \gamma^{\mu} \lambda=-\lambda \gamma^{\mu} \bar{\epsilon}, \quad \bar{\epsilon} \gamma^{\mu \nu} \lambda=-\lambda \gamma^{\mu \nu} \bar{\epsilon}
$$

Some useful relations among gamma matrices are:

$$
\begin{array}{rlrl}
{\left[\gamma_{\mu}, \gamma_{\nu}\right]} & =2 g_{\mu \nu}, & \gamma_{\mu} \gamma_{\nu}=g_{\mu \nu}+\gamma_{\mu \nu}, \quad \gamma^{\mu \nu} & =i \varepsilon^{\mu \nu \rho} \gamma_{\rho}, \quad \gamma^{\mu \nu} \varepsilon_{\mu \nu \rho}=2 i \gamma_{\rho} \\
\gamma_{\mu} \gamma^{\nu \rho} & =i \varepsilon^{\nu \rho \sigma} g_{\mu \sigma}+\left(\delta_{\mu}^{\nu} \delta_{\alpha}^{\rho}-\delta_{\alpha}^{\nu} \delta_{\mu}^{\rho}\right) \gamma^{\alpha}, & \gamma_{\mu \nu} \gamma^{\nu}=-\gamma^{\nu} \gamma_{\mu \nu}=2 \gamma_{\mu} \\
\gamma_{\mu} \gamma^{\nu \rho} \gamma^{\mu} & =-\gamma^{\nu \rho}, \quad \gamma_{\mu} \gamma^{\nu} \gamma^{\mu}=-\gamma^{\nu}, & \gamma^{\mu} \gamma_{\mu}=3, \\
\gamma^{\mu \nu} \gamma_{\rho} \gamma_{\nu} & =-2 \delta_{\rho}^{\mu}, \quad \gamma^{\mu \nu} \gamma_{\rho} \gamma_{\mu \nu}=2 \gamma_{\rho} .
\end{array}
$$

The antisymmetric tensor with flat indices is $\varepsilon^{\hat{1} \hat{2} \hat{3}}=\varepsilon_{\hat{1} \hat{2} \hat{3}}=1$, and the covariant forms with curved indices are $\varepsilon_{\mu \nu \rho}=\sqrt{g} \varepsilon_{\hat{\mu} \hat{\nu} \hat{\rho}}$ and $\varepsilon^{\mu \nu \rho}=\frac{1}{\sqrt{g}} \varepsilon^{\hat{\mu} \hat{\nu} \hat{\rho}}$.

The Fierz identity for anticommuting $3 \mathrm{~d}$ Dirac fermions is

$$
\left(\bar{\lambda}_{1} \lambda_{2}\right) \lambda_{3}=-\frac{1}{2}\left(\bar{\lambda}_{1} \lambda_{3}\right) \lambda_{2}-\frac{1}{2}\left(\bar{\lambda}_{1} \gamma^{\rho} \lambda_{3}\right) \gamma_{\rho} \lambda_{2} .
$$

Since $\gamma^{\alpha}$ and $\gamma^{\mu \nu}$ are dual, one finds

$$
\left(\gamma_{\mu \rho}\right)_{* *}\left(\gamma^{\rho}\right)_{* *}=\left(\gamma^{\rho}\right)_{* *}\left(\gamma_{\rho \mu}\right)_{* *}, \quad-2\left(\gamma_{\mu}\right)_{* *}\left(\gamma^{\mu}\right)_{* *}=\left(\gamma_{\nu \rho}\right)_{* *}\left(\gamma^{\nu \rho}\right)_{* *}
$$

where indices are not contracted. It might also be useful:

$$
-\frac{i}{4} \bar{\epsilon} \gamma^{\rho} \gamma^{\mu \nu} \epsilon \gamma_{\rho} \gamma_{\nu} \mathcal{O}_{\mu} \lambda=-\frac{i}{2} \bar{\epsilon} \epsilon \gamma^{\mu} \mathcal{O}_{\mu} \lambda+\frac{i}{2} \bar{\epsilon} \gamma^{\mu} \epsilon \mathcal{O}_{\mu} \lambda+\frac{i}{4} \bar{\epsilon} \gamma^{\alpha \rho} \epsilon \gamma_{\rho} \mathcal{O}_{\alpha} \lambda,
$$

where $\mathcal{O}_{\mu}$ is any operator, acting on any field. 


\section{B Supersymmetric theories on three-manifolds}

Following [11, 12], we write the superconformal transformation rules on the gauge and matter multiplets on a three-dimensional manifold. The manifold is restricted by the requirement that it admits solutions to the usual Killing spinor equations, and that the superalgebra closes. After presenting the supersymmetry variations, in section B.2 we present the anticommuting supercharges by replacing the anticommuting Killing spinors in $\delta_{\epsilon}$ and $\delta_{\bar{\epsilon}}$ with their commuting counterparts. Lagrangians invariant under the supersymmetry transformations were studied in $[11,12]$. Most of them are exact and therefore will not contribute in a localization computation. Notable exceptions are the Chern-Simons and Fayet-Iliopoulos actions. A more systematic analysis of SUSY on three-manifolds has been done in $[34,35]$.

\section{B.1 The superconformal algebra}

We define the field strength as $F_{\mu \nu}=\partial_{\mu} A_{\nu}-\partial_{\nu} A_{\mu}-i\left[A_{\mu}, A_{\nu}\right]$, and the gauge and metric covariant derivative as $D_{\mu}=\nabla_{\mu}-i A_{\mu}$, where $\nabla_{\mu}$ is the metric-covariant derivative. It follows, for instance, that for an adjoint scalar $\sigma:\left[D_{\mu}, D_{\nu}\right] \sigma=-i\left[F_{\mu \nu}, \sigma\right]$. We will also turn on a background gauge field $V_{\mu}$ for $\mathrm{U}(1)_{R}$, therefore

$$
D_{\mu}=\nabla_{\mu}-i A_{\mu}-i V_{\mu} .
$$

The superconformal transformations of the vector multiplet are

$$
\begin{array}{rlr}
\delta A_{\mu} & =-\frac{i}{2}\left(\bar{\epsilon} \gamma_{\mu} \lambda-\bar{\lambda} \gamma_{\mu} \epsilon\right) & \delta \sigma=\frac{1}{2}(\bar{\epsilon} \lambda-\bar{\lambda} \epsilon) \\
\delta \lambda & =\frac{1}{2} \gamma^{\mu \nu} \epsilon F_{\mu \nu}-D \epsilon+i \gamma^{\mu} \epsilon D_{\mu} \sigma+\frac{2 i}{3} \sigma \gamma^{\mu} D_{\mu} \epsilon & \\
\delta \bar{\lambda} & =\frac{1}{2} \gamma^{\mu \nu} \bar{\epsilon} F_{\mu \nu}+D \bar{\epsilon}-i \gamma^{\mu} \bar{\epsilon} D_{\mu} \sigma-\frac{2 i}{3} \sigma \gamma^{\mu} D_{\mu} \bar{\epsilon} & \\
\delta D & =-\frac{i}{2} \bar{\epsilon} \gamma^{\mu} D_{\mu} \lambda-\frac{i}{2} D_{\mu} \bar{\lambda} \gamma^{\mu} \epsilon+\frac{i}{2}[\bar{\epsilon} \lambda, \sigma]+\frac{i}{2}[\bar{\lambda} \epsilon, \sigma]-\frac{i}{6}\left(D_{\mu} \bar{\epsilon} \gamma^{\mu} \lambda+\bar{\lambda} \gamma^{\mu} D_{\mu} \epsilon\right),
\end{array}
$$

and those of the chiral multiplet are

$$
\begin{array}{ll}
\delta \phi=\bar{\epsilon} \psi & \delta \psi=i \gamma^{\mu} \epsilon D_{\mu} \phi+i \epsilon \sigma \phi+\frac{2 i q}{3} \gamma^{\mu} D_{\mu} \epsilon \phi+\bar{\epsilon} F \\
\delta \bar{\phi}=\bar{\psi} \epsilon & \delta \bar{\psi}=i \gamma^{\mu} \bar{\epsilon} D_{\mu} \bar{\phi}+i \bar{\epsilon} \bar{\phi} \sigma+\frac{2 i q}{3} \gamma^{\mu} D_{\mu} \bar{\epsilon} \bar{\phi}+\epsilon \bar{F} \\
\delta F=\epsilon\left(i \gamma^{\mu} D_{\mu} \psi-i \sigma \psi-i \lambda \phi\right)+\frac{i}{3}(2 q-1) D_{\mu} \epsilon \gamma^{\mu} \psi \\
\delta \bar{F}=\bar{\epsilon}\left(i \gamma^{\mu} D_{\mu} \bar{\psi}-i \bar{\psi} \sigma+i \bar{\phi} \bar{\lambda}\right)+\frac{i}{3}(2 q-1) D_{\mu} \bar{\epsilon} \gamma^{\mu} \bar{\psi}
\end{array}
$$

Here $\epsilon$ and $\bar{\epsilon}$ are independent spinors satisfying the Killing spinor equations

$$
D_{\mu} \epsilon=\gamma_{\mu} \hat{\epsilon}, \quad D_{\mu} \bar{\epsilon}=\gamma_{\mu} \hat{\bar{\epsilon}},
$$

in terms of some other spinors $\hat{\epsilon}, \hat{\bar{\epsilon}}$. Closure of the algebra requires the additional constraints:

$$
\gamma^{\mu} \gamma^{\nu} D_{\mu} D_{\nu} \epsilon=-\frac{3}{8}\left(R-2 i V_{\mu \nu} \gamma^{\mu \nu}\right) \epsilon, \quad \gamma^{\mu} \gamma^{\nu} D_{\mu} D_{\nu} \bar{\epsilon}=-\frac{3}{8}\left(R+2 i V_{\mu \nu} \gamma^{\mu \nu}\right) \bar{\epsilon}
$$


with the same functions $R$ and $V_{\mu \nu}[11,12]$. Consistency implies that $R$ is the scalar curvature of the three-manifold and $V_{\mu \nu}=\partial_{\mu} V_{\nu}-\partial_{\nu} V_{\mu}$ is the background gauge field strength. Then the algebra reads

$$
\left[\delta_{\epsilon}, \delta_{\bar{\epsilon}}\right]=\mathcal{L}_{\xi}^{A}+i \Lambda+\rho \Delta+i \alpha R, \quad\left[\delta_{\epsilon}, \delta_{\epsilon}\right]=0, \quad\left[\delta_{\bar{\epsilon}}, \delta_{\bar{\epsilon}}\right]=0
$$

where $\mathcal{L}_{\xi}^{A}$ is the gauge-covariant Lie derivative (independent of the metric, see below) along the vector field $\xi, i \Lambda$ denotes a gauge transformation with parameter $i \Lambda, R$ is the R-symmetry charge, ${ }^{17}$ and $\Delta$ the scaling weight. ${ }^{18}$ The parameters themselves are given by

$$
\begin{aligned}
\xi^{\mu} & =i \bar{\epsilon} \gamma^{\mu} \epsilon & \rho & =\frac{i}{3}\left(D_{\mu} \bar{\epsilon} \gamma^{\mu} \epsilon+\bar{\epsilon} \gamma^{\mu} D_{\mu} \epsilon\right)=\frac{1}{3} D_{\mu} \xi^{\mu} \\
\Lambda & =\bar{\epsilon} \epsilon \sigma & \alpha & =-\frac{1}{3}\left(D_{\mu} \bar{\epsilon} \gamma^{\mu} \epsilon-\bar{\epsilon} \gamma^{\mu} D_{\mu} \epsilon\right)-\xi^{\mu} V_{\mu}
\end{aligned}
$$

The Lie derivative $\mathcal{L}_{X}$ with respect to a vector field $X$ is a derivation independent of the metric. On forms it is easily defined as $\mathcal{L}_{X}=\left\{d, \iota_{X}\right\}$ in terms of the contraction $\iota_{X}$; using the normalization $\alpha=\frac{1}{n !} \alpha_{\mu_{1} \cdots \mu_{n}} d x^{\mu_{1} \cdots \mu_{n}}$, in components we have

$$
\left[\mathcal{L}_{X} \alpha\right]_{\mu_{1} \cdots \mu_{n}}=X^{\mu} \partial_{\mu} \alpha_{\mu_{1} \cdots \mu_{n}}+n\left(\partial_{\left[\mu_{1}\right.} X^{\mu}\right) \alpha_{\left.\mu \mid \mu_{2} \cdots \mu_{n}\right]} .
$$

The Lie derivative of spinors [65] (see [66] for explanations) is

$$
\mathcal{L}_{X} \psi=X^{\mu} \nabla_{\mu} \psi+\frac{1}{4} \nabla_{\mu} X_{\nu} \gamma^{\mu \nu} \psi
$$

where the covariant derivative is $\nabla_{\mu}=\partial_{\mu}+\frac{1}{4} \omega_{\mu}^{a b} \gamma_{a b}$. Although this definition seems to depend on the metric (through the spin connection and the vielbein), the dependence in fact cancels out. Finally, we can define a "gauge-covariant" Lie derivative that acts on sections of some (gauge) vector bundle. On tensors it is simply obtained by substituting the flat derivative $\partial_{\mu}$ with the covariant derivative, $\partial_{\mu} \rightarrow \partial_{\mu}^{A}=\partial_{\mu}-i A_{\mu}$, while on spinors it is obtained by substituting $\nabla_{\mu} \rightarrow \nabla_{\mu}^{A}$ in the first term. The gauge-covariant Lie derivative of the connection (which does not transform as a section of the adjoint bundle) is defined as

$$
\mathcal{L}_{X}^{A} A=\mathcal{L}_{X} A-d^{A}\left(\iota_{X} A\right), \quad\left(\mathcal{L}_{X}^{A} A\right)_{\mu}=X^{\rho} F_{\rho \mu}=X^{\rho}\left(2 \partial_{[\rho} A_{\mu]}-i\left[A_{\rho}, A_{\mu}\right]\right) .
$$

\footnotetext{
${ }^{17}$ The R-charges are:

$R\left(A_{\mu}, \sigma, \lambda, \bar{\lambda}, D\right)=(0,0,-1,1,0), R(\phi, \bar{\phi}, \psi, \bar{\psi}, F, \bar{F})=(q,-q, q-1,1-q, q-2,2-q), \quad R(\epsilon, \bar{\epsilon})=(-1,+1)$
}

${ }^{18}$ The dilation weights are:

$$
\begin{aligned}
\Delta\left(A_{\mu}, \sigma, \lambda, \bar{\lambda}, D\right) & =\left(1,1, \frac{3}{2}, \frac{3}{2}, 2\right), \Delta(\phi, \bar{\phi}, \psi, \bar{\psi}, F, \bar{F})=\left(q, q, q+\frac{1}{2}, q+\frac{1}{2}, q+1, q+1\right), \\
\Delta(\epsilon, \bar{\epsilon}) & =\left(\frac{1}{2}, \frac{1}{2}\right) .
\end{aligned}
$$

Note that whereas the 1 -form $A$ has weight zero, its components have weight 1 . The commutator on $A_{\mu}$ gives the $\mu$-component of the Lie derivative on the 1-form $A$, without further action of the dilation group. 


\section{B.2 Commuting Killing spinors}

For given anticommuting spinors $\epsilon, \bar{\epsilon}$, let us construct the corresponding supercharges $Q, \tilde{Q}$ in terms of commuting spinors $\epsilon$ and $\tilde{\epsilon}=-C \bar{\epsilon}^{*}$ (so that $\bar{\epsilon}=\tilde{\epsilon}^{c}$ ). They are constructed as follows:

$$
\delta=\delta_{\epsilon}+\delta_{\bar{\epsilon}}=\epsilon^{\alpha} Q_{\alpha}+\bar{\epsilon}^{\alpha} \tilde{Q}_{\alpha}, \quad Q=\epsilon^{\alpha} Q_{\alpha}, \quad \tilde{Q}=\tilde{\epsilon}^{c \alpha} \tilde{Q}_{\alpha}=-\left(\tilde{\epsilon}^{\dagger} C\right)^{\alpha} \tilde{Q}_{\alpha} .
$$

We also need the charge conjugate $\bar{\lambda}=C\left(\lambda^{\dagger}\right)^{\top}$. On the vector multiplet we get:

$$
\begin{array}{rlrlrl}
Q A_{\mu} & =\frac{i}{2} \lambda^{\dagger} \gamma_{\mu} \epsilon & Q \lambda & =\frac{1}{2} \gamma^{\mu \nu} \epsilon F_{\mu \nu}-D \epsilon+i \gamma^{\mu} \epsilon D_{\mu} \sigma+\frac{2 i}{3} \sigma \gamma^{\mu} D_{\mu} \epsilon \\
\tilde{Q} A_{\mu} & =\frac{i}{2} \tilde{\epsilon}^{\dagger} \gamma_{\mu} \lambda & \tilde{Q} \lambda^{\dagger} & =-\frac{1}{2} \tilde{\epsilon}^{\dagger} \gamma^{\mu \nu} F_{\mu \nu}+\tilde{\epsilon}^{\dagger} D+i \tilde{\epsilon}^{\dagger} \gamma^{\mu} D_{\mu} \sigma+\frac{2 i}{3} D_{\mu} \tilde{\epsilon}^{\dagger} \gamma^{\mu} \sigma \\
Q D & =-\frac{i}{2} D_{\mu} \lambda^{\dagger} \gamma^{\mu} \epsilon+\frac{i}{2}\left[\lambda^{\dagger} \epsilon, \sigma\right]-\frac{i}{6} \lambda^{\dagger} \gamma^{\mu} D_{\mu} \epsilon & \tilde{Q} \lambda=0 & Q \sigma=-\frac{1}{2} \lambda^{\dagger} \epsilon \\
\tilde{Q} D & =\frac{i}{2} \tilde{\epsilon}^{\dagger} \gamma^{\mu} D_{\mu} \lambda+\frac{i}{2}\left[\sigma, \tilde{\epsilon}^{\dagger} \lambda\right]+\frac{i}{6} D_{\mu} \tilde{\epsilon}^{\dagger} \gamma^{\mu} \lambda & Q \lambda^{\dagger}=0 & \tilde{Q} \sigma=-\frac{1}{2} \tilde{\epsilon}^{\dagger} \lambda .
\end{array}
$$

On the chiral multiplet we get:

$$
\begin{array}{rlrl}
Q \phi & =0 & \tilde{Q} \phi & =-\tilde{\epsilon}^{\dagger} \psi \\
Q \phi^{\dagger} & =\psi^{\dagger} \epsilon & \tilde{Q} \phi^{\dagger} & =0 \\
Q \psi & =\left(i \gamma^{\mu} D_{\mu} \phi+i \sigma \phi\right) \epsilon+\frac{2 i q}{3} \phi \gamma^{\mu} D_{\mu} \epsilon & \tilde{Q} \psi & =C \tilde{\epsilon}^{*} F \\
\tilde{Q} \psi^{\dagger} & =\tilde{\epsilon}^{\dagger}\left(-i \gamma^{\mu} D_{\mu} \phi^{\dagger}+i \phi^{\dagger} \sigma\right)-\frac{2 i q}{3} D_{\mu} \tilde{\epsilon}^{\dagger} \gamma^{\mu} \phi^{\dagger} & Q \psi^{\dagger} & =-\epsilon^{\top} C F^{\dagger} \\
Q F & =\epsilon^{\top} C\left(i \gamma^{\mu} D_{\mu} \psi-i \sigma \psi-i \lambda \phi\right)+\frac{i(2 q-1)}{3} D_{\mu} \epsilon^{\top} C \gamma^{\mu} \psi & \tilde{Q} F & =0 \\
\tilde{Q} F^{\dagger} & =\left(-i D_{\mu} \psi^{\dagger} \gamma^{\mu}-i \psi^{\dagger} \sigma+i \phi^{\dagger} \lambda^{\dagger}\right) C \epsilon^{*}-\frac{i(2 q-1)}{3} \psi^{\dagger} \gamma^{\mu} C D_{\mu} \tilde{\epsilon}^{*} & Q F^{\dagger} & =0 .
\end{array}
$$

Finally we define $\mathcal{Q} \equiv Q+\tilde{Q}$.

\section{B.3 Supersymmetric actions}

Let us write down the $\mathcal{Q}$-closed but not $\mathcal{Q}$-exact actions we consider in the paper: they are the Chern-Simons (CS) action and the Fayet-Iliopoulos (FI) action. Since they are nontrivial in $\mathcal{Q}$-cohomology, their evaluation on the BPS configurations is non-trivial. The CS action is

$$
S_{\mathrm{CS}}=-\frac{i}{4 \pi} \int \operatorname{Tr}_{\mathrm{CS}}\left[A \wedge F-\frac{2 i}{3} A \wedge A \wedge A+(2 D \sigma-\bar{\lambda} \lambda) d \mathrm{vol}\right]
$$

both on $S_{b}^{3}$ and $S^{2} \times S^{1}$. The symbol $\operatorname{Tr}_{\mathrm{CS}}$ (as in [9]) means a trace where each Abelian and simple factor in the gauge group is weighed by its own (quantized) CS level $k$. For instance, for $\mathrm{SU}(N)$ this would just be $\operatorname{Tr}_{\mathrm{CS}}=k \operatorname{Tr}$.

The FI action on $S_{b}^{3}$ is

$$
S_{\mathrm{FI}}=\frac{i}{2 \pi \sqrt{\ell \tilde{\ell}}} \int \operatorname{Tr}_{\mathrm{FI}}\left(D-\frac{\sigma}{f}\right) d \operatorname{vol}\left(S_{b}^{3}\right),
$$

where again $\operatorname{Tr}_{\mathrm{FI}}$ is a trace where each Abelian factor is weighed by its own FI term $\xi$. For $\mathrm{U}(N)$, this would just be $\operatorname{Tr}_{\mathrm{FI}}=\xi \operatorname{Tr}$. 


\section{One-loop determinants from an index theorem}

The one-loop determinants of quadratic fluctuations around a non-trivial background, in particular around our general vortex backgrounds, are most easily evaluated with the help of an equivariant index theorem for transversally elliptic operators [45]. Such a technique was used on $S^{4}[3,47]$ and $S^{2}$ [4], while the computations on $S_{b}^{3}$ and $S^{2} \times S^{1}$ have been done in [46]. We will summarize the latter computation here, adapted to our conventions, referring to $[3,4,46,47]$ for details.

After the cancelations between bosons and fermions, the one-loop determinant equals the ratio $\operatorname{det}_{\text {coker }} D_{o e} \mathcal{Q}^{2} / \operatorname{det}_{\text {ker }} D_{o e} \mathcal{Q}^{2}$, where $D_{o e}$ is the projection, from a subset $\left\{\varphi_{e}\right\}$ to a subset $\left\{\varphi_{o}\right\}$ of fields, of the expansion of $\mathcal{Q}$ at linear order around the background. The ratio of weights of the group action of $\mathcal{Q}^{2}$ on respective spaces can be computed by first evaluating the index

$$
\text { ind } D_{o e}(\epsilon)=\operatorname{tr}_{\text {ker } D_{o e}} e^{\mathcal{Q}^{2}(\epsilon)}-\operatorname{tr}_{\text {coker } D_{o e}} e^{\mathcal{Q}^{2}(\epsilon)},
$$

where $\epsilon$ summarizes the equivariant parameters, and then extracting the determinant with the map

$$
\sum_{\alpha} c_{\alpha} e^{w_{\alpha}(\epsilon)} \rightarrow \prod_{\alpha} w_{\alpha}(\epsilon)^{c_{\alpha}}
$$

As explained in the main text, ind $D_{o e}(\epsilon)$ is computed with the help of the index theorem, and it only gets contributions from the fixed points on the worldvolume of the action of $\mathcal{Q}^{2}$. However the theorem can be applied if the action is compact, which is not the case on $S_{b}^{3}$ and $S^{2} \times S^{1}$ in general. Then [46] propose to reduce along an $S^{1}$ fiber, and be left with the computation on $S^{2}$, as in [4]. It turns out that for the chiral multiplet the operator $D_{o e}$ is the Dolbeault operator $D_{\bar{z}}$ with inverted grading acting on $\Omega^{(0,0)}$, whose index is $-\frac{1}{1-z}$, while for the vector multiplet it is the real operator $d^{*} \oplus d$ acting on $\Omega^{1}$, whose index is $\frac{1}{2}$.

The sphere $\boldsymbol{S}_{\boldsymbol{b}}^{\mathbf{3}}$. We write the metric in Hopf coordinates as in (2.12), in terms of $\phi_{H}=\varphi-\chi$ and $\psi_{H}=\varphi+\chi$. The square of the supercharge is

$$
\begin{aligned}
\mathcal{Q}^{2} & =\mathcal{L}_{\xi}^{A}-\sigma-\frac{i}{2}\left(\frac{1}{\ell}+\frac{1}{\tilde{\ell}}\right) R=\frac{b}{r} \mathcal{L}_{\partial_{\varphi}}^{A}+\frac{b^{-1}}{r} \mathcal{L}_{\partial_{\chi}}^{A}-\frac{r \sigma}{r}-\frac{i}{2 r}\left(b+b^{-1}\right) R \\
& =\frac{b+b^{-1}}{r} \mathcal{L}_{\psi_{H}}^{A}+\frac{b-b^{-1}}{r} \mathcal{L}_{\phi_{H}}^{A}-\frac{r \sigma}{r}-\frac{i}{2 r}\left(b+b^{-1}\right) R
\end{aligned}
$$

where we used $r=\sqrt{\ell \tilde{\ell}}$ and $b=\sqrt{\tilde{\ell} / \ell}$.

At the northern circle, $\theta=0$, the Hopf fiber is parametrized by $\varphi($ see $(2.1))$ and $\mathcal{Q}^{2}$ acts freely on it with equivariant parameter $b$; the KK modes thus contribute $\sum_{n \in \mathbb{Z}} e^{i b n}$ to the index. On the $S^{2}$, parametrized by $\theta$ and $\phi_{H}$, resulting from the reduction along the Hopf fiber, $\mathcal{Q}^{2}$ has a fixed point at $\theta=0$. There the SUSY variation of a chiral multiplet (see (2.30)) is schematically $D_{\theta}+\frac{i}{\theta} D_{\phi_{H}} \sim D_{\bar{z}}$ if we identify $z=\theta e^{i \phi_{H}}$. In fact the one-loop determinant of the chiral multiplet is the index of the Dolbeault operator with inverted grading (as noticed in $[3,4,47]$ ), which is $-\frac{1}{1-z}$. Now we expand in $t=e^{i \phi_{H}}$ and use the 
equivariant parameter $\left(b-b^{-1}\right)$, getting $-\sum_{m \geq 0} e^{i\left(b-b^{-1}\right) m}$. Putting everything together, and recalling that the multiplet transforms in a gauge representation $\mathcal{R}$, the contribution to the index of a chiral multiplet from the northern circle is:

$$
\text { ind } \operatorname{chiral}_{N}=-\sum_{w \in \mathcal{R}} \sum_{n \in \mathbb{Z}} e^{i b n} \sum_{m \geq 0} e^{i\left(b-b^{-1}\right) m} e^{-\frac{i}{2} Q R} e^{w\left(\hat{a}_{N}\right)}
$$

where $Q \equiv b+b^{-1}$ and $\hat{a}=-i\left(b A_{\varphi}+b^{-1} A \chi\right)-r \mathfrak{S}$.

At the southern circle, $\theta=\frac{\pi}{2}$, the Hopf fiber is parametrized by $\chi$ and $\mathcal{Q}^{2}$ acts freely on it with equivariant parameter $b^{-1}$, therefore the KK modes yield $\sum_{n \in \mathbb{Z}} e^{i b^{-1} n}$. The SUSY variation around $\theta=\frac{\pi}{2}$ is schematically $-D_{\tilde{\theta}}+\frac{i}{\tilde{\theta}} D_{\phi_{H}} \sim D_{\bar{z}}\left(\right.$ where $\left.\tilde{\theta}=\frac{\pi}{2}-\theta\right)$ if we identify $z=\tilde{\theta} e^{-i \phi_{H}}$. Again we expand in $t$ and use equivariant parameter $\left(b-b^{-1}\right)$, getting $\sum_{m \geq 1} e^{i\left(b-b^{-1}\right) m}$. Putting together:

$$
\text { ind } \operatorname{chiral}_{S}=\sum_{w \in \mathcal{R}} \sum_{n \in \mathbb{Z}} e^{i b^{-1} n} \sum_{m \geq 1} e^{i\left(b-b^{-1}\right) m} e^{-\frac{i}{2} Q R} e^{w\left(\hat{a}_{S}\right)} .
$$

The one-loop determinant is extracted with (C.2). We get the non-regulated expression

$$
Z_{1-\text { loop }}^{\text {chiral }} "=" \prod_{w \in \mathcal{R}} \prod_{n \in \mathbb{Z}} \prod_{m \geq 0} \frac{(m+1) b+n b^{-1}-\frac{Q}{2} R-i w\left(\hat{a}_{S}\right)}{n b-m b^{-1}-\frac{Q}{2} R-i w\left(\hat{a}_{N}\right)} .
$$

This is the expression in (2.50), after a rescaling by $\sqrt{\ell \tilde{\ell}}$ of both numerator and denominator. If $\hat{a}_{N} \neq \hat{a}_{S}$, this expression cannot be further simplified; the regulated expression could be written in terms of infinite $q$-Pochhammer factors. In our case $\hat{a}_{N}=\hat{a}_{S} \equiv \hat{a}$, thus we can simplify coincident factors and, neglecting overall signs, we get

$$
Z_{1-\text { loop }}^{\text {chiral }}=" \prod_{w \in \mathcal{R}} \prod_{m, n \geq 0} \frac{m b+n b^{-1}+\left(1-\frac{R}{2}\right) Q-i w(\hat{a})}{m b+n b^{-1}+\frac{R}{2} Q+i w(\hat{a})}=\prod_{w \in \mathcal{R}} s_{b}\left(\frac{i Q}{2}(1-R)+w(\hat{a})\right) .
$$

This is the expression in (2.51), and the last regulated expression was found in [12] in terms of the double sine function.

The one-loop determinant of the vector multiplet is computed in a similar way, observing that the relevant complex is the de Rham complex: the index of its complexification is just 1 , therefore we get $\frac{1}{2}$. At the northern and southern circles the indices are $\frac{1}{2} \sum_{n \in \mathbb{Z}} e^{i b n+\alpha\left(\hat{a}_{N}\right)}$ and $\frac{1}{2} \sum_{n \in \mathbb{Z}} e^{i b^{-1} n+\alpha\left(\hat{a}_{S}\right)}$ respectively, summed over the roots $\alpha$ of the gauge group. Extracting the eigenvalues and regularizing, we get

$$
Z_{1-\text { loop }}^{\mathrm{vec}}=\prod_{\alpha>0} 2 \sinh \left(\pi b^{-1} \alpha\left(\hat{a}_{N}\right)\right) 2 \sinh \left(\pi b \alpha\left(\hat{a}_{S}\right)\right)
$$

where the product is over the positive roots and the normalization is somewhat arbitrary.

The space $S^{\mathbf{2}} \times S^{\mathbf{1}}$. The square of the supercharge reads in this case

$$
\mathcal{Q}^{2}=-\mathcal{L}_{\partial_{\tau}}^{A}+\frac{i}{r} \mathcal{L}_{\partial_{\varphi}}^{A}-\cos \theta \sigma-\frac{1}{2 r} R+i \frac{\mathfrak{z} j}{2 \xi r} F_{j}
$$


It generates a free rotation along $S^{1}$ (of radius $2 \xi r$ ) with equivariant parameter -1 , thus resulting in the KK contribution $\sum_{n \in \mathbb{Z}} e^{-i \pi n / \xi r}$, and a rotation of the base $S^{2}$ with fixed points at $\theta=0$ and $\theta=\pi$.

At $\theta=0$ the SUSY variation of a chiral multiplet is of the form $D_{\theta}+\frac{i}{\theta} D_{\varphi} \sim D_{\bar{z}}$ if we identify $z=\theta e^{i \varphi}$. As above, the one-loop determinant of the chiral multiplet is then obtained from the index of the Dolbeault operator with inverted grading, which is $-\frac{1}{1-z}$. We expand in $t=e^{i \varphi}$ and use the equivariant parameter $\frac{i}{r}$, getting $-\sum_{k \geq 0} e^{-k / r}$. The total index at the north pole is thus:

$$
\text { ind } \operatorname{chiral}_{N}=-\sum_{w \in \mathcal{R}} \sum_{n \in \mathbb{Z}} e^{-\pi i n / \xi r} \sum_{k \geq 0} e^{-k / r} e^{-\frac{1}{2 r} R} e^{i \frac{\hat{z} j}{2 \xi r} F_{j}} e^{w\left(\hat{a}_{N}\right)}
$$

where $\hat{a}=i A_{\tau}+\frac{1}{r} A_{\varphi}-\cos \theta \sigma$. Similarly, at $\theta=\pi$ the SUSY variation is of the form $D_{\tilde{\theta}}+\frac{i}{\tilde{\theta}} D_{\varphi} \sim D_{\bar{z}}$ (where $\tilde{\theta}=\pi-\theta$ ) if we identify $z=\tilde{\theta} e^{i \varphi}$. Now we expand the index of the Dolbeault operator in $t^{-1}$ (since the orientation is opposite) and use the equivariant parameter $\frac{i}{r}$, getting $\sum_{k \geq 1} e^{k / r}$. The total index at the south pole is thus:

$$
\text { ind } \operatorname{chiral}_{S}=\sum_{w \in \mathcal{R}} \sum_{n \in \mathbb{Z}} e^{-\pi i n / \xi r} \sum_{k \geq 1} e^{k / r} e^{-\frac{1}{2 r} R} e^{i \frac{\hat{z}_{j}}{2 \xi r} F_{j}} e^{w\left(\hat{a}_{S}\right)} .
$$

The one-loop determinant is extracted with (C.2), obtaining the non-regulated expression:

$$
Z_{1-\text { loop }}^{\text {chiral } ~}=" \prod_{w \in \mathcal{R}} \prod_{n \in \mathbb{Z}} \prod_{k \geq 0} \frac{-\pi i n+(k+1) \xi-\frac{\xi}{2} R+\frac{i}{2} \sum_{j} \mathfrak{z}_{j} F_{j}+\xi r w\left(\hat{a}_{S}\right)}{-\pi i n-k \xi-\frac{\xi}{2} R+\frac{i}{2} \sum_{j} \mathfrak{z}_{j} F_{j}+\xi r w\left(\hat{a}_{N}\right)}
$$

For the vector multiplet, a computation exactly parallel to the one for $S_{b}^{3}$ gives

$$
\begin{aligned}
Z_{1-\text { loop }}^{\mathrm{vec}} " & =" \prod_{\alpha \in \mathfrak{g}} \prod_{n \in \mathbb{Z}}\left(\alpha\left(\hat{a}_{N}\right)+\frac{2 \pi i n}{2 \xi r}\right)^{1 / 2}\left(\alpha\left(\hat{a}_{S}\right)+\frac{2 \pi i n}{2 \xi r}\right)^{1 / 2} \\
& =\prod_{\alpha>0} 2 \sinh \left(\xi r \alpha\left(\hat{a}_{N}\right)\right) 2 \sinh \left(-\xi r \alpha\left(\hat{a}_{S}\right)\right)
\end{aligned}
$$

where the product runs over the positive roots.

Open Access. This article is distributed under the terms of the Creative Commons Attribution License (CC-BY 4.0), which permits any use, distribution and reproduction in any medium, provided the original author(s) and source are credited.

\section{References}

[1] E. Witten, Topological quantum field theory, Commun. Math. Phys. 117 (1988) 353 [INSPIRE].

[2] E. Witten, Mirror manifolds and topological field theory, hep-th/9112056 [INSPIRE].

[3] V. Pestun, Localization of gauge theory on a four-sphere and supersymmetric Wilson loops, Commun. Math. Phys. 313 (2012) 71 [arXiv:0712.2824] [INSPIRE]. 
[4] F. Benini and S. Cremonesi, Partition functions of $\mathcal{N}=(2,2)$ gauge theories on $S^{2}$ and vortices, arXiv:1206.2356 [INSPIRE].

[5] N. Doroud, J. Gomis, B. Le Floch and S. Lee, Exact Results in D $=2$ Supersymmetric Gauge Theories, JHEP 05 (2013) 093 [arXiv:1206.2606] [INSPIRE].

[6] A. Gadde and S. Gukov, 2d index and surface operators, JHEP 03 (2014) 080 [arXiv: 1305.0266] [INSPIRE].

[7] F. Benini, R. Eager, K. Hori and Y. Tachikawa, Elliptic genera of two-dimensional $N=2$ gauge theories with rank-one gauge groups, Lett. Math. Phys. 104 (2014) 465 [arXiv: 1305.0533] [INSPIRE].

[8] F. Benini, R. Eager, K. Hori and Y. Tachikawa, Elliptic genera of $2 d N=2$ gauge theories, arXiv:1308.4896 [INSPIRE].

[9] A. Kapustin, B. Willett and I. Yaakov, Exact Results for Wilson Loops in Superconformal Chern-Simons Theories with Matter, JHEP 03 (2010) 089 [arXiv:0909.4559] [INSPIRE].

[10] D.L. Jafferis, The exact superconformal R-symmetry extremizes Z, JHEP 05 (2012) 159 [arXiv: 1012.3210] [INSPIRE].

[11] N. Hama, K. Hosomichi and S. Lee, Notes on SUSY gauge theories on three-sphere, JHEP 03 (2011) 127 [arXiv: 1012.3512] [INSPIRE].

[12] N. Hama, K. Hosomichi and S. Lee, SUSY gauge theories on squashed three-spheres, JHEP 05 (2011) 014 [arXiv: 1102.4716] [INSPIRE].

[13] Y. Imamura and D. Yokoyama, $N=2$ supersymmetric theories on squashed three-sphere, Phys. Rev. D 85 (2012) 025015 [arXiv: 1109.4734] [INSPIRE].

[14] L.F. Alday, D. Martelli, P. Richmond and J. Sparks, Localization on Three-Manifolds, arXiv:1307.6848 [INSPIRE].

[15] S. Kim, The Complete superconformal index for $N=6$ Chern-Simons theory, Nucl. Phys. B 821 (2009) 241 [Erratum ibid. B 864 (2012) 884] [arXiv:0903.4172] [INSPIRE].

[16] Y. Imamura and S. Yokoyama, Index for three dimensional superconformal field theories with general R-charge assignments, JHEP 04 (2011) 007 [arXiv: 1101.0557] [INSPIRE].

[17] N. Hama and K. Hosomichi, Seiberg-Witten Theories on Ellipsoids, JHEP 09 (2012) 033 [Addendum ibid. 1210 (2012) 051] [arXiv:1206.6359] [INSPIRE].

[18] C. Closset and I. Shamir, The $\mathcal{N}=1$ Chiral Multiplet on $T^{2} \times S^{2}$ and Supersymmetric Localization, JHEP 03 (2014) 040 [arXiv: 1311.2430] [INSPIRE].

[19] J. Källén and M. Zabzine, Twisted supersymmetric 5D Yang-Mills theory and contact geometry, JHEP 05 (2012) 125 [arXiv:1202.1956] [INSPIRE].

[20] K. Hosomichi, R.-K. Seong and S. Terashima, Supersymmetric Gauge Theories on the Five-Sphere, Nucl. Phys. B 865 (2012) 376 [arXiv:1203.0371] [inSPIRE].

[21] H.-C. Kim and S. Kim, M5-branes from gauge theories on the 5-sphere, JHEP 05 (2013) 144 [arXiv: 1206.6339] [INSPIRE].

[22] Y. Imamura, Supersymmetric theories on squashed five-sphere, PTEP 2013 (2013) $013 \mathrm{~B} 04$ [arXiv: 1209.0561] [INSPIRE]. 
[23] S. Pasquetti, Factorisation of $\mathcal{N}=2$ Theories on the Squashed 3-Sphere, JHEP 04 (2012) 120 [arXiv:1111.6905] [INSPIRE].

[24] T. Dimofte, D. Gaiotto and S. Gukov, Gauge Theories Labelled by Three-Manifolds, Commun. Math. Phys. 325 (2014) 367 [arXiv:1108.4389] [InSPIRE].

[25] S. Shadchin, On F-term contribution to effective action, JHEP 08 (2007) 052 [hep-th/0611278] [INSPIRE].

[26] N.A. Nekrasov, Seiberg-Witten prepotential from instanton counting, Adv. Theor. Math. Phys. 7 (2004) 831 [hep-th/0206161] [INSPIRE].

[27] S. Cecotti and C. Vafa, Topological antitopological fusion, Nucl. Phys. B 367 (1991) 359 [INSPIRE].

[28] C. Beem, T. Dimofte and S. Pasquetti, Holomorphic blocks in three dimensions, arXiv:1211.1986 [INSPIRE].

[29] M. Taki, Holomorphic blocks for $3 d$ non-abelian partition functions, arXiv:1303.5915 [INSPIRE].

[30] C. Krattenthaler, V.P. Spiridonov and G.S. Vartanov, Superconformal indices of three-dimensional theories related by mirror symmetry, JHEP 06 (2011) 008 [arXiv:1103.4075] [INSPIRE].

[31] C. Hwang, H.-C. Kim and J. Park, Factorization of the 3d superconformal index, arXiv: 1211.6023 [INSPIRE].

[32] C. Closset, T.T. Dumitrescu, G. Festuccia and Z. Komargodski, The geometry of supersymmetric partition functions, JHEP 01 (2014) 124 [arXiv:1309.5876] [INSPIRE].

[33] S. Cecotti, D. Gaiotto and C. Vafa, $t t^{*}$ Geometry in 3 and 4 Dimensions, arXiv:1312.1008 [INSPIRE].

[34] C. Klare, A. Tomasiello and A. Zaffaroni, Supersymmetry on Curved Spaces and Holography, JHEP 08 (2012) 061 [arXiv: 1205.1062] [INSPIRE].

[35] C. Closset, T.T. Dumitrescu, G. Festuccia and Z. Komargodski, Supersymmetric Field Theories on Three-Manifolds, JHEP 05 (2013) 017 [arXiv:1212.3388] [inSPIRE].

[36] N. Nekrasov and A. Okounkov, Seiberg-Witten theory and random partitions, hep-th/0306238 [INSPIRE].

[37] F. Benini, T. Nishioka and M. Yamazaki, 4d Index to 3d Index and $2 d$ TQFT, Phys. Rev. D 86 (2012) 065015 [arXiv:1109.0283] [INSPIRE].

[38] L.F. Alday, M. Fluder and J. Sparks, The large-N limit of M2-branes on Lens spaces, JHEP 10 (2012) 057 [arXiv: 1204.1280] [INSPIRE].

[39] C. Romelsberger, Counting chiral primaries in $N=1, D=4$ superconformal field theories, Nucl. Phys. B 747 (2006) 329 [hep-th/0510060] [INSPIRE].

[40] A. Gadde, E. Pomoni, L. Rastelli and S.S. Razamat, S-duality and 2d Topological QFT, JHEP 03 (2010) 032 [arXiv:0910.2225] [INSPIRE].

[41] S.S. Razamat and B. Willett, Global Properties of Supersymmetric Theories and the Lens Space, arXiv:1307.4381 [INSPIRE].

[42] M. Fujitsuka, M. Honda and Y. Yoshida, Higgs branch localization of $3 d N=2$ theories, arXiv:1312.3627 [INSPIRE]. 
[43] D. Martelli, A. Passias and J. Sparks, The gravity dual of supersymmetric gauge theories on a squashed three-sphere, Nucl. Phys. B 864 (2012) 840 [arXiv:1110.6400] [InSPIRE].

[44] E. Witten, Two-dimensional gauge theories revisited, J. Geom. Phys. 9 (1992) 303 [hep-th/9204083] [INSPIRE].

[45] M.F. Atiyah, Elliptic operators and compact groups, in Lecture Notes in Mathematics. Vol. 401, Springer-Verlag, Berlin Germany (1974).

[46] N. Drukker, T. Okuda and F. Passerini, Exact results for vortex loop operators in $3 d$ supersymmetric theories, arXiv:1211.3409 [INSPIRE].

[47] J. Gomis, T. Okuda and V. Pestun, Exact results for 't Hooft loops in gauge theories on $S^{4}$, JHEP 05 (2012) 141 [arXiv:1105.2568] [INSPIRE].

[48] A.G. Bytsko and J. Teschner, Quantization of models with non-compact quantum group symmetry: Modular XXZ magnet and lattice sinh-Gordon model,

J. Phys. A 39 (2006) 12927 [hep-th/0602093] [INSPIRE].

[49] F. Benini, C. Closset and S. Cremonesi, Comments on 3d Seiberg-like dualities, JHEP 10 (2011) 075 [arXiv: 1108.5373] [INSPIRE].

[50] P. Goddard, J. Nuyts and D.I. Olive, Gauge Theories and Magnetic Charge, Nucl. Phys. B 125 (1977) 1 [INSPIRE].

[51] O. Aharony, A. Hanany, K.A. Intriligator, N. Seiberg and M.J. Strassler, Aspects of $N=2$ supersymmetric gauge theories in three-dimensions, Nucl. Phys. B 499 (1997) 67 [hep-th/9703110] [INSPIRE].

[52] H.-C. Kim, J. Kim, S. Kim and K. Lee, Vortices and 3 dimensional dualities, arXiv:1204.3895 [INSPIRE].

[53] H.-Y. Chen, H.-Y. Chen and J.-K. Ho, Connecting Mirror Symmetry in $3 d$ and $2 d$ via Localization, arXiv:1312.2361 [INSPIRE].

[54] J. Bhattacharya, S. Bhattacharyya, S. Minwalla and S. Raju, Indices for Superconformal Field Theories in 3,5 and 6 Dimensions, JHEP 02 (2008) 064 [arXiv:0801.1435] [INSPIRE].

[55] A. Kapustin and B. Willett, Generalized Superconformal Index for Three Dimensional Field Theories, arXiv:1106.2484 [INSPIRE].

[56] O. Aharony, S.S. Razamat, N. Seiberg and B. Willett, 3d dualities from $4 d$ dualities, JHEP 07 (2013) 149 [arXiv:1305.3924] [INSPIRE].

[57] T.T. Wu and C.N. Yang, Dirac Monopole Without Strings: Monopole Harmonics, Nucl. Phys. B 107 (1976) 365 [INSPIRE].

[58] F. Benini, Y. Tachikawa and D. Xie, Mirrors of 3d Sicilian theories, JHEP 09 (2010) 063 [arXiv: 1007.0992] [INSPIRE].

[59] D. Gaiotto, $N=2$ dualities, JHEP 08 (2012) 034 [arXiv:0904.2715] [INSPIRE].

[60] D. Gaiotto, G.W. Moore and A. Neitzke, Wall-crossing, Hitchin Systems and the WKB Approximation, arXiv:0907.3987 [INSPIRE].

[61] D. Gaiotto and D.L. Jafferis, Notes on adding D6 branes wrapping $R p^{3}$ in $A d S_{4} \times C P^{3}$, JHEP 11 (2012) 015 [arXiv:0903.2175] [INSPIRE].

[62] F. Benini, C. Closset and S. Cremonesi, Chiral flavors and M2-branes at toric CY4 singularities, JHEP 02 (2010) 036 [arXiv:0911.4127] [INSPIRE]. 
[63] D.L. Jafferis, Quantum corrections to $\mathcal{N}=2$ Chern-Simons theories with flavor and their $A d S_{4}$ duals, JHEP 08 (2013) 046 [arXiv:0911.4324] [INSPIRE].

[64] F. Benini, C. Closset and S. Cremonesi, Quantum moduli space of Chern-Simons quivers, wrapped D6-branes and AdS4/CFT3, JHEP 09 (2011) 005 [arXiv: 1105.2299] [INSPIRE].

[65] Y. Kosmann, Dérivées de Lie des spineurs, Ann. Mat. Pura Appl. 91 (1971) 317.

[66] M. Godina and P. Matteucci, Reductive G structures and Lie derivatives, J. Geom. Phys. 47 (2003) 66 [math/0201235] [INSPIRE]. 